\title{
NUMERICAL ANALYSIS OF THE PLANEWAVE DISCRETIZATION OF SOME ORBITAL-FREE AND KOHN-SHAM MODELS
}

\author{
ERIC CANCÈs ${ }^{1}$, RACHIDA ChAKIR ${ }^{2,3}$ AND YVON MADAY ${ }^{2,3,4}$
}

\begin{abstract}
In this article, we provide a priori error estimates for the spectral and pseudospectral Fourier (also called planewave) discretizations of the periodic Thomas-Fermi-von Weizsäcker (TFW) model and for the spectral discretization of the periodic Kohn-Sham model, within the local density approximation (LDA). These models allow to compute approximations of the electronic ground state energy and density of molecular systems in the condensed phase. The TFW model is strictly convex with respect to the electronic density, and allows for a comprehensive analysis. This is not the case for the Kohn-Sham LDA model, for which the uniqueness of the ground state electronic density is not guaranteed. We prove that, for any local minimizer $\Phi^{0}$ of the Kohn-Sham LDA model, and under a coercivity assumption ensuring the local uniqueness of this minimizer up to unitary transform, the discretized Kohn-Sham LDA problem has a minimizer in the vicinity of $\Phi^{0}$ for large enough energy cut-offs, and that this minimizer is unique up to unitary transform. We then derive optimal a priori error estimates for the spectral discretization method.
\end{abstract}

Mathematics Subject Classification. 65N25, 65N35, 65T99, 35P30, 35Q40, 81Q05.

Received April 8, 2010. Revised March 22, 2011.

Published online October 24, 2011.

\section{INTRODUCTION}

First-principle molecular simulation allows to better understand, or to predict, the properties of matter from the fundamental laws of quantum mechanics. It is a major tool in chemistry, condensed matter physics, and materials science, used on a daily basis by hundreds of research groups in academy and industry. It is also becoming a fruitful approach in molecular biology and nanotechnologies.

In this approach, matter is described as an assembly of nuclei and electrons. At this scale, the equation that rules the interactions between these constitutive elements is the $N$-body Schrödinger equation. It can be considered (except in few special cases notably those involving relativistic phenomena or nuclear reactions) as a universal model for at least three reasons. First, it contains all the physical information of the system under consideration, so that any of the properties of this system can be deduced in theory from the Schrödinger

\footnotetext{
Keywords and phrases. Electronic structure calculation, density functional theory, Thomas-Fermi-von Weizsäcker model, Kohn-Sham model, nonlinear eigenvalue problem, spectral methods.

${ }^{1}$ Université Paris-Est, CERMICS, Project-team Micmac, INRIA-École des Ponts, 6 \& 8 avenue Blaise Pascal, 77455 Marne-laVallée Cedex 2, France. cances@cermics.enpc.fr

2 UPMC Univ. Paris 06, UMR 7598 LJLL, 75005 Paris, France

3 CNRS, UMR 7598 LJLL, 75005 Paris, France

${ }^{4}$ Division of Applied Mathematics, 182 George Street, Brown University, Providence, RI 02912, USA
} 
equation associated to it. Second, the Schrödinger equation does not involve any empirical parameter, except some fundamental constants of physics (the Planck constant, the mass and charge of the electron, ... ); it can thus be written for any kind of molecular system provided its chemical composition, in terms of natures of nuclei and number of electrons, is known. Third, this model enjoys remarkable predictive capabilities, as confirmed by comparisons with a large amount of experimental data of various types.

Of course, the $N$-body time-dependent Schrödinger equation, which is an evolution partial differential equation in space dimension $3 N$, where $N$ is the number of particles (nuclei and electrons) in the system, cannot be solved by brute force numerical methods when $N$ exceeds three or four. In order to deal with larger systems, approximations have to be resorted to. The Born-Oppenheimer approximation [4], based on the fact that nuclei are thousands of times heavier than electrons, allows to decouple the nuclear and electronic dynamics, and to consider that, in most cases, nuclei behave as point-like classical particles, and electrons are in their ground state. Several methods for computing approximations of electronic ground states have then been proposed, which can be classified in three groups:

- wavefunction methods, among which the Hartree-Fock and multiconfiguration self-consistent-field (MCSCF) models (see [8] for a mathematical introduction);

- methods issued from the density functional theory (DFT);

- quantum Monte Carlo methods [24,25].

DFT currently is the most popular approach for it offers the best compromize between accuracy and computation cost. The models originating from DFT can be classified into two categories: the orbital-free models and the Kohn-Sham models. The Thomas-Fermi-von Weizsäcker (TFW) model falls into the first category. It is not very much used in practice, but is interesting from both a mathematical viewpoint $[2,12,27]$ and a numerical viewpoint [35]. It indeed serves as a toy model for the analysis of the more complex electronic structure models routinely used by physicists and chemists, as well as for the development of new numerical methods [17,31]. At the other extremity of the spectrum, the Kohn-Sham models [15,21] are among the most widely used models in physics and chemistry, but are much more difficult to deal with.

Throughout this article, we adopt the system of atomic units for which $\hbar=1$ (reduced Planck constant), $m_{e}=1$ (mass of the electron), $e=1$ (elementary charge), $4 \pi \epsilon_{0}=1$ ( $\epsilon_{0}$ being the dielectric permittivity of the vacuum). In this system of units, the charge of the electron is -1 and the charges of nuclei are positive integers.

Let us first consider an isolated molecular system in vacuo, consisting of $M$ nuclei of charges $\left(z_{1}, \ldots, z_{M}\right) \in$ $(\mathbb{N} \backslash\{0\})^{M}$ located at the positions $\left(R_{1}, \ldots, R_{M}\right) \in\left(\mathbb{R}^{3}\right)^{M}$ of the physical space, and of $\mathcal{N}$ electrons. The electrostatic potential generated by the nuclei and felt by the electrons is

$$
V^{\mathrm{nuc}}(x)=-\sum_{k=1}^{M} \frac{z_{k}}{\left|x-R_{k}\right|}
$$

In the TFW model, as well as in any orbital-free model, the ground state electronic density of the system is obtained by minimizing an explicit functional of the density. For the system under consideration, this model reads $[27]$

$$
\inf \left\{\mathcal{E}_{0}^{\mathrm{TFW}}(\rho), \rho \geq 0, \sqrt{\rho} \in H^{1}\left(\mathbb{R}^{3}\right), \int_{\mathbb{R}^{3}} \rho=\mathcal{N}\right\},
$$

where $\mathcal{E}_{0}^{\mathrm{TFW}}(\rho)$ is the TFW energy functional defined as

$$
\mathcal{E}_{0}^{\mathrm{TFW}}(\rho):=\frac{C_{\mathrm{W}}}{2} \int_{\mathbb{R}^{3}}|\nabla \sqrt{\rho}|^{2}+C_{\mathrm{TF}} \int_{\mathbb{R}^{3}} \rho^{5 / 3}+\int_{\mathbb{R}^{3}} \rho V^{\mathrm{nuc}}+\frac{1}{2} D(\rho, \rho),
$$

and where the bilinear form $D(\cdot, \cdot)$ is the Coulomb energy functional in vacuo:

$$
D\left(\rho, \rho^{\prime}\right):=\int_{\mathbb{R}^{3}} \int_{\mathbb{R}^{3}} \frac{\rho(x) \rho^{\prime}(y)}{|x-y|} \mathrm{d} x \mathrm{~d} y=4 \pi \int_{\mathbb{R}^{3}}|k|^{-2} \widehat{\rho}(k)^{*} \widehat{\rho}^{\prime}(k) \mathrm{d} k,
$$


$\widehat{f}$ denoting the Fourier transform of $f$ (normalized in such a way that the Fourier transform is the isometry of $\left.L^{2}\left(\mathbb{R}^{3}\right)\right)$. Here and in the sequel $a^{*}$ denotes the complex conjugate of the complex number $a$. The first two terms of the TFW energy functional model the kinetic energy of the electrons; $C_{\mathrm{W}}$ is a positive real number $\left(C_{\mathrm{W}}=1\right.$, $1 / 5$ or $1 / 9$ depending on the context [15]) and $C_{\mathrm{TF}}$ is the Thomas-Fermi constant $\left(C_{\mathrm{TF}}=\frac{10}{3}\left(3 \pi^{2}\right)^{2 / 3}\right)$. The third and fourth terms respectively model the interactions between nuclei and electrons, and the interactions between electrons.

In the Kohn-Sham model, the electronic state of the closed-shell system with an even number $\mathcal{N}=2 N$ of electrons is described by $N$ Kohn-Sham orbitals $\Phi=\left(\phi_{1}, \ldots, \phi_{N}\right)^{T} \in\left(H^{1}\left(\mathbb{R}^{3}\right)\right)^{N}$ satisfying the orthonormality conditions

and the associated electronic density

$$
\int_{\mathbb{R}^{3}} \phi_{i} \phi_{j}=\delta_{i j}
$$

$$
\rho_{\Phi}(x):=2 \sum_{i=1}^{N}\left|\phi_{i}(x)\right|^{2} .
$$

The factor 2 in the above expression accounts for the spin. In closed-shell systems, each Kohn-Sham orbital is indeed occupied by two electrons, one with spin up and one with spin down. The Kohn-Sham ground state is obtained by solving the minimization problem

$$
\inf \left\{\mathcal{E}_{0}^{\mathrm{KS}}(\Phi), \Phi=\left(\phi_{1}, \ldots, \phi_{N}\right)^{T} \in\left(H^{1}\left(\mathbb{R}^{3}\right)\right)^{N}, \int_{\mathbb{R}^{3}} \phi_{i} \phi_{j}=\delta_{i j}\right\}
$$

where the Kohn-Sham energy functional reads

$$
\mathcal{E}_{0}^{\mathrm{KS}}(\Phi):=\sum_{i=1}^{N} \int_{\mathbb{R}^{3}}\left|\nabla \phi_{i}\right|^{2}+\int_{\mathbb{R}^{3}} V^{\mathrm{nuc}} \rho_{\Phi}+\frac{1}{2} D\left(\rho_{\Phi}, \rho_{\Phi}\right)+E_{\mathrm{xc}}\left(\rho_{\Phi}\right)
$$

The first term models the kinetic energy, the second term the interactions between nuclei and electrons, and the third term the interaction between electrons. The fourth term, called the exchange-correlation functional, is a correction term, which is essential to describe quantitatively, and sometimes even qualitatively, the physics and chemistry of the system. The exchange-correlation functional collects the errors made in the approximations of the kinetic energy and of the interactions between electrons by respectively the first and third terms of the Kohn-Sham functional. It follows from the Hohenberg-Kohn theorem [20,26,28,33], that there exists an exact exchange-correlation functional, that is a functional of the electronic density $\rho$ for which solving (1.4) provides the ground state electronic energy and density of the $N$-body electronic Schrödinger equation. Unfortunately, no mathematical expression of the exchange-correlation functional amenable to numerical simulations is known. It therefore has to be approximated in practice. The local density approximation (LDA) consists in approximating the exchange-correlation functional by

$$
\int_{\mathbb{R}^{3}} e_{\mathrm{xc}}^{\mathrm{LDA}}(\rho(x)) \mathrm{d} x
$$

where $e_{\mathrm{xc}}^{\mathrm{LDA}}(\bar{\rho})$ is an approximation of the exchange-correlation energy per unit volume in a uniform electron gas with density $\bar{\rho}$. The resulting Kohn-Sham LDA model is well understood from a mathematical viewpoint $[1,23]$. On the other hand, the existence of minimizers for Kohn-Sham models based on more refined approximations of the exchange-correlation functional, such as generalized gradient approximations [1] or exact local exchange potentials [10], still is an open problem.

In the sequel, we will focus on the periodic versions of the TFW and Kohn-Sham LDA models. In the periodic setting, the simulation domain, sometimes referred to as the supercell, is no longer the whole space $\mathbb{R}^{3}$, as in (1.2)-(1.4); it is the unit cell $\Gamma$ of some periodic lattice of $\mathbb{R}^{3}$. In the periodic TFW model, periodic boundary conditions (PBC) are imposed to the density; in the periodic Kohn-Sham framework, they are imposed to the Kohn-Sham orbitals (Born-von Karman PBC). Imposing PBC at the boundary of the simulation cell is 
the standard method to compute condensed phase properties with a limited number of atoms in the simulation cell, hence at a moderate computational cost.

In most applications, the periodic TFW and Kohn-Sham models are discretized in Fourier modes, more commonly referred to as planewave basis sets in the physics and chemistry literature. This is the reason why we focus on this particular discretization method in the present work. The TFW ground state density corresponding to the nuclear potential (1.1) is known to have cusps at the nuclear positions $R_{k}$. These singularities reduces the efficiency of the planewave discretization method. In practice, the singular nuclear potential $V^{\text {nuc }}$ defined by (1.1) is usually replaced with a smoother potential $V^{\text {ion}}$; this amounts to replacing point nuclei with smeared nuclei. We will see in Section 3 that, not surprisingly, the smoother the potential, the faster the convergence of the planewave approximation to the exact solution of (1.2). In the Kohn-Sham setting, this issue is addressed in a more refined way [32], for it is also considered that core electrons are not affected by the chemical environment. The Kohn-Sham orbitals of the core electrons surrounding each nucleus (for instance the two $1 s$ electrons of the nuclei of the second row of the periodic table) are frozen to their ground states in the isolated atom. The KohnSham orbitals of the valence electrons (i.e. of the electrons which are not core electrons and are therefore affected by the chemical environment) are replaced with pseudo-orbitals, which coincide with the valence Kohn-Sham orbitals out of a so-called core region surrounding each nucleus, and are smoother than the valence Kohn-Sham orbitals inside the core region. The resulting model is similar to (1.4), but presents some differences: (i) $N$ now denotes the number of valence electron pairs, (ii) $\Phi$ now denotes the set of the pseudo-orbitals of the valence electrons, and (iii) the nuclear potential $V^{\text {nuc }}$ is replaced by a pseudopotential modeling the Coulomb interaction between the valence electrons on the one hand, and the nuclei and the core electrons on the other hand. The pseudopotential consists of two terms: a local component $V_{\text {local }}$ (whose associated operator is the multiplication by the function $V_{\text {local }}$ ) and a nonlocal component (an operator whose expression is given in Section 4). As a consequence, the second term in the Kohn-Sham energy functional (1.5) is replaced by

$$
\int_{\Gamma} \rho_{\Phi} V_{\text {local }}+2 \sum_{i=1}^{N}\left\langle\phi_{i}\left|V_{\mathrm{nl}}\right| \phi_{i}\right\rangle .
$$

The pseudopotential approximation has two main advantages: first, it allows to deal with heavy nuclei (for which core electrons are relativistic) within a non-relativistic framework, and, second, it reduces the computational cost by reducing the number $N$ of orbitals to be computed and by regularizing these orbitals (hence increasing the rate of convergence of the planewave approximation). The pseudopotential appoximation gives satisfactory results in most cases, but sometimes fails. A mathematical analysis of the pseudopotential approximation is still lacking.

The purpose of this article is to provide an analysis of the Fourier spectral and pseudospectral discretizations of the periodic TFW and Kohn-Sham LDA models, following our first contribution [6] dealing with simpler nonlinear eigenvalue problems. As far as we know, our results are the first ones presenting the optimal convergence rate for the ground state energy and eigenpairs, both for the TFW type problems where some papers already existed, and for the Kohn-Sham problem where no numerical analysis in terms of convergence was available. Previous contributions in the numerical analysis of electronic structure models are actually very few. In [35], the convergence of the ground state energy and eigenpair is established for the Galerkin discretization of a convex TFW model, but no optimal rate of convergence is provided. In [22], the authors have considered the Thomas-Fermi-Dirac-von Weizsäcker model, that is a non convex model entering in the category of orbital-free DFT models. Under an hypothesis of ellipticity of the second order derivative of the Lagrangian associated with the minimization problem, they prove that the discrete problem, based on a $\mathbb{P}_{1}$-Lagrange finite element approximation has, locally, a unique discrete solution that converges at the optimal rate in the energy norm. The convergence of the eigenvalue is also obtained, but is not optimal.

More recently, Zhou et al. have analyzed a non-convex orbital-free model [14]; again with an assumption of local inversibility in the vicinity of the ground states, the convergence of the minimizers of the discrete problem to the set of the minimizers of the continuous problem is established, but no convergence rate is actually proven. 
All the results mentioned above deal with a priori analysis. The results about a posteriori error analysis are even more seldom. We refer to $[13,29]$ for the available results in this direction and improvements of the basic approximation by either postprocessing, or thanks to adaptivity.

This article is organized as follows. In Section 2, we briefly introduce the functional setting used in the formulation and the analysis of the planewave discretization of periodic orbital-free and Kohn-Sham models. In Section 3, we provide a priori error estimates for the planewave discretization of the periodic TFW model, including numerical integration. In Section 4, we deal with the periodic Kohn-Sham LDA model.

\section{BAsic Fourier anAlysis For PlanewaVe DisCREtization methods}

Throughout this article, we denote by $\Gamma$ the simulation cell, by $\mathcal{R}$ the periodic lattice, and by $\mathcal{R}^{*}$ the dual lattice. For simplicity, we assume that $\Gamma=[0, L)^{3}(L>0)$, in which case $\mathcal{R}$ is the cubic lattice $L \mathbb{Z}^{3}$, and $\mathcal{R}^{*}=\frac{2 \pi}{L} \mathbb{Z}^{3}$. Our arguments can be easily extended to the general case. For $k \in \mathcal{R}^{*}$, we denote by $e_{k}(x)=|\Gamma|^{-1 / 2} \mathrm{e}^{\mathrm{i} k \cdot x}$ the planewave with wavevector $k$. The family $\left(e_{k}\right)_{k \in \mathcal{R}^{*}}$ forms an orthonormal basis of

$$
L_{\#}^{2}(\Gamma, \mathbb{C}):=\left\{u \in L_{\text {loc }}^{2}\left(\mathbb{R}^{3}, \mathbb{C}\right) \mid u \mathcal{R} \text {-periodic }\right\}
$$

and for all $u \in L_{\#}^{2}(\Gamma, \mathbb{C})$,

$$
u(x)=\sum_{k \in \mathcal{R}^{*}} \widehat{u}_{k} e_{k}(x) \quad \text { with } \quad \widehat{u}_{k}=\left(e_{k}, u\right)_{L_{\#}^{2}}=|\Gamma|^{-1 / 2} \int_{\Gamma} u(x) \mathrm{e}^{-\mathrm{i} k \cdot x} \mathrm{~d} x .
$$

In our analysis, we will mainly consider real valued functions. We therefore introduce the Sobolev spaces of real valued $\mathcal{R}$-periodic functions

$$
H_{\#}^{s}(\Gamma):=\left\{u(x)=\left.\sum_{k \in \mathcal{R}^{*}} \widehat{u}_{k} e_{k}(x)\left|\sum_{k \in \mathcal{R}^{*}}\left(1+|k|^{2}\right)^{s}\right| \widehat{u}_{k}\right|^{2}<\infty \text { and } \forall k, \widehat{u}_{-k}=\widehat{u}_{k}^{*}\right\},
$$

$s \in \mathbb{R}$, endowed with the inner products

$$
(u, v)_{H_{\#}^{s}}=\sum_{k \in \mathcal{R}^{*}}\left(1+|k|^{2}\right)^{s} \widehat{u}_{k}^{*} \widehat{v}_{k} .
$$

For $N_{c} \in \mathbb{N}$, we denote by

$$
V_{N_{c}}=\left\{\sum_{k \in \mathcal{R}^{*}|| k \mid \leq \frac{2 \pi}{L} N_{c}} c_{k} e_{k} \mid \forall k, c_{-k}=c_{k}^{*}\right\}
$$

(the constraints $c_{-k}=c_{k}^{*}$ imply that the functions of $V_{N_{c}}$ are real valued). The norm $|\cdot|$ used in the definition of $V_{N_{c}}$ is the Euclidian norm. The plane wave $e_{k}$ belongs to $V_{N_{c}}$ if and only if its kinetic energy $\frac{1}{2}|k|^{2}$ is smaller than the energy cut-off $E_{\mathrm{c}}=\frac{2 \pi^{2}}{L^{2}} N_{c}^{2}$. For all $s \in \mathbb{R}$, and each $v \in H_{\#}^{s}(\Gamma)$, the best approximation of $v$ in $V_{N_{c}}$ for any $H_{\#}^{r}$-norm, $r \leq s$, is

$$
\Pi_{N_{c}} v=\sum_{k \in \mathcal{R}^{*}|| k \mid \leq \frac{2 \pi}{L} N_{c}} \widehat{v}_{k} e_{k}
$$


The more regular $v$ (the regularity being measured in terms of the Sobolev norms $H^{r}$ ), the faster the convergence of this truncated series to $v$ : for all real numbers $r$ and $s$ with $r \leq s$, we have for each $v \in H_{\#}^{s}(\Gamma)$,

$$
\begin{aligned}
\left\|v-\Pi_{N_{c}} v\right\|_{H_{\#}^{r}}=\min _{v_{N_{c}} \in V_{N_{c}}}\left\|v-v_{N_{c}}\right\|_{H_{\#}^{r}} & \leq\left(\frac{L}{2 \pi}\right)^{s-r} N_{c}^{-(s-r)}\left\|v-\Pi_{N_{c}} v\right\|_{H_{\#}^{s}} \\
& \leq\left(\frac{L}{2 \pi}\right)^{s-r} N_{c}^{-(s-r)}\|v\|_{H_{\#}^{s}} .
\end{aligned}
$$

For $N_{g} \in \mathbb{N} \backslash\{0\}$, we denote by $\widehat{\phi}^{\mathrm{FFT}, N_{g}}$ the discrete Fourier transform on the cartesian grid $\mathcal{G}_{N_{g}}:=\frac{L}{N_{g}} \mathbb{Z}^{3}$ of the function $\phi \in C_{\#}^{0}(\Gamma, \mathbb{C})$, where

$$
C_{\#}^{0}(\Gamma, \mathbb{C}):=\left\{u \in C^{0}\left(\mathbb{R}^{3}, \mathbb{C}\right) \mid u \mathcal{R} \text {-periodic }\right\} .
$$

Recall that if $\phi=\sum_{k \in \mathcal{R}^{*}} \widehat{\phi}_{k} e_{k} \in C_{\#}^{0}(\Gamma, \mathbb{C})$, then the discrete Fourier transform of $\phi$ is the $N_{g} \mathcal{R}^{*}$-periodic sequence $\widehat{\phi}^{\mathrm{FFT}, N_{g}}=\left(\widehat{\phi}_{k}^{\mathrm{FFT}, N_{g}}\right)_{k \in \mathcal{R}^{*}}$, where

$$
\widehat{\phi}_{k}^{\mathrm{FFT}, N_{g}}=\frac{1}{N_{g}^{3}} \sum_{x \in \mathcal{G}_{N_{g}} \cap \Gamma} \phi(x) \mathrm{e}^{-\mathrm{i} k \cdot x}=|\Gamma|^{-1 / 2} \sum_{K \in \mathcal{R}^{*}} \widehat{\phi}_{k+N_{g} K} .
$$

We now introduce the subspaces

$$
W_{N_{g}}^{1 \mathrm{D}}=\mid \begin{array}{ll}
\operatorname{Span}\left\{\mathrm{e}^{\mathrm{i} l y}\left|l \in \frac{2 \pi}{L} \mathbb{Z},\right| l \mid \leq \frac{2 \pi}{L}\left(\frac{N_{g}-1}{2}\right)\right\} & \left(N_{g} \text { odd }\right), \\
\operatorname{Span}\left\{\mathrm{e}^{\mathrm{i} l y}\left|l \in \frac{2 \pi}{L} \mathbb{Z},\right| l \mid \leq \frac{2 \pi}{L}\left(\frac{N_{g}}{2}\right)\right\} \oplus \mathbb{C}\left(\mathrm{e}^{\mathrm{i} \pi N_{g} y / L}+\mathrm{e}^{-\mathrm{i} \pi N_{g} y / L}\right) & \left(N_{g} \text { even }\right),
\end{array}
$$

$\left(W_{N_{g}}^{1 \mathrm{D}} \in C_{\#}^{\infty}([0, L), \mathbb{C})\right.$ and $\left.\operatorname{dim}\left(W_{N_{g}}^{1 \mathrm{D}}\right)=N_{g}\right)$, and $W_{N_{g}}^{3 \mathrm{D}}=W_{N_{g}}^{1 \mathrm{D}} \otimes W_{N_{g}}^{1 \mathrm{D}} \otimes W_{N_{g}}^{1 \mathrm{D}}$. Note that $W_{N_{g}}^{3 \mathrm{D}}$ is a subspace of $H_{\#}^{s}(\Gamma, \mathbb{C})$ of dimension $N_{g}^{3}$, for all $s \in \mathbb{R}$, and that if $N_{g}$ is odd,

$$
W_{N_{g}}^{3 \mathrm{D}}=\operatorname{Span}\left\{\left.e_{k}\left|k \in \mathcal{R}^{*}=\frac{2 \pi}{L} \mathbb{Z}^{3},\right| k\right|_{\infty} \leq \frac{2 \pi}{L}\left(\frac{N_{g}-1}{2}\right)\right\}
$$

It is then possible to define the interpolation projector $\mathcal{I}_{N_{g}}$ from $C_{\#}^{0}(\Gamma, \mathbb{C})$ onto $W_{N_{g}}^{3 \mathrm{D}}$ by $\left[\mathcal{I}_{N_{g}}(\phi)\right](x)=\phi(x)$ for all $x \in \mathcal{G}_{N_{g}}$. It holds

$$
\forall \phi \in C_{\#}^{0}(\Gamma, \mathbb{C}), \quad \int_{\Gamma} \mathcal{I}_{N_{g}}(\phi)=\sum_{x \in \mathcal{G}_{N_{g}} \cap \Gamma}\left(\frac{L}{N_{g}}\right)^{3} \phi(x) .
$$

The coefficients of the expansion of $\mathcal{I}_{N_{g}}(\phi)$ in the canonical basis of $W_{N_{g}}^{3 \mathrm{D}}$ is given by the discrete Fourier transform of $\phi$. In particular, when $N_{g}$ is odd, we have the simple relation

$$
\mathcal{I}_{N_{g}}(\phi)=|\Gamma|^{1 / 2} \sum_{\left.k \in \mathcal{R}^{*}|| k\right|_{\infty} \leq \frac{2 \pi}{L}\left(\frac{N_{g}-1}{2}\right)} \widehat{\phi}_{k}^{\mathrm{FFT}, N_{g}} e_{k} .
$$

It is easy to check that if $\phi$ is real-valued, then so is $\mathcal{I}_{N_{g}}(\phi)$.

We will assume in the sequel that $N_{g} \geq 4 N_{c}+1$. Using the properties of Gauss integration, we then have for all $v_{4 N_{c}} \in V_{4 N_{c}}$,

$$
\int_{\Gamma} v_{4 N_{c}}=\sum_{x \in \mathcal{G}_{N_{g}} \cap \Gamma}\left(\frac{L}{N_{g}}\right)^{3} v_{4 N_{c}}(x)=\int_{\Gamma} \mathcal{I}_{N_{g}}\left(v_{4 N_{c}}\right) .
$$


The following lemma collects some technical results which will be useful for the numerical analysis of the planewave discretization of orbital-free and Kohn-Sham models.

Lemma 2.1. Let $N_{c} \in \mathbb{N}^{*}$ and $N_{g} \in \mathbb{N}^{*}$ such that $N_{g} \geq 4 N_{c}+1$.

(1) Let $V$ be a function of $C_{\#}^{0}(\Gamma, \mathbb{C})$ and $v_{N_{c}}$ and $w_{N_{c}}$ be two functions of $V_{N_{c}}$. Then

$$
\begin{aligned}
& \int_{\Gamma} \mathcal{I}_{N_{g}}\left(V v_{N_{c}} w_{N_{c}}\right)=\int_{\Gamma} \mathcal{I}_{N_{g}}(V) v_{N_{c}} w_{N_{c}}, \\
& \left|\int_{\Gamma} \mathcal{I}_{N_{g}}\left(V\left|v_{N_{c}}\right|^{2}\right)\right| \leq\|V\|_{L^{\infty}}\left\|v_{N_{c}}\right\|_{L_{\#}^{2}}^{2} .
\end{aligned}
$$

(2) Let $s>3 / 2,0 \leq r \leq s$, and $V$ a function of $H_{\#}^{s}(\Gamma)$. Then,

$$
\begin{aligned}
&\left\|\left(1-\mathcal{I}_{N_{g}}\right)(V)\right\|_{H_{\#}^{r}} \leq C_{r, s} N_{g}^{-(s-r)}\|V\|_{H_{\#}^{s}}, \\
&\left\|\Pi_{2 N_{c}}\left(\mathcal{I}_{N_{g}}(V)\right)\right\|_{L_{\#}^{2}} \leq\left(\int_{\Gamma} \mathcal{I}_{N_{g}}\left(|V|^{2}\right)\right)^{1 / 2}, \\
&\left\|\Pi_{2 N_{c}}\left(\mathcal{I}_{N_{g}}(V)\right)\right\|_{H_{\#}^{s}} \leq\left(1+C_{s, s}\right)\|V\|_{H_{\#}^{s}},
\end{aligned}
$$

for constants $C_{r, s}$ independent of $V$. Besides if there exists $m>3$ and $C \in \mathbb{R}_{+}$such that $\left|\widehat{V}_{k}\right| \leq C|k|^{-m}$ for all $k \in \mathcal{R}^{*}$, then there exists a constant $C_{V}$ independent of $N_{c}$ and $N_{g}$ such that

$$
\left\|\Pi_{2 N_{c}}\left(1-\mathcal{I}_{N_{g}}\right)(V)\right\|_{H_{\#}^{r}} \leq C_{V} N_{c}^{r+3 / 2} N_{g}^{-m}
$$

(3) Let $\phi$ be a Borel function from $\mathbb{R}_{+}$to $\mathbb{R}$ such that there exists $C_{\phi} \in \mathbb{R}_{+}$for which $|\phi(t)| \leq C_{\phi}\left(1+t^{2}\right)$ for all $t \in \mathbb{R}_{+}$. Then, for all $v_{N_{c}} \in V_{N_{c}}$,

$$
\left|\int_{\Gamma} \mathcal{I}_{N_{g}}\left(\phi\left(\left|v_{N_{c}}\right|^{2}\right)\right)\right| \leq C_{\phi}\left(|\Gamma|+\left\|v_{N_{c}}\right\|_{L_{\#}^{4}}^{4}\right)
$$

Proof. For $z_{2 N_{c}} \in V_{2 N_{c}}$, it holds $\mathcal{I}_{N_{g}}(V) z_{2 N_{c}} \in W_{2 N_{g_{-1}}}$. It therefore follows from the properties of Gauss integration that

$$
\begin{aligned}
\int_{\Gamma} \mathcal{I}_{N_{g}}\left(V z_{2 N_{c}}\right) & =\sum_{x \in \mathcal{G}_{N_{g}} \cap \Gamma}\left(\frac{L}{N_{g}}\right)^{3} V(x) z_{2 N_{c}}(x) \\
& =\sum_{x \in \mathcal{G}_{N_{g}} \cap \Gamma}\left(\frac{L}{N_{g}}\right)^{3}\left(\mathcal{I}_{N_{g}}(V)\right)(x) z_{2 N_{c}}(x) \\
& =\int_{\Gamma} \mathcal{I}_{N_{g}}(V) z_{2 N_{c}} .
\end{aligned}
$$


The function $v_{N_{c}} w_{N_{c}}$ being in $V_{2 N_{c}},(2.5)$ is proved. Moreover, as $\left|v_{N_{c}}\right|^{2} \in V_{4 N_{c}}$, it holds

$$
\begin{aligned}
\left|\int_{\Gamma} \mathcal{I}_{N_{g}}\left(V\left|v_{N_{c}}\right|^{2}\right)\right| & =\left.\left|\sum_{x \in \mathcal{G}_{N_{g}} \cap \Gamma}\left(\frac{L}{N_{g}}\right)^{3} V(x)\right| v_{N_{c}}(x)\right|^{2} \mid \\
& \leq\left.\|V\|_{L^{\infty}}\left|\sum_{x \in \mathcal{G}_{N_{g}} \cap \Gamma}\left(\frac{L}{N_{g}}\right)^{3}\right| v_{N_{c}}(x)\right|^{2} \mid \\
& =\|V\|_{L^{\infty}} \int_{\Gamma}\left|v_{N_{c}}\right|^{2} .
\end{aligned}
$$

Hence (2.6). The estimate (2.7) is proved in [11], p. 272. To prove (2.8), we notice that

$$
\begin{aligned}
\left\|\Pi_{2 N_{c}}\left(\mathcal{I}_{N_{g}}(V)\right)\right\|_{L_{\#}^{2}}^{2} & \leq\left\|\mathcal{I}_{N_{g}}(V)\right\|_{L_{\#}^{2}}^{2} \\
& =\int_{\Gamma}\left(\mathcal{I}_{N_{g}}(V)\right)^{*}\left(\mathcal{I}_{N_{g}}(V)\right) \\
& =\sum_{x \in \mathcal{G}_{N_{g}} \cap \Gamma}\left(\frac{L}{N_{g}}\right)^{3}\left(\mathcal{I}_{N_{g}}(V)\right)(x)^{*}\left(\mathcal{I}_{N_{g}}(V)\right)(x) \\
& =\sum_{x \in \mathcal{G}_{N_{g}} \cap \Gamma}\left(\frac{L}{N_{g}}\right)^{3}|V(x)|^{2} \\
& =\int_{\Gamma} \mathcal{I}_{N_{g}}\left(|V|^{2}\right) .
\end{aligned}
$$

The bound (2.9) is a straightforward consequence of (2.7):

$$
\left\|\Pi_{2 N_{c}}\left(\mathcal{I}_{N_{g}}(V)\right)\right\|_{H_{\#}^{s}} \leq\left\|\mathcal{I}_{N_{g}}(V)\right\|_{H_{\#}^{s}} \leq\|V\|_{H_{\#}^{s}}+\left\|\left(1-\mathcal{I}_{N_{g}}\right)(V)\right\|_{H_{\#}^{s}} \leq\left(1+C_{s, s}\right)\|V\|_{H_{\#}^{s}} .
$$

Now, we notice that

$$
\begin{aligned}
\Pi_{2 N_{c}}\left(\mathcal{I}_{N_{g}}(V)\right) & =|\Gamma|^{1 / 2} \sum_{k \in \mathcal{R}^{*}|| k \mid \leq \frac{4 \pi}{L} N_{c}} \widehat{V}_{k}^{\mathrm{FFT}, N_{g}} e_{k} \\
& =\sum_{k \in \mathcal{R}^{*}|| k \mid \leq \frac{4 \pi}{L} N_{c}}\left(\sum_{K \in \mathcal{R}^{*}} \widehat{V}_{k+N_{g} K}\right) e_{k} .
\end{aligned}
$$

From (2.13), we obtain

$$
\begin{aligned}
\left\|\Pi_{2 N_{c}}\left(1-\mathcal{I}_{N_{g}}\right)(V)\right\|_{H_{\#}^{s}}^{2} & =\sum_{k \in \mathcal{R}^{*}|| k \mid \leq \frac{4 \pi}{L} N_{c}}\left(1+|k|^{2}\right)^{s}\left|\sum_{K \in \mathcal{R}^{*} \backslash\{0\}} \widehat{V}_{k+N_{g} K}\right|^{2} \\
& \leq\left(\sum_{k \in \mathcal{R}^{*}|| k \mid \leq \frac{4 \pi}{L} N_{c}}\left(1+|k|^{2}\right)^{s}\right) \max _{k \in \mathcal{R}^{*}|| k \mid \leq \frac{4 \pi}{L} N_{c}}\left|\sum_{K \in \mathcal{R}^{*} \backslash\{0\}} \widehat{V}_{k+N_{g} K}\right|^{2} .
\end{aligned}
$$

On the one hand,

$$
\sum_{k \in \mathcal{R}^{*}|| k \mid \leq \frac{4 \pi}{L} N_{c}}\left(1+|k|^{2}\right)^{s} \underset{N_{c} \rightarrow \infty}{\sim} \frac{32 \pi}{2 s+3}\left(\frac{4 \pi}{L}\right)^{2 s} N_{c}^{2 s+3},
$$


and on the other hand, we have for each $k \in \mathcal{R}^{*}$ such that $|k| \leq \frac{4 \pi}{L} N_{c}$,

$$
\begin{aligned}
\left|\sum_{K \in \mathcal{R}^{*} \backslash\{0\}} \widehat{V}_{k+N_{g} K}\right| & \leq C \sum_{K \in \mathcal{R}^{*} \backslash\{0\}} \frac{1}{\left|k+N_{g} K\right|^{m}} \\
& \leq C C_{0}\left(\frac{L}{2 \pi}\right)^{m} N_{g}^{-m}
\end{aligned}
$$

where

$$
C_{0}=\max _{y \in \mathbb{R}^{3}|| y \mid \leq 1 / 2} \sum_{K \in \mathbb{Z}^{3} \backslash\{0\}} \frac{1}{|y-K|^{m}} .
$$

The estimate (2.10) then easily follows. Let us finally prove (2.11). Using (2.3) and (2.4), we have

$$
\begin{aligned}
\left|\int_{\Gamma} \mathcal{I}_{N_{g}}\left(\phi\left(\left|v_{N_{c}}\right|^{2}\right)\right)\right| & =\left|\sum_{x \in \mathcal{G}_{N_{g}} \cap \Gamma}\left(\frac{L}{N_{g}}\right)^{3} \phi\left(\left|v_{N_{c}}(x)\right|^{2}\right)\right| \\
& \leq C_{\phi}\left|\sum_{x \in \mathcal{G}_{N_{g}} \cap \Gamma}\left(\frac{L}{N_{g}}\right)^{3}\left(1+\left|v_{N_{c}}(x)\right|^{4}\right)\right| \\
& =C_{\phi} \int_{\Gamma}\left(1+\left|v_{N_{c}}\right|^{4}\right)=C_{\phi}\left(|\Gamma|+\left\|v_{N_{c}}\right\|_{L_{\#}^{4}}^{4}\right) .
\end{aligned}
$$

This completes the proof of Lemma 2.1.

\section{Planewave approximation of the Periodic TFW model}

The periodic TFW problem reads as follows:

$$
I^{\mathrm{TFW}}=\inf \left\{\mathcal{E}^{\mathrm{TFW}}(\rho), \rho \in \mathfrak{R}_{\mathcal{N}}\right\},
$$

where

$$
\mathfrak{R}_{\mathcal{N}}=\left\{\rho \geq 0 \mid \sqrt{\rho} \in H_{\#}^{1}(\Gamma), \int_{\Gamma} \rho=\mathcal{N}\right\}
$$

is the set of admissible periodic densities, and where

$$
\mathcal{E}^{\mathrm{TFW}}(\rho)=\frac{C_{\mathrm{W}}}{2} \int_{\Gamma}|\nabla \sqrt{\rho}|^{2}+C_{\mathrm{TF}} \int_{\Gamma} \rho^{5 / 3}+\int_{\Gamma} \rho V^{\text {ion }}+\frac{1}{2} D_{\Gamma}(\rho, \rho) .
$$

The last term of the TFW energy models the periodic Coulomb energy: for $\rho$ and $\rho^{\prime}$ in $H_{\#}^{-1}(\Gamma)$,

$$
D_{\Gamma}\left(\rho, \rho^{\prime}\right):=4 \pi \sum_{k \in \mathcal{R}^{*} \backslash\{0\}}|k|^{-2} \widehat{\rho}_{k}^{*} \widehat{\rho}_{k}^{\prime} .
$$

We make the assumption that $V^{\text {ion }}$ is a $\mathcal{R}$-periodic potential such that

$$
\exists m>3, C \geq 0 \text { s.t. } \forall k \in \mathcal{R}^{*},\left|\widehat{V}_{k}^{\text {ion }}\right| \leq C|k|^{-m} .
$$


Note that this implies that $V^{\text {ion }}$ is in $H^{m-3 / 2-\epsilon}(\Gamma)$ for all $\epsilon>0$, hence in $C_{\#}^{0}(\Gamma)$ since $m-3 / 2-\epsilon>3 / 2$ for $\epsilon$ small enough. It is convenient to reformulate the TFW model in terms of $v=\sqrt{\rho}$. It can be easily seen that

$$
I^{\mathrm{TFW}}=\inf \left\{E^{\mathrm{TFW}}(v), v \in H_{\#}^{1}(\Gamma), \int_{\Gamma}|v|^{2}=\mathcal{N}\right\},
$$

where

$$
E^{\mathrm{TFW}}(v)=\frac{C_{\mathrm{W}}}{2} \int_{\Gamma}|\nabla v|^{2}+C_{\mathrm{TF}} \int_{\Gamma}|v|^{10 / 3}+\int_{\Gamma} V^{\mathrm{ion}}|v|^{2}+\frac{1}{2} D_{\Gamma}\left(|v|^{2},|v|^{2}\right) .
$$

Let $F(t)=C_{\mathrm{TF}} t^{5 / 3}$ and $f(t)=F^{\prime}(t)=\frac{5}{3} C_{\mathrm{TF}} t^{2 / 3}$. The function $F$ is in $C^{1}([0,+\infty)) \cap C^{\infty}((0,+\infty))$, is strictly convex on $[0,+\infty)$, and for all $\left(t_{1}, t_{2}\right) \in \mathbb{R}_{+} \times \mathbb{R}_{+}$,

$$
\left|f\left(t_{2}^{2}\right) t_{2}-f\left(t_{1}^{2}\right) t_{2}-2 f^{\prime}\left(t_{1}^{2}\right) t_{1}^{2}\left(t_{2}-t_{1}\right)\right| \leq \frac{70}{27} C_{\mathrm{TF}} \max \left(t_{1}^{1 / 3}, t_{2}^{1 / 3}\right)\left|t_{2}-t_{1}\right|^{2} .
$$

The first and second derivatives of $E^{\mathrm{TFW}}$ are respectively given by

$$
\begin{aligned}
& \left\langle E^{\mathrm{TFW}^{\prime}}(v), w\right\rangle_{H_{\#}^{-1}, H_{\#}^{1}}=2\left\langle\mathcal{H}_{|v|^{2}}^{\mathrm{TFW}} v, w\right\rangle, \\
& \left\langle E^{\mathrm{TFW}^{\prime \prime}}(v) w_{1}, w_{2}\right\rangle_{H_{\#}^{-1}, H_{\#}^{1}}=2\left\langle\mathcal{H}_{|v|^{2}}^{\mathrm{TFW}} w_{1}, w_{2}\right\rangle+4 D_{\Gamma}\left(v w_{1}, v w_{2}\right)+4 \int_{\Gamma} f^{\prime}\left(|v|^{2}\right)|v|^{2} w_{1} w_{2},
\end{aligned}
$$

where we have denoted by $\mathcal{H}_{\rho}^{\mathrm{TFW}}$ the TFW Hamiltonian associated with the density $\rho$

$$
\mathcal{H}_{\rho}^{\mathrm{TFW}}=-\frac{C_{\mathrm{W}}}{2} \Delta+f(\rho)+V^{\text {ion }}+V_{\rho}^{\text {Coulomb }}
$$

where

$$
V_{\rho}^{\text {Coulomb }}(x):=4 \pi \sum_{k \in \mathcal{R}^{*} \backslash\{0\}}|k|^{-2} \widehat{\rho}_{k} e_{k}(x)
$$

is the $\mathcal{R}$-periodic Coulomb potential generated by the $\mathcal{R}$-periodic charge distribution $\rho$. Recall that $V_{\rho}^{\text {Coulomb }}$ can also be defined as the unique solution in $H_{\#}^{1}(\Gamma)$ to

$$
\left\{\begin{array}{l}
-\Delta V_{\rho}^{\text {Coulomb }}=4 \pi\left(\rho-|\Gamma|^{-1} \int_{\Gamma} \rho\right) \\
\int_{\Gamma} V_{\rho}^{\text {Coulomb }}=0 .
\end{array}\right.
$$

Let us recall (see [27] and the proof of Lemma 2 in [6]) that

- (3.1) has a unique minimizer $\rho^{0}$, and that the minimizers of (3.3) are $u$ and $-u$, where $u=\sqrt{\rho^{0}}$;

- $u$ is in $H_{\#}^{m+1 / 2-\epsilon}(\Gamma)$ for each $\epsilon>0$ (hence in $C_{\#}^{2}(\Gamma)$ since $m+1 / 2-\epsilon>7 / 2$ for $\epsilon$ small enough);

- $u>0$ on $\mathbb{R}^{3}$;

- $u$ satisfies the Euler equation

$$
\mathcal{H}_{|u|^{2}}^{\mathrm{TFW}}(u)=-\frac{C_{\mathrm{W}}}{2} \Delta u+\left(\frac{5}{3} C_{\mathrm{TF}} u^{4 / 3}+V^{\text {ion }}+V_{u^{2}}^{\text {Coulomb }}\right) u=\lambda u
$$

for some $\lambda \in \mathbb{R}$, (the ground state eigenvalue of $\mathcal{H}_{\rho^{0}}^{\mathrm{TFW}}$, that is non-degenerate).

The planewave discretization of the TFW model is obtained by choosing:

(1) an energy cut-off $E_{\mathrm{c}}>0$ or, equivalently, a finite dimensional Fourier space $V_{N_{c}}$, the integer $N_{c}$ being related to $E_{\mathrm{c}}$ through the relation $N_{c}:=\left[\sqrt{2 E_{\mathrm{c}}} L / 2 \pi\right]$, 
(2) a cartesian grid $\mathcal{G}_{N_{g}}$ with step size $L / N_{g}$ where $N_{g} \in \mathbb{N}^{*}$ is such that $N_{g} \geq 4 N_{c}+1$, and by considering the finite dimensional minimization problem

$$
I_{N_{c}, N_{g}}^{\mathrm{TFW}}=\inf \left\{E_{N_{g}}^{\mathrm{TFW}}\left(v_{N_{c}}\right), v_{N_{c}} \in V_{N_{c}}, \int_{\Gamma}\left|v_{N_{c}}\right|^{2}=\mathcal{N}\right\}
$$

where

$$
\begin{aligned}
E_{N_{g}}^{\mathrm{TFW}}\left(v_{N_{c}}\right)= & \frac{C_{\mathrm{W}}}{2} \int_{\Gamma}\left|\nabla v_{N_{c}}\right|^{2}+C_{\mathrm{TF}} \int_{\Gamma} \mathcal{I}_{N_{g}}\left(\left|v_{N_{c}}\right|^{10 / 3}\right)+\int_{\Gamma} \mathcal{I}_{N_{g}}\left(V^{\mathrm{ion}}\right)\left|v_{N_{c}}\right|^{2} \\
& +\frac{1}{2} D_{\Gamma}\left(\left|v_{N_{c}}\right|^{2},\left|v_{N_{c}}\right|^{2}\right),
\end{aligned}
$$

$\mathcal{I}_{N_{g}}$ denoting the interpolation operator introduced in the previous section. The Euler equation associated with (3.6) can be written as a nonlinear eigenvalue problem

$$
\forall v_{N_{c}} \in V_{N_{c}}, \quad\left\langle\left(\widetilde{\mathcal{H}}_{\left|u_{N_{c}, N_{g}}\right|^{2}}^{\mathrm{TFW}, N_{g}}-\lambda_{N_{c}, N_{g}}\right) u_{N_{c}, N_{g}}, v_{N_{c}}\right\rangle_{H_{\#}^{-1}, H_{\#}^{1}}=0,
$$

where we have denoted by

$$
\widetilde{\mathcal{H}}_{\rho}^{\mathrm{TFW}, N_{g}}=-\frac{C_{\mathrm{W}}}{2} \Delta+\mathcal{I}_{N_{g}}\left(\frac{5}{3} C_{\mathrm{TF}} \rho^{2 / 3}+V^{\text {ion }}\right)+V_{\rho}^{\text {Coulomb }}
$$

the pseudospectral TFW Hamiltonian associated with the density $\rho$, and by $\lambda_{N_{c}, N_{g}}$ the Lagrange multiplier of the constraint $\int_{\Gamma}\left|v_{N_{c}}\right|^{2}=\mathcal{N}$. We therefore have

$$
-\frac{C_{\mathrm{W}}}{2} \Delta u_{N_{c}, N_{g}}+\Pi_{N_{c}}\left[\left(\mathcal{I}_{N_{g}}\left(\frac{5}{3} C_{\mathrm{TF}}\left|u_{N_{c}, N_{g}}\right|^{4 / 3}+V^{\text {ion }}\right)+V_{\left|u_{N_{c}, N_{g}}\right|^{2}}^{\text {Coulomb }}\right) u_{N_{c}, N_{g}}\right]=\lambda_{N_{c}, N_{g}} u_{N_{c}, N_{g}} .
$$

Under the condition that $N_{g} \geq 4 N_{c}+1$, we have for all $\phi \in C_{\#}^{0}(\Gamma)$,

$$
\forall(k, l) \in \mathcal{R}^{*} \times \mathcal{R}^{*} \text { s.t. }|k|,|l| \leq \frac{2 \pi}{L} N_{c}, \quad \int_{\Gamma} \mathcal{I}_{N_{g}}(\phi) e_{k}^{*} e_{l}=\widehat{\phi}_{k-l}^{\mathrm{FFT}},
$$

so that, $\widetilde{\mathcal{H}}_{u_{N_{c}, N_{g}}}^{\mathrm{TFW}}$ is defined on $V_{N_{c}}$ by the Fourier matrix

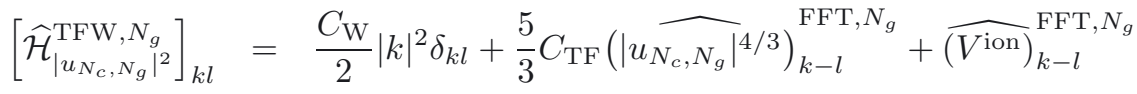

$$
\begin{aligned}
& +4 \pi \frac{\left(\mid{\widehat{u_{N_{c}, N_{g}}}}^{2}\right)_{k-l}^{\mathrm{FFT}, N_{g}}}{|k-l|^{2}}\left(1-\delta_{k l}\right),
\end{aligned}
$$

where, by convention, the last term of the right hand side is equal to zero for $k=l$.

We also introduce the variational approximation of (3.3)

$$
I_{N_{c}}^{\mathrm{TFW}}=\inf \left\{E^{\mathrm{TFW}}\left(v_{N_{c}}\right), v_{N_{c}} \in V_{N_{c}}, \int_{\Gamma}\left|v_{N_{c}}\right|^{2}=\mathcal{N}\right\} .
$$

Any minimizer $u_{N_{c}}$ to (3.7) satisfies the elliptic equation

$$
-\frac{C_{\mathrm{W}}}{2} \Delta u_{N_{c}}+\Pi_{N_{c}}\left[\frac{5}{3} C_{\mathrm{TF}}\left|u_{N_{c}}\right|^{4 / 3} u_{N_{c}}+V^{\mathrm{ion}} u_{N_{c}}+V_{\left|u_{N_{c}}\right|^{2}}^{\text {Coulomb }} u_{N_{c}}\right]=\lambda_{N_{c}} u_{N_{c}},
$$

for some $\lambda_{N_{c}} \in \mathbb{R}$. 
The following theorem provides optimal a priori error bounds for the planewave discretization of the periodic TFW model. Note that convergence results (without error estimates) were previously derived by Zhou [35].

Theorem 3.1. For each $N_{c} \in \mathbb{N}$, we denote by $u_{N_{c}}$ a minimizer to (3.7) such that $\left(u_{N_{c}}, u\right)_{L_{\#}^{2}} \geq 0$ and, for each $N_{c} \in \mathbb{N}$ and $N_{g} \geq 4 N_{c}+1$, we denote by $u_{N_{c}, N_{g}}$ a minimizer to (3.6) such that $\left(u_{N_{c}, N_{g}}, u\right)_{L_{\#}^{2}} \geq 0$. Then for $N_{c}$ large enough, $u_{N_{c}}$ and $u_{N_{c}, N_{g}}$ are unique, and the following estimates hold true

$$
\begin{aligned}
\left\|u_{N_{c}}-u\right\|_{H_{\#}^{s}} & \leq C_{s, \epsilon} N_{c}^{-(m-s+1 / 2-\epsilon)}, \\
\left|\lambda_{N_{c}}-\lambda\right| & \leq C_{\epsilon} N_{c}^{-(2 m-1-\epsilon)}, \\
\gamma\left\|u_{N_{c}}-u\right\|_{H_{\#}^{1}}^{2} \leq I_{N_{c}}^{\mathrm{TFW}}-I^{\mathrm{TFW}} & \leq C\left\|u_{N_{c}}-u\right\|_{H_{\#}^{1}}^{2}, \\
\left\|u_{N_{c}, N_{g}}-u_{N_{c}}\right\|_{H_{\#}^{s}} & \leq C_{s} N_{c}^{3 / 2+(s-1)_{+}} N_{g}^{-m}, \\
\left|\lambda_{N_{c}, N_{g}}-\lambda_{N_{c}}\right| & \leq C N_{c}^{3 / 2} N_{g}^{-m}, \\
\left|I_{N_{c}, N_{g}}^{\mathrm{TFW}}-I_{N_{c}}^{\mathrm{TFW}}\right| & \leq C N_{c}^{3 / 2} N_{g}^{-m},
\end{aligned}
$$

for all $-m+3 / 2<s<m+1 / 2$ and $\epsilon>0$, and for some constants $\gamma>0, C_{s, \epsilon} \geq 0, C_{\epsilon} \geq 0, C \geq 0$ and $C_{s} \geq 0$ independent of $N_{c}$ and $N_{g}$.

Remark 3.2. More complex orbital-free models have been proposed in the recent years [34], which are used to perform multimillion atom DFT calculations. Some of these models however are not well posed (the energy functional is not bounded from below [3]), and the others are not well understood from a mathematical point of view. For these reasons, we will not deal with those models in this article.

\subsection{A priori estimates for the variational approximation}

In this section, we prove the first part of Theorem 3.1, related to the variational approximation (3.7). The estimates (3.9), (3.10) and (3.11) originate from arguments already introduced in [6]. For brevity, we only recall the main steps of the proof and leave the details to the reader.

The difference between (3.3) and the problem dealt with in [6] is the presence of the Coulomb term $D_{\Gamma}\left(|v|^{2},|v|^{2}\right)$, for which the following estimates are available:

$$
\begin{aligned}
& 0 \leq D_{\Gamma}(\rho, \rho) \leq C\|\rho\|_{L_{\#}^{2}}^{2}, \quad \text { for all } \rho \in L_{\#}^{2}(\Gamma), \\
&\left|D_{\Gamma}(u v, u w)\right| \leq C\|v\|_{L_{\#}^{2}}\|w\|_{L_{\#}^{2}}, \quad \text { for all }(v, w) \in\left(L_{\#}^{2}(\Gamma)\right)^{2}, \\
&\left|D_{\Gamma}(\rho, v w)\right| \leq C\|\rho\|_{L_{\#}^{2}}\|v\|_{L_{\#}^{2}}^{2}\|w\|_{L_{\#}^{2}}, \quad \text { for all }(\rho, v, w) \in\left(L_{\#}^{2}(\Gamma)\right)^{3}, \\
&\left\|V_{\rho}^{\text {Coulomb }}\right\|_{L^{\infty}} \leq C\|\rho\|_{L_{\#}^{2}}, \quad \text { for all } \rho \in L_{\#}^{2}(\Gamma), \\
&\left\|V_{\rho}^{\text {Coulomb }}\right\|_{H_{\#}^{s+2}} \leq C\|\rho\|_{H_{\#}^{s}}, \quad \text { for all } \rho \in H_{\#}^{s}(\Gamma) .
\end{aligned}
$$

Note that (3.19) is valid for all $s \in \mathbb{R}$. Here and in the sequel, $C$ denotes a non-negative constant which may depend on $\Gamma, V^{\text {ion }}, \mathcal{N}$ and $s$, but not on the discretization parameters.

Using (3.15), (3.16) and the fact that $f^{\prime}>0$ on $(0,+\infty)$, we can then show (see the proof of Lemma 1 in [6]) that there exist $\beta>0, \gamma>0$ and $M \geq 0$ such that for all $v \in H_{\#}^{1}(\Gamma)$,

$$
\begin{aligned}
& 0 \leq\left\langle\left(\mathcal{H}_{\rho^{0}}^{\mathrm{TFW}}-\lambda\right) v, v\right\rangle_{H_{\#}^{-1}, H_{\#}^{1}} \leq M\|v\|_{H_{\#}^{1}}^{2}, \\
& \beta\|v\|_{H_{\#}^{1}}^{2} \leq\left\langle\left(E^{\mathrm{TFW}}(u)-2 \lambda\right) v, v\right\rangle_{H_{\#}^{-1}, H_{\#}^{1}} \leq M\|v\|_{H_{\#}^{1}}^{2},
\end{aligned}
$$


and for all $v \in H_{\#}^{1}(\Gamma)$ such that $\|v\|_{L_{\#}^{2}}=\mathcal{N}^{1 / 2}$ and $(v, u)_{L_{\#}^{2}} \geq 0$

$$
\gamma\|v-u\|_{H_{\#}^{1}}^{2} \leq\left\langle\left(\mathcal{H}_{\rho^{0}}^{\mathrm{TFW}}-\lambda\right)(v-u),(v-u)\right\rangle_{H_{\#}^{-1}, H_{\#}^{1}}
$$

Remarking that

$$
\begin{aligned}
E^{\mathrm{TFW}}\left(u_{N_{c}}\right)-E^{\mathrm{TFW}}(u)= & \left\langle\left(\mathcal{H}_{\rho^{0}}^{\mathrm{TFW}}-\lambda\right)\left(u_{N_{c}}-u\right),\left(u_{N_{c}}-u\right)\right\rangle_{H_{\#}^{-1}, H_{\#}^{1}}+\frac{1}{2} D_{\Gamma}\left(\left|u_{N_{c}}\right|^{2}-|u|^{2},\left|u_{N_{c}}\right|^{2}-|u|^{2}\right) \\
& +\int_{\Gamma} F\left(\left|u_{N_{c}}\right|^{2}\right)-F\left(|u|^{2}\right)-f\left(|u|^{2}\right)\left(\left|u_{N_{c}}\right|^{2}-|u|^{2}\right)
\end{aligned}
$$

and using (3.22), the positivity of the bilinear form $D_{\Gamma}$, and the convexity of the function $F$, we obtain that

$$
I_{N_{c}}^{\mathrm{TFW}}-I^{\mathrm{TFW}}=E^{\mathrm{TFW}}\left(u_{N_{c}}\right)-E^{\mathrm{TFW}}(u) \geq \gamma\left\|u_{N_{c}}-u\right\|_{H_{\#}^{1}}^{2}
$$

For each $N_{c} \in \mathbb{N}, \widetilde{u}_{N_{c}}=\mathcal{N}^{1 / 2} \Pi_{N_{c}} u /\left\|\Pi_{N_{c}} u\right\|_{L_{\#}^{2}}$ satisfies $\left(\widetilde{u}_{N_{c}}, u\right)_{L_{\#}^{2}} \geq 0$ and $\left\|\widetilde{u}_{N_{c}}\right\|_{L_{\#}^{2}}=\mathcal{N}^{1 / 2}$, and the sequence $\left(\widetilde{u}_{N_{c}}\right)_{N_{c} \in \mathbb{N}}$ converges to $u$ in $H_{\#}^{m+1 / 2-\epsilon}(\Gamma)$ for each $\epsilon>0$. As the functional $E^{\mathrm{TFW}}$ is continuous on $H_{\#}^{1}(\Gamma)$, we have

$$
\left\|u_{N_{c}}-u\right\|_{H_{\#}^{1}}^{2} \leq \gamma^{-1}\left(I_{N_{c}}^{\mathrm{TFW}}-I^{\mathrm{TFW}}\right) \leq \gamma^{-1}\left(E^{\mathrm{TFW}}\left(\widetilde{u}_{N_{c}}\right)-E^{\mathrm{TFW}}(u)\right) \underset{N_{c} \rightarrow \infty}{\longrightarrow} 0 .
$$

Hence, $\left(u_{N_{c}}\right)_{N_{c} \in \mathbb{N}}$ converges to $u$ in $H_{\#}^{1}(\Gamma)$, and we also have

$$
\begin{aligned}
\lambda_{N_{c}} & =\mathcal{N}^{-1}\left[\frac{C_{\mathrm{W}}}{2} \int_{\Gamma}\left|\nabla u_{N_{c}}\right|^{2}+\int_{\Gamma} f\left(\left|u_{N_{c}}\right|^{2}\right)\left|u_{N_{c}}\right|^{2}+\int_{\Gamma} V^{\mathrm{ion}}\left|u_{N_{c}}\right|^{2}+D_{\Gamma}\left(\left|u_{N_{c}}\right|^{2},\left|u_{N_{c}}\right|^{2}\right)\right] \\
\underset{N_{c}}{\longrightarrow} & \mathcal{N}^{-1}\left[\frac{C_{\mathrm{W}}}{2} \int_{\Gamma}|\nabla u|^{2}+\int_{\Gamma} f\left(|u|^{2}\right)|u|^{2}+\int_{\Gamma} V^{\mathrm{ion}}|u|^{2}+D_{\Gamma}\left(|u|^{2},|u|^{2}\right)\right] \\
& =\lambda .
\end{aligned}
$$

As $f\left(\left|u_{N_{c}}\right|^{2}\right) u_{N_{c}}+V^{\text {ion }} u_{N_{c}}+V_{\left|u_{N_{c}}\right|^{2}}^{\text {Coulomb }} u_{N_{c}}$ is bounded in $L_{\#}^{2}(\Gamma)$, uniformly in $N_{c}$, we deduce from (3.8) that the sequence $\left(u_{N_{c}}\right)_{N_{c} \in \mathbb{N}}$ is bounded in $H_{\#}^{2}(\Gamma)$, hence in $L^{\infty}(\Gamma)$. Now

$$
\begin{aligned}
& \Delta\left(u_{N_{c}}-u\right)=2 C_{\mathrm{W}}^{-1}\left[\Pi_{N_{c}}\left(f\left(\left|u_{N_{c}}\right|^{2}\right) u_{N_{c}}-f\left(|u|^{2}\right) u+V^{\text {ion }}\left(u_{N_{c}}-u\right)+V_{\left|u_{N_{c}}\right|^{2}}^{\text {Coulomb }} u_{N_{c}}-V_{|u|^{2}}^{\text {Coulomb }} u\right)\right. \\
& \left.-\left(1-\Pi_{N_{c}}\right)\left(f\left(|u|^{2}\right) u+V^{\text {ion }} u+V_{|u|^{2}}^{\text {Coulomb }} u\right)-\lambda_{N_{c}}\left(u_{N_{c}}-u\right)-\left(\lambda_{N_{c}}-\lambda\right) u\right] .
\end{aligned}
$$

Observing that the right-hand side goes to zero in $L_{\#}^{2}(\Gamma)$ when $N_{c}$ goes to infinity, we obtain that $\left(u_{N_{c}}\right)_{N_{c} \in \mathbb{N}}$ converges to $u$ in $H_{\#}^{2}(\Gamma)$, and therefore in $C_{\#}^{0,1 / 2}(\Gamma)$. In addition, we know from Harnack inequality [18] that $u>0$ in $\mathbb{R}^{3}$. Consequently, for $N_{c}$ large enough, the function $u_{N_{c}}$ (which is continuous and $\mathcal{R}$-periodic) is bounded away from 0 , uniformly in $N_{c}$. As $f \in C^{\infty}(0,+\infty)$, one can see by a simple bootstrap argument that the convergence of $\left(u_{N_{c}}\right)_{N_{c} \in \mathbb{N}}$ to $u$ also holds in $H_{\#}^{m+1 / 2-\epsilon}(\Gamma)$ for each $\epsilon>0$. The upper bound in (3.11) is obtained from (3.23), remarking that

$$
\begin{aligned}
0 & \leq \int_{\Gamma} F\left(\left|u_{N_{c}}\right|^{2}\right)-F\left(|u|^{2}\right)-f\left(|u|^{2}\right)\left(\left|u_{N_{c}}\right|^{2}-|u|^{2}\right) \\
& \leq \frac{35}{9} C_{\mathrm{TF}} \int_{\Gamma} \max \left(\left|u_{N_{c}}\right|^{4 / 3},|u|^{4 / 3}\right)\left|u_{N_{c}}-u\right|^{2} \\
& \leq \frac{35}{9} C_{\mathrm{TF}}\left(\max _{N_{c} \in \mathbb{N}}\left\|u_{N_{c}}\right\|_{L^{\infty}}\right)^{4 / 3}\left\|u_{N_{c}}-u\right\|_{L_{\#}^{2}}^{2},
\end{aligned}
$$


and that

$$
\begin{aligned}
0 & \leq D_{\Gamma}\left(\left|u_{N_{c}}\right|^{2}-|u|^{2},\left|u_{N_{c}}\right|^{2}-|u|^{2}\right) \leq C\left\|\left|u_{N_{c}}\right|^{2}-|u|^{2}\right\|_{L_{\#}^{2}}^{2} \\
& \leq 4 C\left(\max _{N_{c} \in \mathbb{N}}\left\|u_{N_{c}}\right\|_{L^{\infty}}\right)^{2}\left\|u_{N_{c}}-u\right\|_{L_{\#}^{2}}^{2} .
\end{aligned}
$$

The uniqueness of $u_{N_{c}}$ for $N_{c}$ large enough can then be checked as follows. First, $\left(u_{N_{c}}, \lambda_{N_{c}}\right)$ satisfies the variational equation

$$
\forall v_{N_{c}} \in V_{N_{c}}, \quad\left\langle\left(\mathcal{H}_{\left|u_{N_{c}}\right|^{2}}^{\mathrm{TFW}}-\lambda_{N_{c}}\right) u_{N_{c}}, v_{N_{c}}\right\rangle_{H_{\#}^{-1}, H_{\#}^{1}}=0 .
$$

Therefore $\lambda_{N_{c}}$ is the variational approximation in $V_{N_{c}}$ of some eigenvalue of $\mathcal{H}_{\left|u_{N_{c}}\right|^{2}}^{\mathrm{TFW}}$. As $\left(u_{N_{c}}\right)_{N_{c} \in \mathbb{N}}$ converges to $u$ in $L^{\infty}(\Gamma), \mathcal{H}_{\left|u_{N_{c}}\right|^{2}}^{\mathrm{TFW}}-\mathcal{H}_{\rho^{0}}^{\mathrm{TFW}}$ converges to 0 in operator norm. Consequently, the $n$th eigenvalue of $\mathcal{H}_{\left|u_{N_{c}}\right|^{2}}^{\mathrm{TFW}}$ converges to the $n$th eigenvalue of $\mathcal{H}_{\rho^{0}}^{\mathrm{TFW}}$ when $N_{c}$ goes to infinity, the convergence being uniform in $n$. Together with the fact that the sequence $\left(\lambda_{N_{c}}\right)_{N_{c} \in \mathbb{N}}$ converges to $\lambda$, the non-degenerate ground state eigenvalue of $\mathcal{H}_{\rho^{0}}^{\mathrm{TFW}}$, this implies that for $N_{c}$ large enough, $\lambda_{N_{c}}$ is the ground state eigenvalue of $\mathcal{H}_{\left|u_{N_{c}}\right|^{2}}^{\mathrm{TFW}}$ in $V_{N_{c}}$ and for all $v_{N_{c}} \in V_{N_{c}}$ such that $\left\|v_{N_{c}}\right\|_{L_{\#}^{2}}=\mathcal{N}^{1 / 2}$ and $\left(v_{N_{c}}, u_{N_{c}}\right)_{L_{\#}^{2}} \geq 0$,

$$
\begin{aligned}
E^{\mathrm{TFW}}\left(v_{N_{c}}\right)-E^{\mathrm{TFW}}\left(u_{N_{c}}\right)= & \left\langle\left(\mathcal{H}_{\left|u_{N_{c}}\right|^{2}}^{\mathrm{TFW}}-\lambda_{N_{c}}\right)\left(v_{N_{c}}-u_{N_{c}}\right),\left(v_{N_{c}}-u_{N_{c}}\right)\right\rangle_{H_{\#}^{-1}, H_{\#}^{1}} \\
& +\frac{1}{2} D_{\Gamma}\left(\left|v_{N_{c}}\right|^{2}-\left|u_{N_{c}}\right|^{2},\left|v_{N_{c}}\right|^{2}-\left|u_{N_{c}}\right|^{2}\right) \\
& +\int_{\Gamma} F\left(\left|v_{N_{c}}\right|^{2}\right)-F\left(\left|u_{N_{c}}\right|^{2}\right)-f\left(\left|u_{N_{c}}\right|^{2}\right)\left(\left|v_{N_{c}}\right|^{2}-\left|u_{N_{c}}\right|^{2}\right) \\
\geq & \left\langle\left(\mathcal{H}_{\left|u_{N_{c}}\right|^{2}}^{\mathrm{TFW}}-\lambda_{N_{c}}\right)\left(v_{N_{c}}-u_{N_{c}}\right),\left(v_{N_{c}}-u_{N_{c}}\right)\right\rangle_{H_{\#}^{-1}, H_{\#}^{1}} \\
\geq & \frac{\gamma}{2}\left\|v_{N_{c}}-u_{N_{c}}\right\|_{H_{\#}^{1}}^{2} .
\end{aligned}
$$

It easily follows that for $N_{c}$ large enough, (3.7) has a unique minimizer $u_{N_{c}}$ such that $\left(u_{N_{c}}, u\right)_{L_{\#}^{2}} \geq 0$.

Let us now establish the rates of convergence of $\left|\lambda_{N_{c}}-\lambda\right|$ and $\left\|u_{N_{c}}-u\right\|_{H_{\#}^{s}}$. First,

$$
\lambda_{N_{c}}-\lambda=\mathcal{N}^{-1}\left[\left\langle\left(\mathcal{H}_{|u|^{2}}^{\mathrm{TFW}}-\lambda\right)\left(u_{N_{c}}-u\right),\left(u_{N_{c}}-u\right)\right\rangle_{H_{\#}^{-1}, H_{\#}^{1}}+\int_{\Gamma} w_{N_{c}}\left(u_{N_{c}}-u\right)\right]
$$

with

$$
w_{N_{c}}=\frac{f\left(\left|u_{N_{c}}\right|^{2}\right)-f\left(|u|^{2}\right)}{u_{N_{c}}-u}\left|u_{N_{c}}\right|^{2}+V_{\left|u_{N_{c}}\right|^{2}}^{\text {Coulomb }}\left(u_{N_{c}}+u\right) .
$$

As $u_{N_{c}}$ is bounded away from 0 and $f \in C^{\infty}((0,+\infty))$, the function $w_{N_{c}}$ is uniformly bounded in $H_{\#}^{m-3 / 2-\epsilon}(\Gamma)$ (at least for $N_{c}$ large enough). We therefore obtain that for all $0 \leq r<m-3 / 2$, there exists a constant $C_{r} \in \mathbb{R}_{+}$ such that for all $N_{c}$ large enough,

$$
\left|\lambda_{N_{c}}-\lambda\right| \leq C_{r}\left(\left\|u_{N_{c}}-u\right\|_{H_{\#}^{1}}^{2}+\left\|u_{N_{c}}-u\right\|_{H_{\#}^{-r}}\right) .
$$

In order to evaluate the $H_{\#}^{1}$-norm of the error $\left(u_{N_{c}}-u\right)$, we first notice that

$$
\forall v_{N_{c}} \in V_{N_{c}}, \quad\left\|u_{N_{c}}-u\right\|_{H_{\#}^{1}} \leq\left\|u_{N_{c}}-v_{N_{c}}\right\|_{H_{\#}^{1}}+\left\|v_{N_{c}}-u\right\|_{H_{\#}^{1}},
$$


and that

$$
\begin{aligned}
\left\|u_{N_{c}}-v_{N_{c}}\right\|_{H_{\#}^{1}}^{2} \leq & \beta^{-1}\left\langle\left(E^{\mathrm{TFW}^{\prime \prime}}(u)-2 \lambda\right)\left(u_{N_{c}}-v_{N_{c}}\right),\left(u_{N_{c}}-v_{N_{c}}\right)\right\rangle_{H_{\#}^{-1}, H_{\#}^{1}} \\
= & \beta^{-1}\left(\left\langle\left(E^{\mathrm{TFW}^{\prime \prime}}(u)-2 \lambda\right)\left(u_{N_{c}}-u\right),\left(u_{N_{c}}-v_{N_{c}}\right)\right\rangle_{H_{\#}^{-1}, H_{\#}^{1}}\right. \\
& \left.+\left\langle\left(E^{\mathrm{TFW}^{\prime \prime}}(u)-2 \lambda\right)\left(u-v_{N_{c}}\right),\left(u_{N_{c}}-v_{N_{c}}\right)\right\rangle_{H_{\#}^{-1}, H_{\#}^{1}}\right) .
\end{aligned}
$$

For all $z_{N_{c}} \in V_{N_{c}}$,

$$
\begin{aligned}
\left\langle\left(E^{\mathrm{TFW}}{ }^{\prime \prime}(u)-2 \lambda\right)\left(u_{N_{c}}-u\right), z_{N_{c}}\right\rangle_{H_{\#}^{-1}, H_{\#}^{1}}= & -2 \int_{\Gamma}\left[f\left(\left|u_{N_{c}}\right|^{2}\right) u_{N_{c}}-f\left(|u|^{2}\right) u_{N_{c}}-2 f^{\prime}\left(|u|^{2}\right)|u|^{2}\left(u_{N_{c}}-u\right)\right] z_{N_{c}} \\
& -2 D_{\Gamma}\left(\left(u_{N_{c}}-u\right)\left(u_{N_{c}}+u\right),\left(u_{N_{c}}-u\right) z_{N_{c}}\right) \\
& -2 D_{\Gamma}\left(\left(u_{N_{c}}-u\right)^{2}, u z_{N_{c}}\right)+2\left(\lambda_{N_{c}}-\lambda\right) \int_{\Gamma} u_{N_{c}} z_{N_{c}} .
\end{aligned}
$$

On the other hand, we have for all $v_{N_{c}} \in V_{N_{c}}$ such that $\left\|v_{N_{c}}\right\|_{L_{\#}^{2}}=\mathcal{N}^{1 / 2}$,

$$
\int_{\Gamma} u_{N_{c}}\left(u_{N_{c}}-v_{N_{c}}\right)=\mathcal{N}-\int_{\Gamma} u_{N_{c}} v_{N_{c}}=\frac{1}{2}\left\|u_{N_{c}}-v_{N_{c}}\right\|_{L_{\#}^{2}}^{2} .
$$

Using (3.4), (3.17), (3.26) with $r=0$ and the above equality, we therefore obtain for all $v_{N_{c}} \in V_{N_{c}}$ such that $\left\|v_{N_{c}}\right\|_{L_{\#}^{2}}=\mathcal{N}^{1 / 2}$,

$$
\begin{aligned}
\left|\left\langle\left(E^{\mathrm{TFW}^{\prime \prime}}(u)-2 \lambda\right)\left(u_{N_{c}}-u\right),\left(u_{N_{c}}-v_{N_{c}}\right)\right\rangle_{H_{\#}^{-1}, H_{\#}^{1}}\right| \leq & C\left(\left\|u_{N_{c}}-u\right\|_{H_{\#}^{1}}^{2}\left\|u_{N_{c}}-v_{N_{c}}\right\|_{H_{\#}^{1}}\right. \\
& \left.+\left(\left\|u_{N_{c}}-u\right\|_{H_{\#}^{1}}^{2}+\left\|u_{N_{c}}-u\right\|_{L_{\#}^{2}}\right)\left\|u_{N_{c}}-v_{N_{c}}\right\|_{L_{\#}^{2}}^{2}\right) .
\end{aligned}
$$

Therefore, for $N_{c}$ large enough, we have for all $v_{N_{c}} \in V_{N_{c}}$ such that $\left\|v_{N_{c}}\right\|_{L_{\#}^{2}}=\mathcal{N}^{1 / 2}$,

$$
\left\|u_{N_{c}}-v_{N_{c}}\right\|_{H_{\#}^{1}} \leq C\left(\left\|u_{N_{c}}-u\right\|_{H_{\#}^{1}}^{2}+\left\|v_{N_{c}}-u\right\|_{H_{\#}^{1}}\right) .
$$

Together with (3.27), this shows that there exists $N \in \mathbb{N}$ and $C \in \mathbb{R}_{+}$such that for all $N_{c} \geq N$,

$$
\forall v_{N_{c}} \in V_{N_{c}} \text { s.t. }\left\|v_{N_{c}}\right\|_{L_{\#}^{2}}=\mathcal{N}^{1 / 2}, \quad\left\|u_{N_{c}}-u\right\|_{H_{\#}^{1}} \leq C\left\|v_{N_{c}}-u\right\|_{H_{\#}^{1}} .
$$

By a classical argument (see e.g. the proof of Theorem 1 in [6]), we deduce from (2.2) and the above inequality that

$$
\left\|u_{N_{c}}-u\right\|_{H_{\#}^{1}} \leq C \min _{v_{N_{c}} \in V_{N_{c}}}\left\|v_{N_{c}}-u\right\|_{H_{\#}^{1}} \leq C_{1, \epsilon} N_{c}^{-(m-1 / 2-\epsilon)},
$$

for some constant $C_{1, \epsilon}$ independent of $N_{c}$. This completes the proof of the estimate in the $H_{\#}^{1}$-norm. We proceed with the analysis of the $L_{\#}^{2}$-norm.

For $w \in L_{\#}^{2}(\Gamma)$, we denote by $\psi_{w}$ the unique solution to the adjoint problem

$$
\left\{\begin{array}{l}
\text { find } \psi_{w} \in u^{\perp} \text { such that } \\
\forall v \in u^{\perp}, \quad\left\langle\left(E^{\mathrm{TFW}}{ }^{\prime \prime}(u)-2 \lambda\right) \psi_{w}, v\right\rangle_{H_{\#}^{-1}, H_{\#}^{1}}=\langle w, v\rangle_{H_{\#}^{-1}, H_{\#}^{1}}
\end{array}\right.
$$


where

$$
u^{\perp}=\left\{v \in H_{\#}^{1}(\Gamma) \mid \int_{\Gamma} u v=0\right\}
$$

The function $\psi_{w}$ is solution to the elliptic equation

$$
\begin{gathered}
-\frac{C_{\mathrm{W}}}{2} \Delta \psi_{w}+\left(V^{\text {ion }}+V_{u^{2}}^{\text {Coulomb }}+f\left(u^{2}\right)+2 f^{\prime}\left(u^{2}\right) u^{2}-\lambda\right) \psi_{w}+2 V_{u \psi_{w}}^{\text {Coulomb }} u \\
=2\left(\int_{\Gamma} f^{\prime}\left(u^{2}\right) u^{3} \psi_{w}+D_{\Gamma}\left(u^{2}, u \psi_{w}\right)\right) u+\frac{1}{2}\left(w-(w, u)_{L_{\#}^{2}} u\right),
\end{gathered}
$$

from which we deduce that if $w \in H_{\#}^{r}(\Gamma)$ for some $0 \leq r<m-3 / 2$, then $\psi_{w} \in H_{\#}^{r+2}(\Gamma)$ and

$$
\left\|\psi_{w}\right\|_{H_{\#}^{r+2}} \leq C_{r}\|w\|_{H_{\#}^{r}}
$$

for some constant $C_{r}$ independent of $w$. Let $u_{N_{c}}^{*}$ be the orthogonal projection, for the $L_{\#}^{2}$ inner product, of $u_{N_{c}}$ on the affine space $\left\{v \in L_{\#}^{2}(\Gamma) \mid \int_{\Gamma} u v=\mathcal{N}\right\}$. One has

$$
u_{N_{c}}^{*} \in H_{\#}^{1}(\Gamma), \quad u_{N_{c}}^{*}-u \in u^{\perp}, \quad u_{N_{c}}^{*}-u_{N_{c}}=\frac{1}{2 \mathcal{N}}\left\|u_{N_{c}}-u\right\|_{L_{\#}^{2}}^{2} u
$$

from which we infer that

$$
\begin{aligned}
& \left\|u_{N_{c}}-u\right\|_{L_{\#}^{2}}^{2}=\int_{\Gamma}\left(u_{N_{c}}-u\right)\left(u_{N_{c}}^{*}-u\right)+\int_{\Gamma}\left(u_{N_{c}}-u\right)\left(u_{N_{c}}-u_{N_{c}}^{*}\right) \\
& =\int_{\Gamma}\left(u_{N_{c}}-u\right)\left(u_{N_{c}}^{*}-u\right)-\frac{1}{2 \mathcal{N}}\left\|u_{N_{c}}-u\right\|_{L_{\#}^{2}}^{2} \int_{\Gamma}\left(u_{N_{c}}-u\right) u \\
& =\int_{\Gamma}\left(u_{N_{c}}-u\right)\left(u_{N_{c}}^{*}-u\right)+\frac{1}{2 \mathcal{N}}\left\|u_{N_{c}}-u\right\|_{L_{\#}^{2}}^{2}\left(\mathcal{N}-\int_{\Gamma} u_{N_{c}} u\right) \\
& =\int_{\Gamma}\left(u_{N_{c}}-u\right)\left(u_{N_{c}}^{*}-u\right)+\frac{1}{4 \mathcal{N}}\left\|u_{N_{c}}-u\right\|_{L_{\#}^{2}}^{4} \\
& =\left\langle u_{N_{c}}-u, u_{N_{c}}^{*}-u\right\rangle_{H_{\#}^{-1}, H_{\#}^{1}}+\frac{1}{4 \mathcal{N}^{\prime}}\left\|u_{N_{c}}-u\right\|_{L_{\#}^{2}}^{4} \\
& =\left\langle\left(E^{\mathrm{TFW}^{\prime \prime}}(u)-2 \lambda\right) \psi_{u_{N_{c}}-u}, u_{N_{c}}^{*}-u\right\rangle_{H_{\#}^{-1}, H_{\#}^{1}}+\frac{1}{4 \mathcal{N}}\left\|u_{N_{c}}-u\right\|_{L_{\#}^{2}}^{4} \\
& =\left\langle\left(E^{\mathrm{TFW}}(u)-2 \lambda\right)\left(u_{N_{c}}-u\right), \psi_{u_{N_{c}}-u}\right\rangle_{H_{\#}^{-1}, H_{\#}^{1}}+\frac{1}{4 \mathcal{N}^{\prime}}\left\|u_{N_{c}}-u\right\|_{L_{\#}^{2}}^{4} \\
& +\frac{1}{2 \mathcal{N}}\left\|u_{N_{c}}-u\right\|_{L_{\#}^{2}}^{2}\left\langle\left(E^{\mathrm{TFW}}{ }^{\prime \prime}(u)-2 \lambda\right) u, \psi_{u_{N_{c}}-u}\right\rangle_{H_{\#}^{-1}, H_{\#}^{1}} \\
& =\left\langle\left(E^{\mathrm{TFW}}{ }^{\prime \prime}(u)-2 \lambda\right)\left(u_{N_{c}}-u\right), \psi_{u_{N_{c}}-u}\right\rangle_{H_{\#}^{-1}, H_{\#}^{1}}+\frac{1}{4 \mathcal{N}^{\prime}}\left\|u_{N_{c}}-u\right\|_{L_{\#}^{2}}^{4} \\
& +\frac{2}{\mathcal{N}}\left\|u_{N_{c}}-u\right\|_{L_{\#}^{2}}^{2}\left[\int_{\Gamma} f^{\prime}\left(u^{2}\right) u^{3} \psi_{u_{N_{c}}-u}+D_{\Gamma}\left(u^{2}, u \psi_{u_{N_{c}}-u}\right)\right] .
\end{aligned}
$$


For all $\psi_{N_{c}} \in V_{N_{c}}$, it therefore holds

$$
\begin{aligned}
\left\|u_{N_{c}}-u\right\|_{L_{\#}^{2}}^{2}= & \left\langle\left(E^{\mathrm{TFW}^{\prime \prime}}(u)-2 \lambda\right)\left(u_{N_{c}}-u\right), \psi_{u_{N_{c}}-u}-\psi_{N_{c}}\right\rangle_{H_{\#}^{-1}, H_{\#}^{1}} \\
& +\left\langle\left(E^{\mathrm{TFW}^{\prime \prime}}(u)-2 \lambda\right)\left(u_{N_{c}}-u\right), \psi_{N_{c}}\right\rangle_{H_{\#}^{-1}, H_{\#}^{1}}+\frac{1}{4 \mathcal{N}}\left\|u_{N_{c}}-u\right\|_{L_{\#}^{2}}^{4} \\
& +\frac{2}{\mathcal{N}}\left\|u_{N_{c}}-u\right\|_{L_{\#}^{2}}^{2}\left[\int_{\Gamma} f^{\prime}\left(u^{2}\right) u^{3} \psi_{u_{N_{c}}-u}+D_{\Gamma}\left(u^{2}, u \psi_{u_{N_{c}}-u}\right)\right] .
\end{aligned}
$$

Using (3.4), (3.17), (3.26) with $r=0$ and (3.30), we obtain that for all $\psi_{N_{c}} \in V_{N_{c}} \cap u^{\perp}$,

$$
\begin{aligned}
\left|\left\langle\left(E^{\mathrm{TFW}}(u)-2 \lambda\right)\left(u_{N_{c}}-u\right), \psi_{N_{c}}\right\rangle_{H_{\#}^{-1}, H_{\#}^{1}}\right| \leq & C\left(\left\|u_{N_{c}}-u\right\|_{H_{\#}^{1}}^{2}\right. \\
& \left.+\left\|u_{N_{c}}-u\right\|_{L_{\#}^{2}}\left(\left\|u_{N_{c}}-u\right\|_{H_{\#}^{1}}^{2}+\left\|u_{N_{c}}-u\right\|_{L_{\#}^{2}}\right)\right)\left\|\psi_{N_{c}}\right\|_{H_{\#}^{1}} .
\end{aligned}
$$

Let us denote by $\Pi_{V_{N_{c}} \cap u^{\perp}}^{1}$ the orthogonal projector on $V_{N_{c}} \cap u^{\perp}$ for the $H_{\#}^{1}$ inner product and by $\psi_{N_{c}}^{0}=$ $\Pi_{V_{N_{c}} \cap u^{\perp}}^{1} \psi_{u_{N_{c}}-u}$. Noticing that

$$
\left\|\psi_{N_{c}}^{0}\right\|_{H_{\#}^{1}} \leq\left\|\psi_{u_{N_{c}}-u}\right\|_{H_{\#}^{1}} \leq \beta^{-1} M\left\|u_{N_{c}}-u\right\|_{L_{\#}^{2}}
$$

we obtain from (3.21), (3.34) and (3.35) that there exists $N \in \mathbb{N}$ and $C \in \mathbb{R}_{+}$such that for all $N_{c} \geq N$,

$$
\left\|u_{N_{c}}-u\right\|_{L_{\#}^{2}}^{2} \leq C\left(\left\|u_{N_{c}}-u\right\|_{L_{\#}^{2}}\left\|u_{N_{c}}-u\right\|_{H_{\#}^{1}}^{2}+\left\|u_{N_{c}}-u\right\|_{H_{\#}^{1}}\left\|\psi_{u_{N_{c}}-u}-\psi_{N_{c}}^{0}\right\|_{H_{\#}^{1}}\right) .
$$

Lastly, for all $v \in u^{\perp}$ and all $N_{c} \in \mathbb{N}^{*}$

$$
\left\|v-\Pi_{V_{N_{c}} \cap u^{\perp}}^{1} v\right\|_{H_{\#}^{1}} \leq\left(1+\frac{\mathcal{N}^{1 / 2} L^{5 / 2}}{2 \pi N_{c} \int_{\Gamma} u}\right)\left\|v-\Pi_{N_{c}} v\right\|_{H_{\#}^{1}}
$$

so that, in view of $(2.2)$ and $(3.33)$

$$
\begin{aligned}
\left\|\psi_{u_{N_{c}}-u}-\psi_{N_{c}}^{0}\right\|_{H_{\#}^{1}} & \leq C\left\|\psi_{u_{N_{c}}-u}-\Pi_{N_{c}} \psi_{u_{N_{c}}-u}\right\|_{H_{\#}^{1}} \\
& \leq C N_{c}^{-1}\left\|\psi_{u_{N_{c}}-u}\right\|_{H_{\#}^{2}} \\
& \leq C N_{c}^{-1}\left\|u_{N_{c}}-u\right\|_{L_{\#}^{2}}^{2}
\end{aligned}
$$

Therefore,

$$
\begin{aligned}
\left\|u_{N_{c}}-u\right\|_{L_{\#}^{2}} & \leq C\left(\left\|u_{N_{c}}-u\right\|_{H_{\#}^{1}}^{2}+N_{c}^{-1}\left\|u_{N_{c}}-u\right\|_{H_{\#}^{1}}\right) \\
& \leq C_{0, \epsilon} N_{c}^{-(m+1 / 2-\epsilon)} .
\end{aligned}
$$

By means of the inverse inequality

$$
\forall v_{N_{c}} \in V_{N_{c}}, \quad\left\|v_{N_{c}}\right\|_{H_{\#}^{r}} \leq\left(\frac{2 \pi}{L}\right)^{(r-s)} N_{c}^{r-s}\left\|v_{N_{c}}\right\|_{H_{\#}^{s}},
$$


which holds true for all $s \leq r$ and all $N_{c} \geq 1$, we obtain that

$$
\left\|u_{N_{c}}-u\right\|_{H_{\#}^{s}} \leq C_{s, \epsilon} N_{c}^{-(m-s+1 / 2-\epsilon)} \quad \text { for all } 0 \leq s<m+1 / 2 .
$$

To complete the first part of the proof of Theorem 3.1, we still have to compute the $H_{\#}^{-r}$-norm of the error $\left(u_{N_{c}}-u\right)$ for $0<r<m-3 / 2$. Let $w \in H_{\#}^{r}(\Gamma)$. Proceeding as above we obtain

$$
\begin{aligned}
\int_{\Gamma} w\left(u_{N_{c}}-u\right)= & \left\langle\left(E^{\mathrm{TFW}}(u)-2 \lambda\right)\left(u_{N_{c}}-u\right), \Pi_{V_{N_{c}} \cap u^{\perp}}^{1} \psi_{w}\right\rangle_{H_{\#}^{-1}, H_{\#}^{1}} \\
& +\left\langle\left(E^{\mathrm{TFW}}(u)-2 \lambda\right)\left(u_{N_{c}}-u\right), \psi_{w}-\Pi_{V_{N_{c}} \cap u^{\perp}}^{1} \psi_{w}\right\rangle_{H_{\#}^{-1}, H_{\#}^{1}}+\frac{2}{\mathcal{N}}\left\|u_{N_{c}}-u\right\|_{L_{\#}^{2}}^{2} \\
& \times\left[\int_{\Gamma} f^{\prime}\left(u^{2}\right) u^{3} \psi_{w}+D_{\Gamma}\left(u^{2}, u \psi_{w}\right)\right]-\frac{1}{2 \mathcal{N}^{\prime}}\left\|u_{N_{c}}-u\right\|_{L_{\#}^{2}}^{2} \int_{\Gamma} u w .
\end{aligned}
$$

Combining (3.21), (3.33), (3.35), (3.36), (3.38) and (3.39), we obtain that there exists a constant $C \in \mathbb{R}_{+}$such that for all $N_{c}$ large enough and all $w \in H_{\#}^{r}(\Gamma)$,

$$
\begin{aligned}
\int_{\Gamma} w\left(u_{N_{c}}-u\right) & \leq C^{\prime}\left(\left\|u_{N_{c}}-u\right\|_{H_{\#}^{1}}^{2}+N_{c}^{-(r+1)}\left\|u_{N_{c}}-u\right\|_{H_{\#}^{1}}\right)\|w\|_{H_{\#}^{r}} \\
& \leq C_{-r, \epsilon} N_{c}^{-(m+r+1 / 2-\epsilon)}\|w\|_{H_{\#}^{r}} .
\end{aligned}
$$

Therefore

$$
\left\|u_{N_{c}}-u\right\|_{H_{\#}^{-r}}=\sup _{w \in H_{\#}^{r}(\Gamma) \backslash\{0\}} \frac{\int_{\Gamma} w\left(u_{N_{c}}-u\right)}{\|w\|_{H_{\#}^{r}}} \leq C_{-r, \epsilon} N_{c}^{-(m+r+1 / 2-\epsilon)},
$$

for some constant $C_{-r, \epsilon} \in \mathbb{R}_{+}$independent of $N_{c}$. Using (3.26), (3.31) and (3.40), we end up with

$$
\left|\lambda_{N_{c}}-\lambda\right| \leq C_{\epsilon} N_{c}^{-(2 m-1-\epsilon)} .
$$

\subsection{A priori estimates for the full discretization}

Let us now turn to the pseudospectral approximation (3.6) of (3.3). First, we notice that

$$
\frac{C_{\mathrm{W}}}{2}\left\|\nabla u_{N_{c}, N_{g}}\right\|_{L_{\#}^{2}}^{2}-\left\|V^{\mathrm{ion}}\right\|_{L^{\infty}} \mathcal{N} \leq E_{N_{g}}^{\mathrm{TFW}}\left(u_{N_{c}, N_{g}}\right) \leq E_{N_{g}}^{\mathrm{TFW}}\left(\mathcal{N}^{1 / 2}|\Gamma|^{-1 / 2}\right) \leq C_{\mathrm{TF}} \mathcal{N}^{5 / 3}|\Gamma|^{-2 / 3}+\left\|V^{\mathrm{ion}}\right\|_{L^{\infty}} \mathcal{N},
$$

from which we infer that $u_{N_{c}, N_{g}}$ is uniformly bounded in $H_{\#}^{1}(\Gamma)$. We then see that

$$
\lambda_{N_{c}, N_{g}}=\mathcal{N}^{-1}\left[\frac{C_{\mathrm{W}}}{2} \int_{\Gamma}\left|\nabla u_{N_{c}, N_{g}}\right|^{2}+\int_{\Gamma} \mathcal{I}_{N_{g}}\left(V^{\mathrm{ion}}\left|u_{N_{c}, N_{g}}\right|^{2}+f\left(\left|u_{N_{c}, N_{g}}\right|^{2}\right)\left|u_{N_{c}, N_{g}}\right|^{2}\right)+D_{\Gamma}\left(\left|u_{N_{c}, N_{g}}\right|^{2},\left|u_{N_{c}, N_{g}}\right|^{2}\right)\right] .
$$

Using (2.6), (2.11) and (3.15), we obtain that $\lambda_{N_{c}, N_{g}}$ also is uniformly bounded. Now,

$$
\begin{aligned}
\Delta u_{N_{c}, N_{g}}= & 2 C_{\mathrm{W}}^{-1} \Pi_{N_{c}}\left(\mathcal{I}_{N_{g}}\left(f\left(\left|u_{N_{c}, N_{g}}\right|^{2}\right) u_{N_{c}, N_{g}}\right)\right)+2 C_{\mathrm{W}}^{-1} \Pi_{N_{c}}\left(\mathcal{I}_{N_{g}}\left(V^{\text {ion }} u_{N_{c}, N_{g}}\right)\right) \\
& +2 C_{\mathrm{W}}^{-1} \Pi_{N_{c}}\left(V_{\left|u_{N_{c}, N_{g}}\right|^{2}}^{\text {Coulomb }} u_{N_{c}, N_{g}}\right)-2 C_{\mathrm{W}}^{-1} \lambda_{N_{c}, N_{g}} u_{N_{c}, N_{g}},
\end{aligned}
$$


and we deduce from (2.4), (2.6) and (2.8) that

$$
\begin{aligned}
\left\|\Pi_{N_{c}}\left(\mathcal{I}_{N_{g}}\left(f\left(\left|u_{N_{c}, N_{g}}\right|^{2}\right) u_{N_{c}, N_{g}}\right)\right)\right\|_{L_{\#}^{2}} & \leq\left(\int_{\Gamma}\left(\mathcal{I}_{N_{g}}\left(f\left(\left|u_{N_{c}, N_{g}}\right|^{2}\right)\right)\right)^{2}\left|u_{N_{c}, N_{g}}\right|^{2}\right)^{1 / 2} \\
& =\left(\sum_{x \in \mathcal{G}_{N_{g}} \cap \Gamma}\left(\frac{L}{N_{g}}\right)^{3} f\left(\left|u_{N_{c}, N_{g}}(x)\right|^{2}\right)^{2}\left|u_{N_{c}, N_{g}}(x)\right|^{2}\right)^{1 / 2} \\
& \leq \frac{5}{3} C_{\mathrm{TF}}\left\|u_{N_{c}, N_{g}}\right\|_{L^{\infty}}^{1 / 3}\left(\sum_{x \in \mathcal{G}_{N_{g}} \cap \Gamma}\left(\frac{L}{N_{g}}\right)^{3}\left|u_{N_{c}, N_{g}}(x)\right|^{4}\right)^{1 / 2} \\
& =\frac{5}{3} C_{\mathrm{TF}}\left\|u_{N_{c}, N_{g}}\right\|_{L^{\infty}}^{1 / 3}\left\|u_{N_{c}, N_{g}}\right\|_{L_{\#}^{4}}^{2},
\end{aligned}
$$

and that

$$
\begin{aligned}
\left\|\Pi_{N_{c}}\left(\mathcal{I}_{N_{g}}\left(V^{\mathrm{ion}} u_{N_{c}, N_{g}}\right)\right)\right\|_{L_{\#}^{2}} & \leq\left\|\Pi_{2 N_{c}}\left(\mathcal{I}_{N_{g}}\left(V^{\mathrm{ion}} u_{N_{c}, N_{g}}\right)\right)\right\|_{L_{\#}^{2}} \\
& \leq\left(\int_{\Gamma} \mathcal{I}_{N_{g}}\left(\left|V^{\mathrm{ion}}\right|^{2}\left|u_{N_{c}, N_{g}}\right|^{2}\right)\right)^{1 / 2} \\
& \leq\left\|V^{\mathrm{ion}}\right\|_{L^{\infty} \mathcal{N}^{1 / 2}}
\end{aligned}
$$

Besides, using (3.18),

$$
\left\|\Pi_{N_{c}}\left(V_{\left|u_{N_{c}, N_{g}}\right|^{2}}^{\text {Coulomb }} u_{N_{c}, N_{g}}\right)\right\|_{L_{\#}^{2}} \leq\left\|V_{\left|u_{N_{c}, N_{g}}\right|^{2}}^{\text {Coulomb }} u_{N_{c}, N_{g}}\right\|_{L_{\#}^{2}} \leq \mathcal{N}^{1 / 2}\left\|V_{\left|u_{N_{c}, N_{g}}\right|^{2}}^{\text {Coulomb }}\right\|_{L^{\infty}} \leq \mathcal{N}^{1 / 2}\left\|u_{N_{c}, N_{g}}\right\|_{L_{\#}^{4}}^{2} .
$$

As $u_{N_{c}, N_{g}}$ is uniformly bounded in $H_{\#}^{1}(\Gamma)$, and therefore in $L_{\#}^{4}(\Gamma)$, we get

$$
\left\|u_{N_{c}, N_{g}}\right\|_{H_{\#}^{2}}=\left(\left\|u_{N_{c}, N_{g}}\right\|_{L_{\#}^{2}}^{2}+\left\|\Delta u_{N_{c}, N_{g}}\right\|_{L_{\#}^{2}}^{2}\right)^{1 / 2} \leq C\left(1+\left\|u_{N_{c}, N_{g}}\right\|_{L^{\infty}}^{1 / 3}\right) \leq C\left(1+\left\|u_{N_{c}, N_{g}}\right\|_{H_{\#}^{2}}^{1 / 3}\right) .
$$

Therefore $u_{N_{c}, N_{g}}$ is uniformly bounded in $H_{\#}^{2}(\Gamma)$, hence in $L^{\infty}\left(\mathbb{R}^{3}\right)$.

Returning to (3.41) and using (2.9), (3.2), and a bootstrap argument, we conclude that $u_{N_{c}, N_{g}}$ is in fact uniformly bounded in $H_{\#}^{7 / 2+\epsilon}(\Gamma)$.

Next, using (3.24),

$$
\begin{aligned}
\frac{\gamma}{2}\left\|u_{N_{c}, N_{g}}-u_{N_{c}}\right\|_{H_{\#}^{1}}^{2} \leq & E^{\mathrm{TFW}}\left(u_{N_{c}, N_{g}}\right)-E^{\mathrm{TFW}}\left(u_{N_{c}}\right) \\
= & E_{N_{g}}^{\mathrm{TFW}}\left(u_{N_{c}, N_{g}}\right)-E_{N_{g}}^{\mathrm{TFW}}\left(u_{N_{c}}\right)+\int_{\Gamma}\left(\left(1-\mathcal{I}_{N_{g}}\right)(V)\right)\left(\left|u_{N_{c}, N_{g}}\right|^{2}-\left|u_{N_{c}}\right|^{2}\right) \\
& +\int_{\Gamma}\left(1-\mathcal{I}_{N_{g}}\right)\left(F\left(\left|u_{N_{c}, N_{g}}\right|^{2}\right)-F\left(\left|u_{N_{c}}\right|^{2}\right)\right) \\
\leq & \int_{\Gamma}\left(\left(1-\mathcal{I}_{N_{g}}\right)(V)\right)\left(\left|u_{N_{c}, N_{g}}\right|^{2}-\left|u_{N_{c}}\right|^{2}\right)+\int_{\Gamma}\left(1-\mathcal{I}_{N_{g}}\right)\left(F\left(\left|u_{N_{c}, N_{g}}\right|^{2}\right)-F\left(\left|u_{N_{c}}\right|^{2}\right)\right) .
\end{aligned}
$$

Let $g\left(t, t^{\prime}\right)=\frac{F\left(t^{\prime 2}\right)-F\left(t^{2}\right)}{t^{\prime}-t}$. For $N_{c}$ large enough, $u_{N_{c}}$ is uniformly bounded away from zero; besides, both $u_{N_{c}}$ and $u_{N_{c}, N_{g}}$ are uniformly bounded in $H_{\#}^{7 / 2+\epsilon}(\Gamma)$. Therefore, $g\left(u_{N_{c}}, u_{N_{c}, N_{g}}\right)$ is uniformly bounded in $H_{\#}^{7 / 2+\epsilon}(\Gamma)$. This implies that the Fourier coefficients of $g\left(u_{N_{c}}, u_{N_{c}, N_{g}}\right)$ go to zero faster that $|k|^{-7 / 2}$, which in turn implies, 
using (2.5) and (2.10), that

$$
\begin{aligned}
\left|\int_{\Gamma}\left(1-\mathcal{I}_{N_{g}}\right)\left(F\left(\left|u_{N_{c}, N_{g}}\right|^{2}\right)-F\left(\left|u_{N_{c}}\right|^{2}\right)\right)\right| & =\left|\int_{\Gamma}\left(1-\mathcal{I}_{N_{g}}\right)\left(g\left(u_{N_{c}}, u_{N_{c}, N_{g}}\right)\right)\left(u_{N_{c}, N_{g}}-u_{N_{c}}\right)\right| \\
& \leq\left\|\Pi_{N_{c}}\left(\left(1-\mathcal{I}_{N_{g}}\right)\left(g\left(u_{N_{c}}, u_{N_{c}, N_{g}}\right)\right)\right)\right\|_{L_{\#}^{2}}\left\|u_{N_{c}, N_{g}}-u_{N_{c}}\right\|_{L_{\#}^{2}} \\
& \leq C N_{c}^{3 / 2} N_{g}^{-7 / 2}\left\|u_{N_{c}, N_{g}}-u_{N_{c}}\right\|_{L_{\#}^{2}} .
\end{aligned}
$$

On the other hand,

$$
\begin{aligned}
\left|\int_{\Gamma}\left(\left(1-\mathcal{I}_{N_{g}}\right)(V)\right)\left(\left|u_{N_{c}, N_{g}}\right|^{2}-\left|u_{N_{c}}\right|^{2}\right)\right| & \leq\left\|\Pi_{2 N_{c}}\left(\left(1-\mathcal{I}_{N_{g}}\right)(V)\right)\right\|_{L_{\#}^{2}}\left\|u_{N_{c}, N_{g}}+u_{N_{c}}\right\|_{L^{\infty}}\left\|u_{N_{c}, N_{g}}-u_{N_{c}}\right\|_{L_{\#}^{2}} \\
& \leq C N_{c}^{3 / 2} N_{g}^{-m}\left\|u_{N_{c}, N_{g}}-u_{N_{c}}\right\|_{L_{\#}^{2} .}
\end{aligned}
$$

Therefore,

$$
\left\|u_{N_{c}, N_{g}}-u_{N_{c}}\right\|_{H_{\#}^{1}} \leq C N_{c}^{3 / 2} N_{g}^{-7 / 2} .
$$

We then deduce from (3.43) and the inverse inequality (3.37) that $\left(u_{N_{c}, N_{g}}\right)_{N_{c}, N_{g} \geq 4 N_{c}+1}$ converges to $u$ in $H_{\#}^{2}(\Gamma)$, and therefore in $L^{\infty}\left(\mathbb{R}^{3}\right)$. It follows that for $N_{c}$ large enough, $u_{N_{c}, N_{g}}$ is bounded away from zero, which, together with (3.41), implies that $\left(u_{N_{c}, N_{g}}\right)_{N_{c}, N_{g} \geq 4 N_{c}+1}$ is bounded in $H_{\#}^{m+1 / 2-\epsilon}(\Gamma)$. The estimates (3.42) and (3.43) can therefore be improved, yielding

$$
\left|\int_{\Gamma}\left(1-\mathcal{I}_{N_{g}}\right)\left(F\left(\left|u_{N_{c}, N_{g}}\right|^{2}\right)-F\left(\left|u_{N_{c}}\right|^{2}\right)\right)\right| \leq C N_{c}^{3 / 2} N_{g}^{-(m+1 / 2-\epsilon)}\left\|u_{N_{c}, N_{g}}-u_{N_{c}}\right\|_{L_{\#}^{2}},
$$

and

$$
\left\|u_{N_{c}, N_{g}}-u_{N_{c}}\right\|_{H_{\#}^{1}} \leq C N_{c}^{3 / 2} N_{g}^{-m}
$$

We deduce (3.12) from the inverse inequality (3.37). For $N_{c}$ large enough, $u_{N_{c}, N_{g}}$ is bounded away from zero, so that $f\left(\left|u_{N_{c}, N_{g}}\right|^{2}\right)$ is uniformly bounded in $H_{\#}^{m+1 / 2-\epsilon}(\Gamma)$. Therefore, the $k$ th Fourier coefficient of $\left(V^{\text {ion }}+f\left(\left|u_{N_{c}, N_{g}}\right|^{2}\right)\right)$ is bounded by $C|k|^{-m}$ where the constant $C$ does not depend on $N_{c}$ and $N_{g}$. Using the equality

$$
\begin{aligned}
\lambda_{N_{c}, N_{g}}-\lambda_{N_{c}}= & \mathcal{N}^{-1}\left[\left\langle\left(\mathcal{H}_{\left|u_{N_{c}}\right|^{2}}^{\mathrm{TFW}}-\lambda_{N_{c}}\right)\left(u_{N_{c}, N_{g}}-u_{N_{c}}\right),\left(u_{N_{c}, N_{g}}-u_{N_{c}}\right)\right\rangle_{H_{\#}^{-1}, H_{\#}^{1}}\right. \\
& -\int_{\Gamma}\left(1-\mathcal{I}_{N_{g}}\right)\left(V^{\mathrm{ion}}+f\left(\left|u_{N_{c}, N_{g}}\right|^{2}\right)\right)\left|u_{N_{c}, N_{g}}\right|^{2} \\
& \left.+D_{\Gamma}\left(\left|u_{N_{c}, N_{g}}\right|^{2},\left|u_{N_{c}, N_{g}}\right|^{2}-\left|u_{N_{c}}\right|^{2}\right)+\int_{\Gamma}\left(f\left(\left|u_{N_{c}, N_{g}}\right|^{2}\right)-f\left(\left|u_{N_{c}}\right|^{2}\right)\right)\left|u_{N_{c}, N_{g}}\right|^{2}\right],
\end{aligned}
$$

(3.12) and (3.17), we obtain (3.13). A similar calculation leads to (3.14). 
Lastly, we have for all $v_{N_{c}} \in V_{N_{c}}$,

$$
\begin{aligned}
E_{N_{g}}^{\mathrm{TFW}}\left(v_{N_{c}}\right)-E_{N_{g}}^{\mathrm{TFW}}\left(u_{N_{c}, N_{g}}\right)= & \left\langle\left(\widetilde{\mathcal{H}}_{u_{N_{c}, N_{g}}}^{\mathrm{TFW}}-\lambda_{N_{c}, N_{g}}\right)\left(v_{N_{c}}-u_{N_{c}, N_{g}}\right),\left(v_{N_{c}}-u_{N_{c}, N_{g}}\right)\right\rangle_{H_{\#}^{-1}, H_{\#}^{1}} \\
& +\frac{1}{2} D_{\Gamma}\left(\left|v_{N_{c}}\right|^{2}-\left|u_{N_{c}, N_{g}}\right|^{2},\left|v_{N_{c}}\right|^{2}-\left|u_{N_{c}, N_{g}}\right|^{2}\right) \\
& +\sum_{x \in \mathcal{G}_{N_{g}} \cap \Gamma}\left(\frac{L}{N_{g}}\right)^{3}\left(F\left(\left|v_{N_{c}}(x)\right|^{2}\right)-F\left(\left|u_{N_{c}}(x)\right|^{2}\right)\right. \\
& \left.-f\left(\left|u_{N_{c}}(x)\right|^{2}\right)\left(\left|v_{N_{c}}(x)\right|^{2}-\left|u_{N_{c}}(x)\right|^{2}\right)\right) \\
\geq & \left\langle\left(\widetilde{\mathcal{H}}_{u_{N_{c}, N_{g}}^{\mathrm{TFW}}}^{\mathrm{TW}}-\lambda_{N_{c}, N_{g}}\right)\left(v_{N_{c}}-u_{N_{c}, N_{g}}\right),\left(v_{N_{c}}-u_{N_{c}, N_{g}}\right)\right\rangle_{H_{\#}^{-1}, H_{\#}^{1}} .
\end{aligned}
$$

As $u_{N_{c}, N_{g}}$ converges to $u$ in $H_{\#}^{2}(\Gamma)$, the operator $\widetilde{\mathcal{H}}_{\mid u_{N_{c},\left.N_{g}\right|^{2}}}^{\mathrm{TFW}, N_{g}}-\mathcal{H}_{\rho^{0}}^{\mathrm{TFW}}$ converges to zero in operator norm. Reasoning as in the proof of the uniqueness of $u_{N_{c}}$, we obtain that for $N_{c}$ large enough and $N_{g} \geq 4 N_{c}+1$, we have for all $v_{N_{c}} \in V_{N_{c}}$ such that $\left\|v_{N_{c}}\right\|_{L_{\#}^{2}}=\mathcal{N}^{1 / 2}$ and $\left(v_{N_{c}}, u_{N_{c}}\right)_{L_{\#}^{2}} \geq 0$,

$$
\left\langle\left(\widetilde{\mathcal{H}}_{u_{N_{c}, N_{g}}}^{\mathrm{TFW}}-\lambda_{N_{c}, N_{g}}\right)\left(v_{N_{c}}-u_{N_{c}, N_{g}}\right),\left(v_{N_{c}}-u_{N_{c}, N_{g}}\right)\right\rangle_{H_{\#}^{-1}, H_{\#}^{1}} \geq \frac{\gamma}{2}\left\|v_{N_{c}}-u_{N_{c}, N_{g}}\right\|_{H_{\#}^{1}}^{2} .
$$

Thus the uniqueness of $u_{N_{c}, N_{g}}$ for $N_{c}$ large enough is proven.

\section{Planewave approximation of the Periodic Kohn-Sham LDA model}

The periodic Kohn-Sham LDA model with norm-conserving pseudopotentials [32] leads to the constrained optimization problem

$$
I^{\mathrm{KS}}=\inf \left\{E^{\mathrm{KS}}(\Phi), \Phi \in \mathcal{M}\right\},
$$

where

$$
\mathcal{M}=\left\{\Phi=\left(\phi_{1}, \ldots, \phi_{N}\right)^{T} \in\left(H_{\#}^{1}(\Gamma)\right)^{N} \mid \int_{\Gamma} \phi_{i} \phi_{j}=\delta_{i j}\right\}
$$

$N$ being the number of valence electron pairs in the simulation cell, and where

$$
E^{\mathrm{KS}}(\Phi)=\sum_{i=1}^{N} \int_{\Gamma}\left|\nabla \phi_{i}\right|^{2}+\int_{\Gamma} \rho_{\Phi} V_{\text {local }}+2 \sum_{i=1}^{N}\left\langle\phi_{i}\left|V_{\mathrm{nl}}\right| \phi_{i}\right\rangle+J\left(\rho_{\Phi}\right)+E_{\mathrm{xc}}^{\mathrm{LDA}}\left(\rho_{\Phi}\right) .
$$

The density $\rho_{\Phi}$ associated with $\Phi$, the Coulomb energy $J\left(\rho_{\Phi}\right)$ and the LDA exchange-correlation energy $E_{\mathrm{xc}}^{\mathrm{LDA}}\left(\rho_{\Phi}\right)$ are respectively defined as

$$
\begin{aligned}
\rho_{\Phi}(x) & =2 \sum_{i=1}^{N}\left|\phi_{i}(x)\right|^{2}, \\
J\left(\rho_{\Phi}\right) & =\frac{1}{2} D_{\Gamma}\left(\rho_{\Phi}, \rho_{\Phi}\right)=\left.2 \pi \sum_{k \in \mathcal{R}^{*} \backslash\{0\}}|k|^{-2}\left|\widehat{\left(\rho_{\Phi}\right)}\right|_{k}\right|^{2} \\
E_{\mathrm{xc}}^{\mathrm{LDA}}\left(\rho_{\Phi}\right) & =\int_{\Gamma} e_{\mathrm{xc}}^{\mathrm{LDA}}\left(\rho_{\mathrm{c}}(x)+\rho_{\Phi}(x)\right) \mathrm{d} x,
\end{aligned}
$$

where $\rho_{\mathrm{c}} \geq 0$ is the nonlinear core correction and where $e_{\mathrm{xc}}^{\mathrm{LDA}}(\rho)$ is an approximation of the exchange-correlation energy per unit volume in a uniform electron gas with charge density $\rho$ [15].

The local and nonlocal contributions to the pseudopotential model the interactions between valence electrons on the one hand, and nuclei and core electrons on the other hand. The local contribution is represented 
by a function $V_{\text {local }} \in C_{\#}^{0}(\Gamma)$ (and therefore defines a bounded self-adjoint operator on $L_{\#}^{2}(\Gamma)$ ); the nonlocal contribution is represented by the bounded self-adjoint operator $V_{\mathrm{nl}}$ defined on $L_{\#}^{2}(\Gamma)$ by

$$
V_{\mathrm{nl}} \phi=\sum_{j=1}^{M}\left(\xi_{j}, \phi\right)_{L_{\#}^{2}} \xi_{j},
$$

where the functions $\xi_{j}$ are regular enough functions of $L_{\#}^{2}(\Gamma)$. In all that follows, we will assume that

$$
\exists m>3, C \geq 0 \text { s.t. } \forall k \in \mathcal{R}^{*},\left|\widehat{\left(\widehat{V_{\text {local }}}\right)_{k}}\right| \leq C|k|^{-m}
$$

and that

$$
\forall 1 \leq j \leq M, \quad \forall \epsilon>0, \quad \xi_{j} \in H_{\#}^{m-3 / 2-\epsilon}(\Gamma) .
$$

Troullier-Martins pseudopotentials [32] constitute a popular class of pseudopotentials for which the Fourier coefficients $\left(\widehat{V_{\text {local }}}\right)_{k}$ decay as $|k|^{-m}$ with $m=5$.

The exact LDA exchange-correlation function (defined as the exchange-correlation energy per unit volume in a uniform electron gas) cannot be made explicit in terms of usual functions. Although this function is smooth $\left(C^{\infty}\right)$ on the open set $(0,+\infty)$, DFT simulation softwares make use of approximate functions which are $C^{\infty}$ on $\left(0, \rho_{*}\right) \cup\left(\rho_{*},+\infty\right)$ but only $C^{1,1}$ in the neighborhood of the density $\rho_{*}:=3 /(4 \pi)$ (atomic units) [15]. In order not to deteriorate the convergence rate of the pseudospectral approximation, it is better to resort to more regular approximations of the exact LDA exchange-correlation function (see [7]). We will assume here that

$$
\begin{aligned}
& \text { the function } \rho \mapsto e_{\mathrm{xc}}^{\mathrm{LDA}}(\rho) \text { is in } C^{1}([0,+\infty)) \cap C^{3}((0,+\infty)), \\
& e_{\mathrm{xc}}^{\mathrm{LDA}}(0)=0, \quad \frac{d e_{\mathrm{xc}}^{\mathrm{LDA}}}{d \rho}(0)=0,
\end{aligned}
$$

and that there exists $0<\alpha \leq 1$ and $C \in \mathbb{R}_{+}$such that

$$
\forall \rho \in \mathbb{R}_{+} \backslash\{0\}, \quad\left|\frac{d^{2} e_{\mathrm{xc}}^{\mathrm{LDA}}}{d \rho^{2}}(\rho)\right|+\left|\rho \frac{d^{3} e_{\mathrm{xc}}^{\mathrm{LDA}}}{d \rho^{3}}(\rho)\right| \leq C\left(1+\rho^{\alpha-1}\right) .
$$

Note that the $\mathrm{X} \alpha$ exchange-correlation functional $\left(e_{\mathrm{xc}}^{\mathrm{X} \alpha}(\rho)=-C_{\mathrm{X}} \rho^{4 / 3}\right.$, where $C_{\mathrm{X}}>0$ is a given constant) satisfies the assumptions (4.5)-(4.7) with $\alpha=1 / 3$. These assumptions are also satisfied by the exact exchangecorrelation functional [15]. Lastly, we assume that

$$
\rho_{\mathrm{c}} \in H_{\#}^{m-3 / 2-\epsilon}(\Gamma) .
$$

It is easy to prove that under assumptions $(4.3)-(4.8),(4.1)$ has a minimizer $\Phi^{0}=\left(\phi_{1}^{0}, \ldots, \phi_{N}^{0}\right)^{T}$ with density $\rho^{0}=\rho_{\Phi^{0}}$. Indeed, any minimizing sequence of (4.1) then is bounded in $H_{\#}^{1}(\Gamma)$ and therefore converges (up to extraction) weakly in $H_{\#}^{1}(\Gamma)$ and strongly in $L_{\#}^{1}(\Gamma) \cap L_{\#}^{6-\epsilon}(\Gamma)$. This allows one to pass to the limit in the orthonormality constraints and to the limit inferior in the energy, and conclude to the existence of a minimizer. We refer the reader to [9], Chapter 2, for a pedagogical introduction to these techniques. The situation is more difficult when the Kohn-Sham problem is set on the whole space $\mathbb{R}^{3}$. In this case, the Kohn-Sham problem does not always have a minimizer, for some charge can escape to infinity. Such a loss of compactness of the minimizing sequences is observed for very negative ions. On the other hand, it is known that for neutral or positively charged molecular systems, the Kohn-Sham LDA problem set on the whole space $\mathbb{R}^{3}$ does have a minimizer $[1,23]$. 
Let us introduce the Kohn-Sham Hamiltonian

$$
\mathcal{H}_{\rho^{0}}^{\mathrm{KS}}=-\frac{1}{2} \Delta+\left(V_{\text {local }}+V_{\rho^{0}}^{\text {Coulomb }}+\frac{d e_{\mathrm{xc}}^{\mathrm{LDA}}}{d \rho}\left(\rho_{\mathrm{c}}+\rho^{0}\right)\right)+V_{\mathrm{nl}}=h+\mathcal{V}_{\rho^{0}},
$$

where

$$
h=-\frac{1}{2} \Delta+V_{\text {local }}+V_{\mathrm{nl}}
$$

and

$$
\mathcal{V}_{\rho^{0}}=V_{\rho^{0}}^{\text {Coulomb }}+\frac{d e_{\mathrm{xc}}^{\mathrm{LDA}}}{d \rho}\left(\rho_{\mathrm{c}}+\rho^{0}\right)
$$

where $V$ Coulomb is defined by (3.5).

We notice that $E^{\mathrm{KS}^{\prime}}\left(\Phi^{0}\right)=4 \mathcal{H}_{\rho^{0}}^{\mathrm{KS}} \Phi^{0}$ in $\left(H_{\#}^{-1}(\Gamma)\right)^{N}$ and thus the Euler equations associated with the minimization problem (4.1) read

$$
\forall 1 \leq i \leq N, \quad \mathcal{H}_{\rho^{0}}^{\mathrm{KS}} \phi_{i}^{0}=\sum_{j=1}^{N} \lambda_{i j}^{0} \phi_{j}^{0},
$$

where the $N \times N$ matrix $\Lambda_{N}^{0}=\left(\lambda_{i j}^{0}\right)$, which is the Lagrange multiplier of the matrix constraint $\int_{\Gamma} \phi_{i} \phi_{j}=\delta_{i j}$, is symmetric. Equations (4.11) also read

$$
-\Delta \phi_{i}^{0}=f_{i}
$$

where

$$
f_{i}=2\left(\sum_{j=1}^{N} \lambda_{i j}^{0} \phi_{j}^{0}-\left(V_{\text {local }}+V_{\rho^{0}}^{\text {Coulomb }}+\frac{d e_{\mathrm{xc}}^{\mathrm{LDA}}}{d \rho}\left(\rho_{\mathrm{c}}+\rho^{0}\right)\right) \phi_{i}^{0}-V_{\mathrm{nl}} \phi_{i}^{0}\right) .
$$

The regularity assumptions on $V_{\text {local }}, e_{\mathrm{xc}}^{\mathrm{LDA}}, \rho_{\mathrm{c}}$ and on the functions $\xi_{j}$ imply that each $f_{i}$ is in $L_{\#}^{2}(\Gamma)$, which by elliptic regularity implies in turn that the minimizer $\Phi^{0}$ is in $\left(H_{\#}^{2}(\Gamma)\right)^{N}$.

In order to obtain more regularity on $\Phi^{0}$, and thereby convergence estimates of the planewave approximation in stronger Sobolev norms, we need to make additional assumptions on the function $e_{\mathrm{xc}}^{\mathrm{LDA}}$. Basically, we need to choose $e_{\mathrm{xc}}^{\mathrm{LDA}}$ regular enough for $\frac{d e_{\mathrm{xc}}^{\mathrm{LDA}}}{d \rho}\left(\rho_{\mathrm{c}}+\rho_{\Phi}\right)$ to be in $H_{\#}^{q}(\Gamma)$ if $\Phi$ in $\left(H_{\#}^{q}(\Gamma)\right)^{N}$, for $3 / 2<q<m-3 / 2$. We will also establish estimates in negative Sobolev norms, for the proofs of which we will need $\frac{d^{3} e_{\mathrm{Cc}}^{\mathrm{LA}}}{d \rho^{3}}\left(\rho_{\mathrm{c}}+\rho_{\Phi}\right)$ to be in $H_{\#}^{q}(\Gamma)$. Some of our results will therefore be established under the additional assumptions

$$
e_{\mathrm{xc}}^{\mathrm{LDA}} \in C^{n_{m}, \alpha_{m}}((0,+\infty)) \quad \text { where } \quad \begin{aligned}
& n_{m}=[m]+1 \text { and } \alpha_{m}=m-[m]+1 / 2 \text { if } 0 \leq m-[m] \leq 1 / 2, \\
& n_{m}=[m]+2 \text { and } \alpha_{m}=m-[m]-1 / 2 \text { if } 1 / 2<m-[m]<1,
\end{aligned}
$$

(where $[m]$ denotes the integer part of $m$ ) and

$$
e_{\mathrm{xc}}^{\mathrm{LDA}} \in C^{n_{m}, \alpha_{m}}([0,+\infty)) \quad \text { or } \quad \rho_{\mathrm{c}}+\rho^{0}>0 \text { in } \mathbb{R}^{3} .
$$

Recall that for all $q>3 / 2, H_{\#}^{q}(\Gamma)$ is an algebra and that for all $q>3 / 2$, all $g \in C^{[q], q-[q]+\epsilon}(\mathbb{R})$ and all $v \in H_{\#}^{q}(\Gamma), g(v) \in H_{\#}^{q}(\Gamma)$ (see [30]). The condition $e_{\mathrm{xc}}^{\mathrm{LDA}} \in C^{n_{m}, \alpha_{m}}([0,+\infty))$ is not satisfied for usual LDA exchange-correlation functionals. On the other hand, it is of course satisfied for the Hartree (also called reduced Hartree-Fock) model, for which $e_{\mathrm{xc}}^{\mathrm{LDA}}=0$. The condition $\rho_{\mathrm{c}}+\rho^{0}>0$ in $\mathbb{R}^{3}$ seems to be satisfied in practice, but we were not able to establish it rigourously.

By a bootstrap argument on (4.12)-(4.13), we easily infer from (3.19)-(4.14)-(4.15) that $\Phi^{0}$ is actually in $\left(H_{\#}^{m+1 / 2-\epsilon}(\Gamma)\right)^{N}$ for any $\epsilon>0$. 
In fact, (4.1) has an infinity of minimizers since any unitary transform of the Kohn-Sham orbitals $\Phi^{0}$ is also a minimizer of the Kohn-Sham energy. This is a consequence of the following invariance property:

$$
\forall \Phi \in \mathcal{M}, \quad \forall U \in \mathcal{U}(N), \quad U \Phi \in \mathcal{M} \text { and } E^{\mathrm{KS}}(U \Phi)=E^{\mathrm{KS}}(\Phi),
$$

where $\mathcal{U}(N)$ is the group of the real unitary matrices:

$$
\mathcal{U}(N)=\left\{U \in \mathbb{R}^{N \times N} \mid U^{T} U=1_{N}\right\},
$$

$1_{N}$ denoting the identity matrix of rank $N$. This invariance can be exploited to diagonalize the matrix of the Lagrange multipliers of the orthonormality constraints (see e.g. [15]), yielding the existence of a minimizer (still denoted by $\Phi^{0}$ ) with same density $\rho^{0}$, such that

$$
\mathcal{H}_{\rho^{0}}^{\mathrm{KS}} \phi_{i}^{0}=\epsilon_{i}^{0} \phi_{i}^{0},
$$

for some $\epsilon_{1}^{0} \leq \epsilon_{2}^{0} \leq \ldots \leq \epsilon_{N}^{0}$.

Remark 4.1. The Kohn-Sham Hamiltonian $\mathcal{H}_{\rho^{0}}^{\mathrm{KS}}$ is an unbounded self-adjoint operator on $L_{\#}^{2}(\Gamma)$, bounded below, with compact resolvent. Its spectrum therefore is purely discrete. More precisely, it is composed of an increasing sequence of eigenvalues going to infinity, each of these eigenvalues being of finite multiplicity. It is not known whether $\epsilon_{1}^{0}, \ldots, \epsilon_{N}^{0}$ are the lowest eigenvalues (counted with their multiplicities) of $\mathcal{H}_{\rho^{0}}^{\mathrm{KS}}$ (Aufbau principle). However, it seems to be most often (though not always) the case in practice. On the other hand, the Aufbau principle is always satisfied for the extended Kohn-Sham model, for which the first order optimality conditions read

$$
\left\{\begin{array}{l}
\mathcal{H}_{\rho^{0}}^{\mathrm{KS}} \phi_{i}^{0}=\epsilon_{i}^{0} \phi_{i}^{0}, \\
\rho^{0}(x)=2 \sum_{i=1}^{+\infty} n_{i}\left|\phi_{i}^{0}(x)\right|^{2}, \\
\int_{\Gamma} \phi_{i}^{0} \phi_{j}^{0}=\delta_{i j}, \quad 1 \leq i, j<+\infty \\
n_{i}=1 \text { if } \epsilon_{i}^{0}<\epsilon_{\mathrm{F}}, \quad n_{i}=0 \text { if } \epsilon_{i}^{0}>\epsilon_{\mathrm{F}}, \quad 0 \leq n_{i} \leq 1 \text { if } \epsilon_{i}^{0}=\epsilon_{\mathrm{F}}, \quad \sum_{i=1}^{+\infty} n_{i}=N,
\end{array}\right.
$$

where $\epsilon_{\mathrm{F}}$ is the Fermi level (see [8], Sect. 15 for details). In this article, we focus on the standard Kohn-Sham model with integer occupation numbers. We do not need to assume that the Aufbau principle is satisfied, but our analysis requires some coercivity assumption on the second order condition at $\Phi^{0}$ (see (4.20)).

For each $\Phi=\left(\phi_{1}, \ldots, \phi_{N}\right)^{T} \in \mathcal{M}$, we denote by

$$
T_{\Phi} \mathcal{M}=\left\{\left(\psi_{1}, \ldots, \psi_{N}\right)^{T} \in\left(H_{\#}^{1}(\Gamma)\right)^{N} \mid \forall 1 \leq i, j \leq N, \int_{\Gamma} \phi_{i} \psi_{j}+\psi_{i} \phi_{j}=0\right\}
$$

the tangent space to $\mathcal{M}$ at $\Phi$, and we also define

$$
\Phi^{\Perp}=\left\{\Psi=\left(\psi_{1}, \ldots, \psi_{N}\right)^{T} \in\left(H_{\#}^{1}(\Gamma)\right)^{N} \mid \forall 1 \leq i, j \leq N, \int_{\Gamma} \phi_{i} \psi_{j}=0\right\} .
$$

Let us recall (see e.g. Lem. 4 in $[16,29]$ ) that

$$
T_{\Phi} \mathcal{M}=\mathcal{A} \Phi \oplus \Phi^{\Perp}
$$

where $\mathcal{A}=\left\{A \in \mathbb{R}^{N \times N} \mid A^{T}=-A\right\}$ is the space of the $N \times N$ antisymmetric real matrices. 
Since the problem we are considering is a minimization problem, the second order condition further states

$$
\forall W \in T_{\Phi^{0}} \mathcal{M}, \quad a_{\Phi^{0}}(W, W) \geq 0,
$$

where for all $\Psi=\left(\psi_{1}, \ldots, \psi_{N}\right)^{T}$ and $\Upsilon=\left(v_{1}, \ldots, v_{N}\right)^{T}$ in $\left(H_{\#}^{1}(\Gamma)\right)^{N}$,

$$
\begin{aligned}
a_{\Phi^{0}}(\Psi, \Upsilon)= & \frac{1}{4} E^{\mathrm{KS}^{\prime \prime}}\left(\Phi^{0}\right)(\Psi, \Upsilon)-\sum_{i=1}^{N} \epsilon_{i}^{0} \int_{\Gamma} \psi_{i} v_{i} \\
= & \sum_{i=1}^{N}\left\langle\left(\mathcal{H}_{\rho^{0}}^{\mathrm{KS}}-\epsilon_{i}^{0}\right) \psi_{i}, v_{i}\right\rangle_{H_{\#}^{-1}, H_{\#}^{1}}+4 \sum_{i, j=1}^{N} D_{\Gamma}\left(\phi_{i}^{0} \psi_{i}, \phi_{j}^{0} v_{j}\right) \\
& +4 \sum_{i, j=1}^{N} \int_{\Gamma} \frac{d^{2} e_{\mathrm{xc}}^{\mathrm{LDA}}}{d \rho^{2}}\left(\rho_{\mathrm{c}}+\rho^{0}\right) \phi_{i}^{0} \psi_{i} \phi_{j}^{0} v_{j} .
\end{aligned}
$$

It follows from the invariance property (4.16) that

$$
a_{\Phi^{0}}(\Psi, \Psi)=0 \text { for all } \Psi \in \mathcal{A} \Phi^{0} .
$$

This leads us, as in [29], to make the assumption that $a_{\Phi^{0}}$ is positive definite on $\Phi^{0, \Perp}$, so that, as in Proposition 1 in [29], $a_{\Phi^{0}}$ is coercive on $\Phi^{0, \Perp}$ (for the $H_{\#}^{1}$ norm). Thus, in all what follows, we assume that there exists a positive constant $c_{\Phi^{0}}$ such that

$$
\forall \Psi \in \Phi^{0, \Perp}, \quad a_{\Phi^{0}}(\Psi, \Psi) \geq c_{\Phi^{0}}\|\Psi\|_{H_{\#}^{1}}^{2} .
$$

In the linear framework $\left(J=0\right.$ and $E_{\mathrm{xc}}^{\mathrm{LDA}}=0$ in (4.2) $),(4.17)$ and (4.18) respectively read

$$
h \phi_{i}^{0}=\epsilon_{i}^{0} \phi_{i}^{0}
$$

and

$$
a_{\Phi^{0}}(\Psi, \Psi)=\sum_{i=1}^{N}\left\langle\left(h-\epsilon_{i}^{0}\right) \psi_{i}, v_{i}\right\rangle_{H_{\#}^{-1}, H_{\#}^{1}} .
$$

It follows that in the linear framework, the coercivity condition (4.20) is satisfied if and only if (i) $\left(\epsilon_{1}^{0}, \ldots, \epsilon_{N}^{0}\right)$ are the lowest $N$ eigenvalues (including multiplicities) of the linear self-adjoint operator $h=-\frac{1}{2} \Delta+V_{\text {local }}+V_{\mathrm{nl}}$ and (ii) there is a gap $c_{\Phi^{0}}>0$ between the lowest $N^{\text {th }}$ and $(N+1)^{\text {st }}$ eigenvalues of $h$.

Let us now turn to discretization issues. The planewave approximation of (4.1) reads

$$
I_{N_{c}, N_{g}}^{\mathrm{KS}}=\inf \left\{E_{N_{g}}^{\mathrm{KS}}\left(\Phi_{N_{c}}\right), \Phi_{N_{c}} \in V_{N_{c}}^{N} \cap \mathcal{M}\right\}
$$

where

$$
E_{N_{g}}^{\mathrm{KS}}(\Phi)=\sum_{i=1}^{N} \int_{\Gamma}\left|\nabla \phi_{i}\right|^{2}+\int_{\Gamma} \rho_{\Phi} V_{\text {local }}+2 \sum_{i=1}^{N}\left\langle\phi_{i}\left|V_{\mathrm{nl}}\right| \phi_{i}\right\rangle+J\left(\rho_{\Phi}\right)+\int_{\Gamma} \mathcal{I}_{N_{g}}\left(e_{\mathrm{xc}}^{\mathrm{LDA}}\left(\rho_{\mathrm{c}}+\Pi_{2 N_{c}} \rho_{\Phi}\right)\right) .
$$

Here $N_{c}$ is a given positive integer, equal to $\left[\sqrt{2 E_{\mathrm{c}}} L / 2 \pi\right], E_{\mathrm{c}}$ denoting the so-called cut-off energy, and $N_{g} \geq$ $4 N_{c}+1$ is the number of integration points per direction used to evaluate the exchange-correlation contribution. The energy $E_{N_{g}}^{\mathrm{KS}}(\Phi)$ is defined for each $\Phi \in \mathcal{M}$. For $\Phi \in V_{N_{c}}^{N} \cap \mathcal{M}, \Pi_{2 N_{c}} \rho_{\Phi}=\rho_{\Phi}$, so that on this set, $E_{N_{g}}^{\mathrm{KS}}$ differs from $E^{\mathrm{KS}}$ only by the presence of the Fourier interpolation operator $\mathcal{I}_{N_{g}}$ in the exchange-correlation functional. Let us mention that in practice, the terms involving the local and nonlocal components of the pseudopotential are also computed by some interpolation procedure. However, these terms are calculated using spherical harmonics 
and a very fine one dimensional radial grid, so that the resulting integration error is usually much smaller than the interpolation error on the exchange-correlation term. Note that, in addition, the pseudopotential gives rise to linear contributions that can be computed very accurately once and for all (and not at each iteration of the self-consistent field (SCF) algorithm - see [8], Sects. 28-30 for a mathematical introduction to SCF algorithms). We postpone the analysis of (4.21) to a forthcoming article [7], and focus here on the variational approximation

$$
I_{N_{c}}^{\mathrm{KS}}=\inf \left\{E^{\mathrm{KS}}\left(\Phi_{N_{c}}\right), \Phi_{N_{c}} \in V_{N_{c}}^{N} \cap \mathcal{M}\right\}
$$

of (4.1). The unitary invariance of the Kohn-Sham model must be taken into account in the derivation of optimal a priori error estimates. One way to take this invariance into account is to work with one-particle density matrices (see e.g. [8], Sect. 15). The discretized problem is then set on a Grassmann manifold [16]. An alternative is to define for each $\Phi \in \mathcal{M}$ the set

$$
\mathcal{M}^{\Phi}:=\left\{\Psi \in \mathcal{M} \mid\|\Psi-\Phi\|_{L_{\#}^{2}}=\min _{U \in \mathcal{U}(N)}\|U \Psi-\Phi\|_{L_{\#}^{2}}\right\}
$$

and to use the fact that all the local minimizers of (4.23) are obtained by unitary transforms from the local minimizers of

$$
I_{N_{c}}^{\mathrm{KS}}=\inf \left\{E^{\mathrm{KS}}\left(\Phi_{N_{c}}\right), \Phi_{N_{c}} \in V_{N_{c}}^{N} \cap \mathcal{M}^{\Phi^{0}}\right\}
$$

The main result of this section is the following.

Theorem 4.2. Assume that (4.3)-(4.8) hold. Let $\Phi^{0}$ be a local minimizer of (4.1) satisfying (4.20). Then there exist $r^{0}>0$ and $N_{c}^{0}$ such that for $N_{c} \geq N_{c}^{0}$, (4.24) has a unique local minimizer $\Phi_{N_{c}}^{0}$ in the set

$$
\left\{\Phi_{N_{c}} \in V_{N_{c}}^{N} \cap \mathcal{M}^{\Phi^{0}} \mid\left\|\Phi_{N_{c}}-\Phi^{0}\right\|_{H_{\#}^{1}} \leq r^{0}\right\}
$$

Besides,

$$
\begin{aligned}
& \left\|\Phi_{N_{c}}^{0}-\Phi^{0}\right\|_{H_{\#}^{s}} \leq C_{s} N_{c}^{-(2-s)}\left\|\Pi_{N_{c}} \Phi^{0}-\Phi^{0}\right\|_{H_{\#}^{2}}, \\
& \left|\epsilon_{i, N_{c}}^{0}-\epsilon_{i}^{0}\right|_{N_{c} \rightarrow \infty}^{\rightarrow} 0, \\
& \gamma\left\|\Phi_{N_{c}}^{0}-\Phi^{0}\right\|_{H_{\#}^{1}}^{2} \leq I_{N_{c}}^{\mathrm{KS}}-I^{\mathrm{KS}} \leq C\left\|\Phi_{N_{c}}^{0}-\Phi^{0}\right\|_{H_{\#}^{1}}^{2},
\end{aligned}
$$

for all $0 \leq s \leq 2$, and for some constants $\gamma>0, C_{s} \geq 0$, and $C \geq 0$ independent of $N_{c}$, where the $\epsilon_{i, N_{c}}^{0}$ 's are the eigenvalues of the symmetric matrix $\Lambda_{N_{c}}^{0}$, the Lagrange multiplier of the matrix constraint $\int_{\Gamma} \phi_{i, N_{c}} \phi_{j, N_{c}}=\delta_{i j}$.

In addition, if we assume that (4.14) and (4.15) are satisfied, then the following estimates hold:

$$
\begin{aligned}
\left\|\Phi_{N_{c}}^{0}-\Phi^{0}\right\|_{H_{\#}^{s}} & \leq C_{s, \epsilon} N_{c}^{-(m-s+1 / 2-\epsilon)} \\
\left|\epsilon_{i, N_{c}}^{0}-\epsilon_{i}^{0}\right| & \leq C_{\epsilon} N_{c}^{-(2 m-1-\epsilon)}
\end{aligned}
$$

for all $-m+3 / 2<s<m+1 / 2$ and $\epsilon>0$, and for some constants $C_{s, \epsilon} \geq 0$ and $C_{\epsilon} \geq 0$ independent of $N_{c}$.

\subsection{Some technical lemmas}

For $\Phi=\left(\phi_{1}, \ldots, \phi_{N}\right)^{T} \in\left(H_{\#}^{1}(\Gamma)\right)^{N}$ and $\Psi=\left(\psi_{1}, \ldots, \psi_{N}\right)^{T} \in\left(H_{\#}^{1}(\Gamma)\right)^{N}$, we denote by $M_{\Phi, \Psi}$ the $N \times N$ matrix with entries

$$
\left[M_{\Psi, \Phi}\right]_{i j}=\int_{\Gamma} \psi_{i} \phi_{j} .
$$

The following lemma is useful for the analysis of (4.24). We recall that if $A$ and $B$ are symmetric $N \times N$ real matrices, the notation $A \leq B$ means that $x^{T} A x \leq x^{T} B x$ for all $x \in \mathbb{R}^{N}$. 


\section{Lemma 4.3.}

(1) Let $\Phi \in \mathcal{M}$ and $\Psi \in \mathcal{M}$. If $M_{\Psi, \Phi}$ is invertible, then $U_{\Psi, \Phi}=M_{\Psi, \Phi}^{T}\left(M_{\Psi, \Phi} M_{\Psi, \Phi}^{T}\right)^{-1 / 2}$ is the unique minimizer to the problem $\min _{U \in \mathcal{U}(N)}\|U \Psi-\Phi\|_{L_{\#}^{2}}$.

(2) Let $\Phi \in \mathcal{M}$. Then

$$
\mathcal{M}^{\Phi}=\left\{\left(1_{N}-M_{W, W}\right)^{1 / 2} \Phi+W \mid W \in \Phi^{\Perp}, 0 \leq M_{W, W} \leq 1_{N}\right\}
$$

where $1_{N}$ denotes the identity matrix of rank $N$.

(3) Let $\Phi=\left(\phi_{1}, \ldots, \phi_{N}\right)^{T} \in \mathcal{M}$. If $N_{c} \in \mathbb{N}$ is such that

$$
\operatorname{dim}\left(\operatorname{span}\left(\Pi_{N_{c}} \phi_{1}, \ldots, \Pi_{N_{c}} \phi_{N}\right)\right)=N \text {, }
$$

then the unique minimizer of the problem $\min _{\Phi_{N_{c}} \in V_{N_{c}}^{N} \cap \mathcal{M}}\left\|\Phi_{N_{c}}-\Phi\right\|_{L_{\#}^{2}}$ is

$$
\pi_{N_{c}}^{\mathcal{M}} \Phi=\left(M_{\Pi_{N_{c}} \Phi, \Pi_{N_{c}} \Phi}\right)^{-1 / 2} \Pi_{N_{c}} \Phi
$$

In addition, $\pi_{N_{c}}^{\mathcal{M}} \Phi \in V_{N_{c}}^{N} \cap \mathcal{M}^{\Phi}$

$$
\left\|\pi_{N_{c}}^{\mathcal{M}} \Phi-\Phi\right\|_{L_{\#}^{2}} \leq \sqrt{2}\left\|\Pi_{N_{c}} \Phi-\Phi\right\|_{L_{\#}^{2}}
$$

and for all $N_{c}$ large enough,

$$
\left\|\pi_{N_{c}}^{\mathcal{M}} \Phi-\Phi\right\|_{H_{\#}^{1}} \leq\|\Phi\|_{H_{\#}^{1}}\left\|\Pi_{N_{c}} \Phi-\Phi\right\|_{L_{\#}^{2}}^{2}+\left\|\Pi_{N_{c}} \Phi-\Phi\right\|_{H_{\#}^{1}}
$$

(4) Let $N_{c}$ such that $\operatorname{dim}\left(V_{N_{c}}\right) \geq N$ and $\Phi_{N_{c}} \in V_{N_{c}}^{N} \cap \mathcal{M}$. Then

$$
V_{N_{c}}^{N} \cap \mathcal{M}^{\Phi_{N_{c}}}=\left\{\left(1_{N}-M_{W_{N_{c}}, W_{N_{c}}}\right)^{1 / 2} \Phi_{N_{c}}+W_{N_{c}} \mid W_{N_{c}} \in V_{N_{c}}^{N} \cap \Phi_{N_{c}}^{\Perp}, 0 \leq M_{W_{N_{c}}, W_{N_{c}}} \leq 1_{N}\right\}
$$

Proof.

Step 1: proof of the first statement. In order to simplify the notation, we set $M=M_{\Psi, \Phi}$ For each $U \in \mathcal{U}(N)$,

$$
\|U \Psi-\Phi\|_{L_{\#}^{2}}^{2}=2 N-2 \operatorname{Tr}(M U) .
$$

Any critical point $U$ of the problem

$$
\max _{U \in \mathbb{R}^{N \times N} \mid U^{T} U=1_{N}} \operatorname{Tr}(M U)
$$

satisfies an Euler equation of the form $\Lambda U^{T}=M$ for some symmetric matrix $\Lambda$. Besides, $\operatorname{Tr}(M U)=\operatorname{Tr}(\Lambda)$ and $\Lambda^{2}=M M^{T}$. Any maximizer $U$ of (4.33) therefore satisfies $M=\left(M M^{T}\right)^{1 / 2} U^{T}$. Consequently, if $M$ is invertible, the maximizer of (4.33) is unique and reads $U_{\Psi, \Phi}=M^{T}\left(M M^{T}\right)^{-1 / 2}$. It also follows from the definition of the matrix $M$ (recall that $M=M_{\Psi, \Phi}$ ) that $\Psi=M \Phi+W$ with $W \in \Phi^{\Perp}$. Thus,

$$
U_{\Psi, \Phi} \Psi=M^{T}\left(M M^{T}\right)^{-1 / 2} M \Phi+\widetilde{W}
$$

with $\widetilde{W}=U_{\Psi, \Phi} W \in \Phi^{\Perp}$. 
Step 2: proof of the second statement. Each $\Psi \in\left(H_{\#}^{1}(\Gamma)\right)^{N}$ can be written as $\Psi=M \Phi+W$ with $M=M_{\Psi, \Phi} \in$ $\mathbb{R}^{N \times N}$ and $W \in \Phi^{\Perp}$. A simple calculation leads to

$$
\int_{\Gamma} \psi_{i} \psi_{j}=\left[M M^{T}\right]_{i j}+\left[M_{W, W}\right]_{i j}
$$

Hence $\Psi=M \Phi+W \in \mathcal{M}$ if and only if $M M^{T}+M_{W, W}=1_{N}$. In addition, $\Psi \in \mathcal{M}^{\Phi}$ if and only if $\Psi \in \mathcal{M}$ and $U_{\Psi, \Phi}=M^{T}\left(M M^{T}\right)^{-1 / 2}=1_{N}$, that is to say if and only if $M$ is symmetric, $0 \leq M_{W, W} \leq 1_{N}$ and $M=\left(1_{N}-M_{W, W}\right)^{1 / 2}$.

Step 3: proof of (4.30). Let $\left(\chi_{\mu}\right)_{1 \leq \mu \leq \operatorname{dim}\left(V_{N_{c}}\right)}$ be an orthonormal basis of $V_{N_{c}}$ (for the $L_{\#}^{2}$ inner product) and let $\widetilde{C} \in \mathbb{R}^{\operatorname{dim}\left(V_{N_{C}}\right) \times N}$ be the matrix with entries

$$
\widetilde{C}_{\mu, i}=\int_{\Gamma} \phi_{i} \chi_{\mu}
$$

Note that

$$
\Pi_{N_{c}} \phi_{i}=\sum_{\mu=1}^{\operatorname{dim}\left(V_{N_{c}}\right)} \widetilde{C}_{\mu, i} \chi_{\mu} .
$$

For all $\Phi_{N_{c}}=\left(\phi_{N_{c}, 1}, \ldots, \phi_{N_{c}, N}\right)^{T} \in V_{N_{c}}^{N} \cap \mathcal{M}$, each $\phi_{N_{c}, i}$ can be expanded as

$$
\phi_{N_{c}, i}=\sum_{\mu=1}^{\operatorname{dim}\left(V_{N_{c}}\right)} C_{\mu i} \chi_{\mu},
$$

where the matrix $C=\left[C_{\mu i}\right] \in \mathbb{R}^{\operatorname{dim}\left(V_{N_{c}}\right) \times N}$ satisfies the constraint $C^{T} C=1_{N}$. The expansions (4.34) and (4.35) can be recast into the more compact forms

$$
\Pi_{N_{c}} \Phi=\widetilde{C}^{T} \mathcal{X} \quad \text { and } \quad \Phi_{N_{c}}=C^{T} \mathcal{X},
$$

where we have denoted by $\mathcal{X}=\left(\chi_{1}, \ldots, \chi_{\operatorname{dim}\left(V_{N_{c}}\right)}\right)^{T}$. A simple calculation then leads to

$$
\left\|\Phi_{N_{c}}-\Phi\right\|_{L_{\#}^{2}}^{2}=2 N-2 \operatorname{Tr}\left(\widetilde{C}^{T} C\right) .
$$

Reasoning as in Step 1, we obtain that the unique solution to the problem

$$
\max _{C \in \mathbb{R}^{\operatorname{dim}\left(V_{N_{c}}\right) \times N} \mid C^{T} C=1_{N}} \operatorname{Tr}\left(\widetilde{C}^{T} C\right)
$$

is $C=\widetilde{C}\left(\widetilde{C}^{T} \widetilde{C}\right)^{-1 / 2}$. Note that the rank of the matrix $\widetilde{C}$ is $N$ provided that $\operatorname{dim}\left(V_{N_{c}}\right)$ is large enough so that the matrix $\widetilde{C}^{T} \widetilde{C}$ is invertible provided that $\operatorname{dim}\left(V_{N_{c}}\right)$ is large enough. As a consequence, the unique solution to the problem $\min _{\Phi_{N_{c}} \in V_{N_{c}}^{N} \cap \mathcal{M}}\left\|\Phi_{N_{c}}-\Phi\right\|_{L_{\#}^{2}}$ is $\pi_{N_{c}}^{\mathcal{M}} \Phi=\left(\widetilde{C}^{T} \widetilde{C}\right)^{-1 / 2} \widetilde{C}^{T} \mathcal{X}=\left(\widetilde{C}^{T} \widetilde{C}\right)^{-1 / 2} \Pi_{N_{c}} \Phi$. It is then easy to

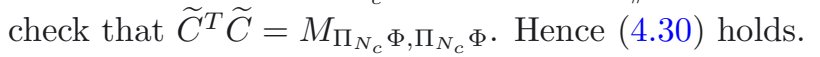

Step 4: proof of the fact that $\pi_{N_{c}}^{\mathcal{M}} \Phi \in \mathcal{M}^{\Phi}$. For all $U \in \mathbb{R}^{N \times N}$ such that $U^{T} U=1_{N}$,

$$
\left\|U \pi_{N_{c}}^{\mathcal{M}} \Phi-\Phi\right\|_{L_{\#}^{2}}^{2}=2\left(N-\operatorname{Tr}\left(U M_{\Pi_{N_{c}} \Phi, \Pi_{N_{c}} \Phi}^{1 / 2}\right)\right),
$$

and the same argument as above leads to the result that this quantity is minimized for

$$
U=M_{\Pi_{N_{c}} \Phi, \Pi_{N_{c}} \Phi}^{1 / 2}\left(M_{\Pi_{N_{c}} \Phi, \Pi_{N_{c}} \Phi}^{1 / 2} M_{\Pi_{N_{c}} \Phi, \Pi_{N_{c}} \Phi}^{1 / 2}\right)^{-1 / 2}=1_{N} .
$$

Therefore, $\pi_{N_{c}}^{\mathcal{M}} \Phi \in \mathcal{M}^{\Phi}$. 
Step 5: proof of (4.31). We infer from (4.36) that

$$
\left\|\pi_{N_{c}}^{\mathcal{M}} \Phi-\Phi\right\|_{L_{\#}^{2}}^{2}=2 N-2 \operatorname{Tr}\left(\left(\widetilde{C}^{T} \widetilde{C}\right)^{1 / 2}\right)=2 \operatorname{Tr}\left(1_{N}-\left(\widetilde{C}^{T} \widetilde{C}\right)^{1 / 2}\right) .
$$

Besides, an easy calculation leads to

$$
\left\|\Pi_{N_{c}} \Phi-\Phi\right\|_{L_{\#}^{2}}^{2}=\operatorname{Tr}\left(1_{N}-\widetilde{C}^{T} \widetilde{C}\right)
$$

Using the fact that

$$
0 \leq\left(1_{N}-\left(\widetilde{C}^{T} \widetilde{C}\right)^{1 / 2}\right) \leq\left(1_{N}-\left(\widetilde{C}^{T} \widetilde{C}\right)^{1 / 2}\right)\left(1_{N}+\left(\widetilde{C}^{T} \widetilde{C}\right)^{1 / 2}\right)=1_{N}-\widetilde{C}^{T} \widetilde{C}
$$

we obtain

$$
\left\|\pi_{N_{c}}^{\mathcal{M}} \Phi-\Phi\right\|_{L_{\#}^{2}}^{2}=2 \operatorname{Tr}\left(1_{N}-\left(\widetilde{C}^{T} \widetilde{C}\right)^{1 / 2}\right) \leq 2 \operatorname{Tr}\left(1_{N}-\widetilde{C}^{T} \widetilde{C}\right)=2\left\|\Pi_{N_{c}} \Phi-\Phi\right\|_{L_{\#}^{2}}^{2} .
$$

Hence (4.31) holds.

Step 6: proof of (4.32). We have

$$
\begin{aligned}
\left\|\pi_{N_{c}}^{\mathcal{M}} \Phi-\Phi\right\|_{H_{\#}^{1}} & \leq\left\|\pi_{N_{c}}^{\mathcal{M}} \Phi-\Pi_{N_{c}} \Phi\right\|_{H_{\#}^{1}}+\left\|\Pi_{N_{c}} \Phi-\Phi\right\|_{H_{\#}^{1}} \\
& =\left\|\left(\left(M_{\Pi_{N_{c}}} \Phi, \Pi_{N_{c}} \Phi\right)^{-1 / 2}-1_{N}\right) \Pi_{N_{c}} \Phi\right\|_{H_{\#}^{1}}+\left\|\Pi_{N_{c}} \Phi-\Phi\right\|_{H_{\#}^{1}} \\
& \leq\left\|\left(M_{\Pi_{N_{c}} \Phi, \Pi_{N_{c}} \Phi}\right)^{-1 / 2}-1_{N}\right\|_{\mathrm{F}}\left\|\Pi_{N_{c}} \Phi\right\|_{H_{\#}^{1}}+\left\|\Pi_{N_{c}} \Phi-\Phi\right\|_{H_{\#}^{1}} \\
& \leq\left\|\left(M_{\Pi_{N_{c}} \Phi, \Pi_{N_{c}} \Phi}\right)^{-1 / 2}-1_{N}\right\|_{\mathrm{F}}\|\Phi\|_{H_{\#}^{1}}+\left\|\Pi_{N_{c}} \Phi-\Phi\right\|_{H_{\#}^{1}},
\end{aligned}
$$

where $\|\cdot\|_{F}$ denotes the Frobenius norm. We then notice that

$$
M_{\Pi_{N_{c}} \Phi, \Pi_{N_{c}} \Phi}=1_{N}-M_{\Pi_{N_{c}} \Phi-\Phi, \Pi_{N_{c}} \Phi-\Phi}
$$

Consequently, for $N_{c}$ large enough,

$$
\left\|\left(M_{\Pi_{N_{c}} \Phi, \Pi_{N_{c}} \Phi}\right)^{-1 / 2}-1_{N}\right\|_{\mathrm{F}} \leq\left\|M_{\Pi_{N_{c}} \Phi-\Phi, \Pi_{N_{c}} \Phi-\Phi}\right\|_{\mathrm{F}} \leq\left\|\Pi_{N_{c}} \Phi-\Phi\right\|_{L_{\#}^{2}}^{2} .
$$

Therefore (4.32) is proved.

Lastly, the fourth assertion easily follows from the second one.

It follows from Lemma 4.3 that for all $\Phi \in \mathcal{M}$, any $\Psi \in \mathcal{M}^{\Phi}$ reads as

$$
\Psi=\Phi+\mathcal{S}(W) \Phi+W
$$

where $W \in \Phi^{\Perp}$ with $0 \leq M_{W, W} \leq 1_{N}$, where and $\mathcal{S}(W)=\left(1_{N}-M_{W, W}\right)^{1 / 2}-1_{N}$ is a $N \times N$ symmetric matrix. Likewise, at the discrete level, for all $\Phi_{N_{c}} \in V_{N_{c}}^{N} \cap \mathcal{M}$, any $\Psi_{N_{c}} \in V_{N_{c}}^{N} \cap \mathcal{M}^{\Phi_{N_{c}}}$ reads as

$$
\Psi_{N_{c}}=\Phi_{N_{c}}+\mathcal{S}\left(W_{N_{c}}\right) \Phi_{N_{c}}+W_{N_{c}}
$$

where $W_{N_{c}} \in V_{N_{c}}^{N} \cap \Phi \frac{\Perp}{N_{c}}$ satisfies $0 \leq M_{W_{N_{c}}, W_{N_{c}}} \leq 1_{N}$. The parametrizations (4.37) and (4.38) of $\mathcal{M}^{\Phi}$ and $V_{N_{c}}^{N} \cap \mathcal{M}^{\Phi_{N_{c}}}$ are going to play essential roles in our analysis. The following lemma collects a few useful properties of the function $W \mapsto \mathcal{S}(W)$. 
Lemma 4.4. Let

$$
K=\left\{W \in\left(L_{\#}^{2}(\Gamma)\right)^{N} \mid 0 \leq M_{W, W} \leq 1_{N}\right\},
$$

and $\mathcal{S}: K \rightarrow \mathbb{R}_{\mathrm{S}}^{N \times N}$ (the space of the symmetric $N \times N$ real matrices) defined by

$$
\mathcal{S}(W)=\left(1_{N}-M_{W, W}\right)^{1 / 2}-1_{N} .
$$

The function $\mathcal{S}$ is continuous on $K$ and differentiable on the interior $\stackrel{\circ}{K}$ of $K$. In addition,

$$
\forall W \in K, \quad\|\mathcal{S}(W)\|_{F} \leq\|W\|_{L_{\#}^{2}}^{2},
$$

and for all $\left(W_{1}, W_{2}, Z\right) \in K \times K \times\left(L_{\#}^{2}(\Gamma)\right)^{N}$ such that $\left\|W_{1}\right\|_{L_{\#}^{2}} \leq \frac{1}{2}$ and $\left\|W_{2}\right\|_{L_{\#}^{2}} \leq \frac{1}{2}$,

$$
\begin{aligned}
\left\|\mathcal{S}\left(W_{1}\right)-\mathcal{S}\left(W_{2}\right)\right\|_{\mathrm{F}} & \leq 2\left(\left\|W_{1}\right\|_{L_{\#}^{2}}+\left\|W_{2}\right\|_{L_{\#}^{2}}\right)\left\|W_{1}-W_{2}\right\|_{L_{\#}^{2}}, \\
\left\|\left(\mathcal{S}^{\prime}\left(W_{1}\right)-\mathcal{S}^{\prime}\left(W_{2}\right)\right) \cdot Z\right\|_{\mathrm{F}} & \leq 4\left\|W_{1}-W_{2}\right\|_{L_{\#}^{2}}\|Z\|_{L_{\#}^{2}}, \\
\|\left(\mathcal{S}^{\prime \prime}\left(W_{1}\right)(Z, Z) \|_{\mathrm{F}}\right. & \leq 4\|Z\|_{L_{\#}^{2}}^{2} .
\end{aligned}
$$

Proof. Diagonalizing $M_{W, W}$ and using the properties of the function $t \mapsto(1-t)^{1 / 2}-1$, we see that $\mathcal{S}$ is continuous on $K$ and differentiable on $\stackrel{\circ}{K}$, and that

$$
\|\mathcal{S}(W)\|_{\mathrm{F}} \leq\left\|M_{W, W}\right\|_{\mathrm{F}} \leq\|W\|_{L_{\#}^{2}}^{2} .
$$

Hence (4.39). As

$$
\mathcal{S}(W)+\frac{1}{2} \mathcal{S}(W)^{2}=-\frac{1}{2} M_{W, W},
$$

we have for all $W \in \stackrel{\circ}{K}$,

$$
\mathcal{S}^{\prime}(W) \cdot Z+\frac{1}{2}\left[\mathcal{S}(W)\left(\mathcal{S}^{\prime}(W) \cdot Z\right)+\left(\mathcal{S}^{\prime}(W) \cdot Z\right) \mathcal{S}(W)\right]=-\frac{1}{2}\left[M_{W, Z}+M_{Z, W}\right] .
$$

Denoting by $A=\mathcal{S}^{\prime}(W) \cdot Z$, we deduce from the above equality that

$$
\|A\|_{\mathrm{F}}^{2}+\operatorname{Tr}\left(A^{2} \mathcal{S}(W)\right) \leq\|A\|_{\mathrm{F}}\left\|M_{W, Z}\right\|_{\mathrm{F}} \leq\|A\|_{\mathrm{F}}\|W\|_{L_{\#}^{2}}\|Z\|_{L_{\#}^{2}} .
$$

As $\left|\operatorname{Tr}\left(A^{2} \mathcal{S}(W)\right)\right| \leq\|A\|_{\mathrm{F}}^{2}\|\mathcal{S}(W)\|_{2} \leq\|A\|_{\mathrm{F}}^{2}\|\mathcal{S}(W)\|_{\mathrm{F}} \leq\|A\|_{\mathrm{F}}^{2}\|W\|_{L_{\#}^{2}}^{2}$, where $\|\mathcal{S}(W)\|_{2}=\sup _{x \in \mathbb{R}^{N} \backslash\{0\}} \frac{|\mathcal{S}(W) x|}{|x|}$ is the matrix norm of $\mathcal{S}(W)$ associated with the Euclidian norm $|\cdot|$ of $\mathbb{R}^{N}$, we finally obtain the inequality

$$
\|A\|_{\mathrm{F}}\left(1-\|W\|_{L_{\#}^{2}}^{2}\right) \leq\|W\|_{L_{\#}^{2}}\|Z\|_{L_{\#}^{2}},
$$

which straightforwardly leads to (4.40) under the conditions $\left\|W_{1}\right\|_{L_{\#}^{2}} \leq \frac{1}{2}$ and $\left\|W_{2}\right\|_{L_{\#}^{2}} \leq \frac{1}{2}$. Lastly,

$$
\begin{aligned}
\left(\mathcal{S}^{\prime}\left(W_{2}\right)-\mathcal{S}^{\prime}\left(W_{1}\right)\right) \cdot Z+\frac{1}{2}\left[\mathcal{S}\left(W_{2}\right)\left(\left(\mathcal{S}^{\prime}\left(W_{2}\right)-\mathcal{S}^{\prime}\left(W_{1}\right)\right) \cdot Z\right)+\left(\left(\mathcal{S}^{\prime}\left(W_{2}\right)-\mathcal{S}^{\prime}\left(W_{1}\right)\right) \cdot Z\right) \mathcal{S}\left(W_{2}\right)\right] \\
\quad+\frac{1}{2}\left[\left(\mathcal{S}^{\prime}\left(W_{1}\right) \cdot Z\right)\left(\mathcal{S}\left(W_{2}\right)-\mathcal{S}\left(W_{1}\right)\right)+\left(\mathcal{S}\left(W_{2}\right)-\mathcal{S}\left(W_{1}\right)\right)\left(\mathcal{S}^{\prime}\left(W_{1}\right) \cdot Z\right)\right]=-\frac{1}{2}\left[M_{W_{2}-W_{1}, Z}+M_{Z, W_{2}-W_{1}}\right]
\end{aligned}
$$


so that still under the conditions $\left\|W_{1}\right\|_{L_{\#}^{2}} \leq \frac{1}{2}$ and $\left\|W_{2}\right\|_{L_{\#}^{2}} \leq \frac{1}{2}$,

$$
\left\|\left(\mathcal{S}^{\prime}\left(W_{2}\right)-\mathcal{S}^{\prime}\left(W_{1}\right)\right) \cdot Z\right\|_{\mathrm{F}} \leq \frac{28}{9}\left\|W_{2}-W_{1}\right\|_{L_{\#}^{2}}\|Z\|_{L_{\#}^{2}} \cdot
$$

Hence (4.41). Lastly, taking $W_{2}=W_{1}+t Z$ in (4.41) and letting $t$ go to zero, we obtain (4.42).

Lemma 4.5. Let $\Phi^{0}$ be a local minimizer of (4.1) satisfying (4.20). Then $a_{\Phi^{0}}$ defines a continuous bilinear form on $\left(H_{\#}^{1}(\Gamma)\right)^{N} \times\left(H_{\#}^{1}(\Gamma)\right)^{N}$, and there exists $N_{c}^{*}$ such that for all $N_{c} \geq N_{c}^{*}$,

$$
\begin{gathered}
\left\|\pi_{N_{c}}^{\mathcal{M}} \Phi^{0}-\Phi^{0}\right\|_{H_{\#}^{1}} \leq 1, \\
a_{\Phi^{0}}\left(\pi_{N_{c}}^{\mathcal{M}} \Phi^{0}-\Phi^{0}, \pi_{N_{c}}^{\mathcal{M}} \Phi^{0}-\Phi^{0}\right) \geq \frac{c_{\Phi^{0}}}{2}\left\|\pi_{N_{c}}^{\mathcal{M}} \Phi^{0}-\Phi^{0}\right\|_{H_{\#}^{1}}^{2}, \\
\forall W \in\left[\pi_{N_{c}}^{\mathcal{M}} \Phi^{0}\right]^{\Perp}, \quad a_{\Phi^{0}}(W, W) \geq \frac{c_{\Phi^{0}}}{2}\|W\|_{H_{\#}^{1}}^{2} .
\end{gathered}
$$

In the sequel, we denote by $C_{\Phi^{0}}$ the continuity constant of $a_{\Phi^{0}}$, i.e.

$$
\forall\left(\Psi, \Psi^{\prime}\right) \in\left(\left(H_{\#}^{1}(\Gamma)\right)^{N}\right)^{2}, \quad\left|a_{\Phi^{0}}\left(\Psi, \Psi^{\prime}\right)\right| \leq C_{\Phi^{0}}\|\Psi\|_{H_{\#}^{1}}\left\|\Psi^{\prime}\right\|_{H_{\#}^{1}} .
$$

Proof. Estimate (4.44) immediately results from the closeness of $\pi_{N_{c}}^{\mathcal{M}} \Phi^{0}$ to $\Phi^{0}$. Using the fact that $\pi_{N_{c}}^{\mathcal{M}} \Phi^{0} \in \mathcal{M}^{\Phi^{0}}$ (see Lem. 4.3, point 3), we get

$$
\pi_{N_{c}}^{\mathcal{M}} \Phi^{0}-\Phi^{0}=\mathcal{S}(W) \Phi^{0}+W
$$

with $W \in\left[\Phi^{0}\right]^{\Perp}$, from which we derive, using (4.39), that

$$
\begin{aligned}
a_{\Phi^{0}}\left(\pi_{N_{c}}^{\mathcal{M}} \Phi^{0}-\Phi^{0}, \pi_{N_{c}}^{\mathcal{M}} \Phi^{0}-\Phi^{0}\right) & =a_{\Phi^{0}}(W, W)+2 a_{\Phi^{0}}\left(W, \mathcal{S}(W) \Phi^{0}\right)+a_{\Phi^{0}}\left(\mathcal{S}(W) \Phi^{0}, \mathcal{S}(W) \Phi^{0}\right) \\
& \geq c_{\Phi^{0}}\|W\|_{H_{\#}^{1}}^{2}-2 C_{\Phi^{0}}\|W\|_{H_{\#}^{1}}\left\|\Phi^{0}\right\|_{H_{\#}^{1}}\|W\|_{L_{\#}^{2}}^{2}-C_{\Phi^{0}}\|W\|_{L_{\#}^{2}}^{4}\left\|\Phi^{0}\right\|_{H_{\#}^{1}}^{2} \\
& \geq\left(c_{\Phi^{0}}-2 C_{\Phi^{0}}\|W\|_{L_{\#}^{2}}\left\|\Phi^{0}\right\|_{H_{\#}^{1}}-C_{\Phi^{0}}\|W\|_{L_{\#}^{2}}^{2}\left\|\Phi^{0}\right\|_{H_{\#}^{1}}^{2}\right)\|W\|_{H_{\#}^{1}}^{2} .
\end{aligned}
$$

As by (4.31), $\left\|\pi_{N_{c}}^{\mathcal{M}} \Phi^{0}-\Phi^{0}\right\|_{L_{\#}^{2}}$ goes to zero when $N_{c}$ goes to infinity, so does $\|W\|_{L_{\#}^{2}}$. Using again (4.39), we deduce from (4.48) that $\|W\|_{H_{\#}^{1} N_{c} \rightarrow \infty}^{\sim}\left\|\pi_{N_{c}}^{\mathcal{M}} \Phi^{0}-\Phi^{0}\right\|_{H_{\#}^{1}}$. Hence (4.45).

Finally, for each $W \in\left[\pi_{N_{c}}^{\mathcal{M}} \Phi^{0}\right]^{\Perp}, W^{\prime}=W-M_{W, \Phi^{0}} \Phi^{0}$ belongs to $\left[\Phi^{0}\right]^{\Perp}$. Remarking that $M_{W, \Phi^{0}}=$ $M_{W, \Phi^{0}-\pi_{N_{c}}^{\mathcal{M}} \Phi^{0}}$, we derive

$$
\left\|M_{W, \Phi^{0}}\right\|_{\mathrm{F}}=\left\|M_{W, \Phi^{0}-\pi_{N_{c}}^{\mathcal{M}_{0}}}\right\|_{\mathrm{F}} \leq \varepsilon\left(N_{c}\right)\|W\|_{L_{\#}^{2}}
$$

where $\varepsilon\left(N_{c}\right)=\left\|\Phi^{0}-\pi_{N_{c}}^{\mathcal{M}} \Phi^{0}\right\|_{L_{\#}^{2}} \rightarrow 0$ when $N_{c}$ goes to infinity. Therefore,

$$
\left\|W-W^{\prime}\right\|_{H_{\#}^{1}} \leq \varepsilon\left(N_{c}\right)\left\|\Phi^{0}\right\|_{H_{\#}^{1}}\|W\|_{H_{\#}^{1}} .
$$

As

we obtain

$$
a_{\Phi^{0}}(W, W)=a_{\Phi^{0}}\left(W^{\prime}, W^{\prime}\right)+2 a_{\Phi^{0}}\left(W^{\prime}, W-W^{\prime}\right)+a_{\Phi^{0}}\left(W-W^{\prime}, W-W^{\prime}\right),
$$

$$
a_{\Phi^{0}}(W, W) \geq c_{\Phi^{0}}\left\|W^{\prime}\right\|_{H_{\#}^{1}}^{2}-2 C_{\Phi^{0}}\left\|W^{\prime}\right\|_{H_{\#}^{1}}\left\|W-W^{\prime}\right\|_{H_{\#}^{1}}-C_{\Phi^{0}}\left\|W-W^{\prime}\right\|_{H_{\#}^{1}}^{2} \geq \frac{c_{\Phi^{0}}}{2}\|W\|_{H_{\#}^{1}}^{2}
$$

for $N_{c}$ large enough. 
Lemma 4.6. There exists $C \geq 0$ such that

- for all $\left(\Upsilon_{1}, \Upsilon_{2}, \Upsilon_{3}\right) \in\left(\left(H_{\#}^{1}(\Gamma)\right)^{N}\right)^{3}$,

$$
\left|\left(E^{\mathrm{KS}^{\prime \prime}}\left(\Phi^{0}+\Upsilon_{1}\right)-E^{\mathrm{KS}}\left(\Phi^{0}\right)\right)\left(\Upsilon_{2}, \Upsilon_{3}\right)\right| \leq C\left(\left\|\Upsilon_{1}\right\|_{H_{\#}^{1}}^{\alpha}+\left\|\Upsilon_{1}\right\|_{H_{\#}^{1}}^{2}\right)\left\|\Upsilon_{2}\right\|_{H_{\#}^{1}}\left\|\Upsilon_{3}\right\|_{H_{\#}^{1}}
$$

- for all $\left(\Upsilon_{1}, \Upsilon_{2}, \Upsilon_{3}\right) \in\left(\left(H_{\#}^{2}(\Gamma)\right)^{N}\right)^{3}$,

$$
\left|\left(E^{\mathrm{KS} \prime \prime}\left(\Phi^{0}+\Upsilon_{1}\right)-E^{\mathrm{KS} \prime \prime}\left(\Phi^{0}\right)\right)\left(\Upsilon_{2}, \Upsilon_{3}\right)\right| \leq C\left(\left\|\Upsilon_{1}\right\|_{L_{\#}^{2}}^{\alpha}+\left\|\Upsilon_{1}\right\|_{L_{\#}^{2}}^{1 / 2}\left\|\Upsilon_{1}\right\|_{H_{\#}^{1}}^{3 / 2}\right)\left\|\Upsilon_{2}\right\|_{L_{\#}^{2}}\left\|\Upsilon_{3}\right\|_{H_{\#}^{2}}
$$

Assume in addition that (4.14) and (4.15) hold. Then, for all $(q, r, s) \in \mathbb{R}_{+}^{3}$ such that $3 / 2<q<m-3 / 2$, $s>3 / 2$ and $r \leq \min (q, s)$, there exist $\eta>0$ and $C \geq 0$ such that

- for all $\left(\Upsilon_{1}, \Upsilon_{2}, \Upsilon_{3}\right) \in\left(H_{\#}^{q}(\Gamma)\right)^{N} \times\left(H_{\#}^{-r}(\Gamma)\right)^{N} \times\left(H_{\#}^{s}(\Gamma)\right)^{N}$ such that $\left\|\Upsilon_{1}\right\|_{H_{\#}^{q}} \leq \eta$,

$$
\left|\left(E^{\mathrm{KS}^{\prime \prime}}\left(\Phi^{0}+\Upsilon_{1}\right)-E^{\mathrm{KS} \prime \prime}\left(\Phi^{0}\right)\right)\left(\Upsilon_{2}, \Upsilon_{3}\right)\right| \leq C\left\|\Upsilon_{1}\right\|_{H_{\#}^{q}}\left\|\Upsilon_{2}\right\|_{H_{\#}^{-r}}\left\|\Upsilon_{3}\right\|_{H_{\#}^{s}}
$$

Proof. Let us define by

$$
r_{\Phi^{0}}\left(\Upsilon_{1}, \Upsilon_{2}, \Upsilon_{3}\right)=\left(E^{\mathrm{KS}^{\prime \prime}}\left(\Phi^{0}+\Upsilon_{1}\right)-E^{\mathrm{KS}{ }^{\prime \prime}}\left(\Phi^{0}\right)\right)\left(\Upsilon_{2}, \Upsilon_{3}\right)
$$

Splitting $r_{\Phi^{0}}\left(\Upsilon_{1}, \Upsilon_{2}, \Upsilon_{3}\right)$ in its Coulomb and exchange-correlation contributions, we obtain

$$
r_{\Phi^{0}}\left(\Upsilon_{1}, \Upsilon_{2}, \Upsilon_{3}\right)=r_{\Phi^{0}}^{\text {Coulomb }}\left(\Upsilon_{1}, \Upsilon_{2}, \Upsilon_{3}\right)+r_{\Phi^{0}}^{\mathrm{xc}}\left(\Upsilon_{1}, \Upsilon_{2}, \Upsilon_{3}\right)
$$

with

$$
\begin{aligned}
r_{\Phi^{0}}^{\text {Coulomb }}\left(\Upsilon_{1}, \Upsilon_{2}, \Upsilon_{3}\right)= & 16 \sum_{i, j=1}^{N}\left(D_{\Gamma}\left(\phi_{i}^{0} v_{1, i}, v_{2, j} v_{3, j}\right)+D_{\Gamma}\left(\phi_{i}^{0} v_{2, i}, v_{1, j} v_{3, j}\right)+D_{\Gamma}\left(\phi_{i}^{0} v_{3, i}, v_{1, j} v_{2, j}\right)\right) \\
& +16 \sum_{i, j=1}^{N} D_{\Gamma}\left(v_{1, i} v_{2, i}, v_{1, j} v_{3, j}\right)+8 \sum_{i, j=1}^{N} D_{\Gamma}\left(v_{1, i}^{2}, v_{2, j} v_{3, j}\right)
\end{aligned}
$$

and

$$
r_{\Phi^{0}}^{\mathrm{xc}}\left(\Upsilon_{1}, \Upsilon_{2}, \Upsilon_{3}\right)=r_{\Phi^{0}}^{\mathrm{xc}, 1}\left(\Upsilon_{1}, \Upsilon_{2}, \Upsilon_{3}\right)+r_{\Phi^{0}}^{\mathrm{xc}, 2}\left(\Upsilon_{1}, \Upsilon_{2}, \Upsilon_{3}\right)
$$

where

$$
\begin{aligned}
r_{\Phi^{0}}^{\mathrm{xc}, 1}\left(\Upsilon_{1}, \Upsilon_{2}, \Upsilon_{3}\right)= & 4 \int_{\Gamma}\left(\frac{d e_{\mathrm{xc}}^{\mathrm{LDA}}}{d \rho}\left(\rho_{\mathrm{c}}+\rho_{\Phi^{0}+\Upsilon_{1}}\right)-\frac{d e_{\mathrm{xc}}^{\mathrm{LDA}}}{d \rho}\left(\rho_{\mathrm{c}}+\rho_{\Phi^{0}}\right)\right)\left(\sum_{i=1}^{N} v_{2, i} v_{3, i}\right) \\
r_{\Phi^{0}}^{\mathrm{xc}, 2}\left(\Upsilon_{1}, \Upsilon_{2}, \Upsilon_{3}\right)= & 16 \int_{\Gamma}\left[\frac{d^{2} e_{\mathrm{xc}}^{\mathrm{LDA}}}{d \rho^{2}}\left(\rho_{\mathrm{c}}+\rho_{\Phi^{0}+\Upsilon_{1}}\right)\left(\sum_{i=1}^{N}\left(\phi_{i}^{0}+v_{1, i}\right) v_{2, i}\right)\left(\sum_{i=1}^{N}\left(\phi_{i}^{0}+v_{1, i}\right) v_{3, i}\right)\right. \\
& \left.-\frac{d^{2} e_{\mathrm{xc}}^{\mathrm{LDA}}}{d \rho^{2}}\left(\rho_{\mathrm{c}}+\rho_{\Phi^{0}}\right)\left(\sum_{i=1}^{N} \phi_{i}^{0} v_{2, i}\right)\left(\sum_{i=1}^{N} \phi_{i}^{0} v_{3, i}\right)\right]
\end{aligned}
$$


Using (3.17), we obtain that there exists a constant $C \geq 0$, such that for all $\left(\Upsilon_{1}, \Upsilon_{2}, \Upsilon_{3}\right) \in\left(\left(H_{\#}^{1}(\Gamma)\right)^{N}\right)^{3}$,

$$
\left|r_{\Phi^{0}}^{\text {Coulomb }}\left(\Upsilon_{1}, \Upsilon_{2}, \Upsilon_{3}\right)\right| \leq C\left(\left\|\Upsilon_{1}\right\|_{L_{\#}^{2}}+\left\|\Upsilon_{1}\right\|_{H_{\#}^{1}}^{2}\right)\left\|\Upsilon_{2}\right\|_{H_{\#}^{1}}\left\|\Upsilon_{3}\right\|_{H_{\#}^{1}}
$$

Let $s_{1} \in \mathbb{R}_{+}$and $s_{3} \geq s_{2} \geq 0$. Using (4.7), we get

$$
\begin{aligned}
\left|\frac{d e_{\mathrm{xc}}^{\mathrm{LDA}}}{d \rho}\left(s_{1}+s_{3}\right)-\frac{d e_{\mathrm{xc}}^{\mathrm{LDA}}}{d \rho}\left(s_{1}+s_{2}\right)\right| & =\left|\int_{0}^{1} \frac{d^{2} e_{\mathrm{xc}}^{\mathrm{LDA}}}{d \rho^{2}}\left(s_{1}+s_{2}+t\left(s_{3}-s_{2}\right)\right)\left(s_{3}-s_{2}\right) \mathrm{d} t\right| \\
& \leq C \int_{0}^{1}\left(1+\left(s_{1}+s_{2}+t\left(s_{3}-s_{2}\right)\right)^{\alpha-1}\right)\left(s_{3}-s_{2}\right) \mathrm{d} t \\
& =C\left[\left(s_{3}-s_{2}\right)+\int_{s_{1}+s_{2}}^{s_{1}+s_{3}} s^{\alpha-1} \mathrm{~d} s\right] \\
& =C\left[\left(s_{3}-s_{2}\right)+\alpha^{-1}\left(\left(s_{1}+s_{3}\right)^{\alpha}-\left(s_{1}+s_{2}\right)^{\alpha}\right)\right] \\
& \leq C\left[\left(s_{3}-s_{2}\right)+\alpha^{-1}\left(s_{3}-s_{2}\right)^{\alpha}\right],
\end{aligned}
$$

by concavity of the function $t \mapsto t^{\alpha}$. Besides,

$$
\left|\rho_{\Phi^{0}+\Upsilon_{1}}-\rho_{\Phi_{0}}\right|=2\left|\sum_{i=1}^{N}\left(2 \phi_{i}^{0} v_{1, i}+v_{1, i}^{2}\right)\right| \leq C\left(\rho_{\Upsilon_{1}}^{1 / 2}+\rho_{\Upsilon_{1}}\right) .
$$

We thus obtain

$$
\left|\frac{d e_{\mathrm{xc}}^{\mathrm{LDA}}}{d \rho}\left(\rho_{\mathrm{c}}+\rho_{\Phi^{0}+\Upsilon_{1}}\right)-\frac{d e_{\mathrm{xc}}^{\mathrm{LDA}}}{d \rho}\left(\rho_{\mathrm{c}}+\rho_{\Phi^{0}}\right)\right| \leq C\left(\left|\rho_{\Phi^{0}+\Upsilon_{1}}-\rho_{\Phi^{0}}\right|+\alpha^{-1}\left|\rho_{\Phi^{0}+\Upsilon_{1}}-\rho_{\Phi^{0}}\right|^{\alpha}\right) \leq C\left[\rho_{\Upsilon_{1}}^{\alpha / 2}+\rho_{\Upsilon_{1}}\right],
$$

from which we infer

$$
\left|r_{\Phi^{0}}^{\mathrm{xc}, 1}\left(\Upsilon_{1}, \Upsilon_{2}, \Upsilon_{3}\right)\right| \leq C \int_{\Gamma}\left(\rho_{\Upsilon_{1}}^{\alpha / 2}+\rho_{\Upsilon_{1}}\right) \rho_{\Upsilon_{2}}^{1 / 2} \rho_{\Upsilon_{3}}^{1 / 2}
$$

Introducing the function

$$
\Phi(t)=\Phi^{0}+t \Upsilon_{1}
$$

we can rewrite $r_{\Phi^{0}}^{\mathrm{xc}, 2}\left(\Upsilon_{1}, \Upsilon_{2}, \Upsilon_{3}\right)$ as

$$
\begin{aligned}
r_{\Phi 0}^{\mathrm{xc}, 2}\left(\Upsilon_{1}, \Upsilon_{2}, \Upsilon_{3}\right)= & 16 \int_{\Gamma}\left[\frac{d^{2} e_{\mathrm{xc}}^{\mathrm{LDA}}}{d \rho^{2}}\left(\rho_{\mathrm{c}}+\rho_{\Phi(1)}\right)\left(\sum_{i=1}^{N} \phi_{i}(1) v_{2, i}\right)\left(\sum_{i=1}^{N} \phi_{i}(1) v_{3, i}\right)\right. \\
& \left.-\frac{d^{2} e_{\mathrm{xc}}^{\mathrm{LDA}}}{d \rho^{2}}\left(\rho_{\mathrm{c}}+\rho_{\Phi(0)}\right)\left(\sum_{i=1}^{N} \phi_{i}(0) v_{2, i}\right)\left(\sum_{i=1}^{N} \phi_{i}(0) v_{3, i}\right)\right] \\
= & 16 \int_{\Gamma} \int_{0}^{1}\left[\frac{d^{2} e_{\mathrm{xc}}^{\mathrm{LDA}}}{d \rho^{2}}\left(\rho_{\mathrm{c}}+\rho_{\Phi(t)}\right)\left(\sum_{i=1}^{N} \phi_{i}(t) v_{2, i}\right)\left(\sum_{i=1}^{N} v_{1, i} v_{3, i}\right)\right. \\
& +\frac{d^{2} e_{\mathrm{xc}}^{\mathrm{LDA}}}{d \rho^{2}}\left(\rho_{\mathrm{c}}+\rho_{\Phi(t)}\right)\left(\sum_{i=1}^{N} v_{1, i} v_{2, i}\right)\left(\sum_{i=1}^{N} \phi_{i}(t) v_{3, i}\right) \\
& \left.+2 \frac{d^{3} e_{\mathrm{xc}}^{\mathrm{LDA}}}{d \rho^{3}}\left(\rho_{\mathrm{c}}+\rho_{\Phi(t)}\right)\left(\sum_{i=1}^{N} \phi_{i}(t) v_{1, i}\right)\left(\sum_{i=1}^{N} \phi_{i}(t) v_{2, i}\right)\left(\sum_{i=1}^{N} \phi_{i}(t) v_{3, i}\right)\right] \mathrm{d} t .
\end{aligned}
$$


Thus, using again (4.7), we obtain

$$
\begin{aligned}
\left|r_{\Phi 0}^{\mathrm{xc}, 2}\left(\Upsilon_{1}, \Upsilon_{2}, \Upsilon_{3}\right)\right| & \leq C \int_{\Gamma} \int_{0}^{1}\left(1+\left(\rho_{\mathrm{c}}+\rho_{\Phi(t)}\right)^{\alpha-1}\right) \rho_{\Phi(t)}^{1 / 2} \rho_{\Upsilon_{1}}^{1 / 2} \rho_{\Upsilon_{2}}^{1 / 2} \rho_{\Upsilon_{3}}^{1 / 2} \mathrm{~d} t \\
& \leq C \int_{\Gamma} \int_{0}^{1}\left(1+\rho_{\Phi(t)}^{\alpha-1}\right) \rho_{\Phi(t)}^{1 / 2} \rho_{\Upsilon_{1}}^{1 / 2} \rho_{\Upsilon_{2}}^{1 / 2} \rho_{\Upsilon_{3}}^{1 / 2} \mathrm{~d} t .
\end{aligned}
$$

Now, for all $0<\alpha \leq 1 / 2$,

$$
\begin{aligned}
\int_{0}^{1} \rho_{\Phi(t)}^{\alpha-1 / 2} \mathrm{~d} t & =2^{\alpha-1 / 2} \int_{0}^{1}\left(\sum_{i=1}^{N} \phi_{i}^{0^{2}}+2 t \sum_{i=1}^{N} \phi_{i}^{0} v_{1, i}+t^{2} \sum_{i=1}^{N} v_{1, i}^{2}\right)^{\alpha-1 / 2} \mathrm{~d} t \\
& =2^{\alpha-1 / 2} \int_{0}^{1}\left(\sum_{i=1}^{N} \phi_{i}^{0^{2}}-\frac{\left(\sum_{i=1}^{N} \phi_{i}^{0} v_{1, i}\right)^{2}}{\sum_{i=1}^{N} v_{1, i}^{2}}+\left(t+\frac{\sum_{i=1}^{N} \phi_{i}^{0} v_{1, i}}{\sum_{i=1}^{N} v_{1, i}^{2}}\right)^{2}\left(\sum_{i=1}^{N} v_{1, i}^{2}\right)\right)^{\alpha-1 / 2} \mathrm{~d} t \\
& \leq 2^{\alpha-1 / 2} \int_{0}^{1}\left|t+\frac{\sum_{i=1}^{N} \phi_{i}^{0} v_{1, i}}{\sum_{i=1}^{N} v_{1, i}^{2}}\right|^{2 \alpha-1}\left(\sum_{i=1}^{N} v_{1, i}^{2}\right)^{\alpha-1 / 2} \mathrm{~d} t \leq \frac{1}{\alpha 2^{\alpha+1 / 2}} \rho_{\Upsilon_{1}}^{\alpha-1 / 2} .
\end{aligned}
$$

As, in addition, $0 \leq \rho_{\Phi(t)} \leq 2\left(\rho_{\Phi^{0}}+t^{2} \rho_{\Upsilon_{1}}\right)$, we obtain, for all $0<\alpha \leq 1$,

$$
\left|r_{\Phi^{0}}^{\mathrm{xc}, 2}\left(\Upsilon_{1}, \Upsilon_{2}, \Upsilon_{3}\right)\right| \leq C \int_{\Gamma}\left(\rho_{\Upsilon_{1}}^{\min (\alpha, 1 / 2)}+\rho_{\Upsilon_{1}}\right) \rho_{\Upsilon_{2}}^{1 / 2} \rho_{\Upsilon_{3}}^{1 / 2}
$$

Collecting (4.53), (4.54) and (4.55), we obtain the first statement using the Sobolev embedding of $H_{\#}^{1}(\Gamma)$ in $L_{\#}^{p}(\Gamma)$ for all $1 \leq p \leq 6$ and Hölder inequality.

In order to obtain the second statement, we use (3.17) and the Sobolev embedding of $H_{\#}^{2}(\Gamma)$ in $L_{\#}^{\infty}(\Gamma)$ to obtain

$$
\left|r_{\Phi^{0}}^{\text {Coulomb }}\left(\Upsilon_{1}, \Upsilon_{2}, \Upsilon_{3}\right)\right| \leq C\left(\left\|\Upsilon_{1}\right\|_{L_{\#}^{2}}+\left\|\Upsilon_{1}\right\|_{L_{\#}^{2}}^{2}\right)\left\|\Upsilon_{2}\right\|_{L_{\#}^{2}}\left\|\Upsilon_{3}\right\|_{H_{\#}^{2}}
$$

Likewise,

$$
\begin{aligned}
\left|r_{\Phi^{0}}^{\mathrm{xc}}\left(\Upsilon_{1}, \Upsilon_{2}, \Upsilon_{3}\right)\right| & \leq C \int_{\Gamma}\left(\rho_{\Upsilon_{1}}^{\alpha / 2}+\rho_{\Upsilon_{1}}\right) \rho_{\Upsilon_{2}}^{1 / 2} \rho_{\Upsilon_{3}}^{1 / 2} \\
& \leq C\left(\left(\int_{\Gamma} \rho_{\Upsilon_{1}}^{\alpha}\right)^{1 / 2}+\left(\int_{\Gamma} \rho_{\Upsilon_{1}}^{2}\right)^{1 / 2}\right)\left\|\Upsilon_{2}\right\|_{L_{\#}^{2}}\left\|\Upsilon_{3}\right\|_{H_{\#}^{2}} \\
& \leq C\left(\left(\int_{\Gamma} \rho_{\Upsilon_{1}}\right)^{\alpha / 2}+\left(\int_{\Gamma} \rho_{\Upsilon_{1}}\right)^{1 / 4}\left(\int_{\Gamma} \rho_{\Upsilon_{1}}^{3}\right)^{1 / 4}\right)\left\|\Upsilon_{2}\right\|_{L_{\#}^{2}}\left\|\Upsilon_{3}\right\|_{H_{\#}^{2}} \\
& \leq C\left(\left\|\Upsilon_{1}\right\|_{L_{\#}^{2}}^{\alpha}+\left\|\Upsilon_{1}\right\|_{L_{\#}^{2}}^{1 / 2}\left\|\Upsilon_{1}\right\|_{H_{\#}^{1}}^{3 / 2}\right)\left\|\Upsilon_{2}\right\|_{L_{\#}^{2}}\left\|\Upsilon_{3}\right\|_{H_{\#}^{2}} .
\end{aligned}
$$

The second statement immediately follows from (4.56) and the above estimate. 
In order to prove the third statement, we first notice that for all $\sigma>3 / 2$ and all $0 \leq r \leq \sigma, H_{\#}^{\sigma}(\Gamma)$ is an ideal of $H_{\#}^{r}(\Gamma)$, which implies, in particular, that for all $r \geq 0$ and all $\sigma>3 / 2$,

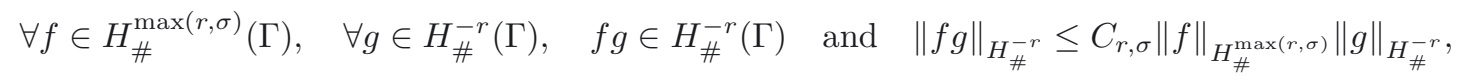

for some constant $C_{r, \sigma} \geq 0$ independent of $f$ and $g$. Using (3.19) and the above property, we obtain that there exists a constant $C_{r, \sigma} \geq 0$ such that

$$
\begin{aligned}
\left|D_{\Gamma}(u v, w z)\right| & \leq\left|\int_{\Gamma} V_{u v}^{\text {Coulomb }} w z\right| \leq\left\|V_{u v}^{\text {Coulomb }}\right\|_{H_{\#}^{r}}\|w z\|_{H_{\#}^{-r}} \\
& \leq C_{r}\|u v\|_{H_{\#}^{r-2}}\|w z\|_{H_{\#}^{-r}} \leq C\|u\|_{H_{\#}^{\max (r-2, \sigma)}}\|v\|_{H_{\#}^{\max (r-2, \sigma)}}\|w\|_{H_{\#}^{\max (r, \sigma)}}\|z\|_{H_{\#}^{-r}},
\end{aligned}
$$

for all $(u, v, w, z) \in H_{\#}^{\max (r-2, \sigma)}(\Gamma) \times H_{\#}^{\max (r-2, \sigma)}(\Gamma) \times H_{\#}^{\max (r, \sigma)}(\Gamma) \times H_{\#}^{-r}(\Gamma)$. It then follows from (4.53) that

$$
\left|r_{\Phi^{0}}^{\text {Coulomb }}\left(\Upsilon_{1}, \Upsilon_{2}, \Upsilon_{3}\right)\right| \leq C\left(\left\|\Upsilon_{1}\right\|_{H_{\#}^{q}}+\left\|\Upsilon_{1}\right\|_{H_{\#}^{q}}^{2}\right)\left\|\Upsilon_{2}\right\|_{H_{\#}^{-r}}\left\|\Upsilon_{3}\right\|_{H_{\#}^{s}}
$$

If (4.14) holds and $e_{\mathrm{xc}}^{\mathrm{LDA}} \in C^{n_{m}, \alpha_{m}}([0,+\infty))$, then for any $\eta>0$, all $\Upsilon_{1} \in\left(H_{\#}^{q}(\Gamma)\right)^{N}$ such that $\left\|\Upsilon_{1}\right\|_{H_{\#}^{q}} \leq \eta$ and all $t \in[0,1]$, the functions $\frac{d^{2} e_{\mathrm{xc}}^{\mathrm{LDA}}}{d \rho^{2}}\left(\rho_{\mathrm{c}}+\rho_{\Phi^{0}+t \Upsilon_{1}}\right)$ and $\frac{d^{3} e_{x c}^{\mathrm{LA}}}{d \rho^{3}}\left(\rho_{\mathrm{c}}+\rho_{\Phi^{0}+t \Upsilon_{1}}\right)$ are bounded in $H_{\#}^{q}(\Gamma)$, uniformly in $\Upsilon_{1}$ and $t$. The same holds if (4.14) is satisfied, if $\rho_{\mathrm{c}}+\rho^{0}>0$ in $\mathbb{R}^{3}$ (which implies that there exists $\beta>0$ such that $\rho_{\mathrm{c}}+\rho^{0} \geq \beta$ since $\rho_{\mathrm{c}}+\rho^{0}$ is continuous and periodic), and if $\left\|\Upsilon_{1}\right\|_{H_{\#}^{q}} \leq \eta$, with $\eta$ small enough to ensure $\rho_{\mathrm{c}}+\rho_{\Phi^{0}+t \Upsilon_{1}} \geq \beta / 2$ for all $t \in[0,1]$. Reasoning as above, we then infer from (4.51), (4.52) and (4.58) that

$$
\left|r_{\Phi^{0}}^{\mathrm{xc}}\left(\Upsilon_{1}, \Upsilon_{2}, \Upsilon_{3}\right)\right| \leq C\left\|\Upsilon_{1}\right\|_{H_{\#}^{q}}\left\|\Upsilon_{2}\right\|_{H_{\#}^{-r}}\left\|\Upsilon_{3}\right\|_{H_{\#}^{s}},
$$

which completes the proof of Lemma 4.6.

Lemma 4.7. Let $\Phi^{0}$ be a local minimizer of (4.1) satisfying (4.20). Then there exists $C \geq 0$ such that for all $\Psi \in \mathcal{M}$,

$$
E^{\mathrm{KS}}(\Psi)=E^{\mathrm{KS}}\left(\Phi^{0}\right)+2 a_{\Phi^{0}}\left(\Psi-\Phi^{0}, \Psi-\Phi^{0}\right)+R\left(\Psi-\Phi^{0}\right),
$$

with

$$
\left|R\left(\Psi-\Phi^{0}\right)\right| \leq C\left(\left\|\Psi-\Phi^{0}\right\|_{H_{\#}^{1}}^{2+\alpha}+\left\|\Psi-\Phi^{0}\right\|_{H_{\#}^{1}}^{4}\right) .
$$

Proof. As the optimality condition (4.17) also reads $\left[E^{\mathrm{KS}^{\prime}}\left(\Phi^{0}\right)\right]_{i}=4 \mathcal{H}_{\rho^{0}}^{\mathrm{KS}} \phi_{i}^{0}=4 \epsilon_{i}^{0} \phi_{i}^{0}$ in $H_{\#}^{-1}(\Gamma)$, we have for all $\Psi \in \mathcal{M}$,

$$
\begin{aligned}
E^{\mathrm{KS}}(\Psi)= & E^{\mathrm{KS}}\left(\Phi^{0}\right)+\left\langle E^{\mathrm{KS}}\left(\Phi^{0}\right), \Psi-\Phi^{0}\right\rangle_{H_{\#}^{-1}, H_{\#}^{1}}+\frac{1}{2} E^{\mathrm{KS}}{ }^{\prime \prime}\left(\Phi^{0}\right)\left(\Psi-\Phi^{0}, \Psi-\Phi^{0}\right) \\
& +\int_{0}^{1}\left(E^{\mathrm{KS} \prime \prime}\left(\Phi^{0}+s\left(\Psi-\Phi^{0}\right)\right)-E^{\mathrm{KS}}\left(\Phi^{0}\right)\right)\left(\Psi-\Phi^{0}, \Psi-\Phi^{0}\right)(1-s) \mathrm{d} s \\
= & E^{\mathrm{KS}}\left(\Phi^{0}\right)+4 \sum_{i=1}^{N} \epsilon_{i}^{0} \int_{\Gamma} \phi_{i}^{0}\left(\psi_{i}-\phi_{i}^{0}\right)+\frac{1}{2} E^{\mathrm{KS}^{\prime \prime}}\left(\Phi^{0}\right)\left(\Psi-\Phi^{0}, \Psi-\Phi^{0}\right) \\
& +\int_{0}^{1}\left(E^{\mathrm{KS}^{\prime \prime}}\left(\Phi^{0}+s\left(\Psi-\Phi^{0}\right)\right)-E^{\mathrm{KS}^{\prime \prime}}\left(\Phi^{0}\right)\right)\left(\Psi-\Phi^{0}, \Psi-\Phi^{0}\right)(1-s) \mathrm{d} s .
\end{aligned}
$$


Using the equality $\left(\phi_{i}^{0}, \psi_{i}-\phi_{i}^{0}\right)_{L_{\#}^{2}}=\left(\phi_{i}^{0}, \psi_{i}\right)_{L_{\#}^{2}}-1=-\frac{1}{2}\left\|\psi_{i}-\phi_{i}^{0}\right\|_{L_{\#}^{2}}$, we obtain

$$
\begin{aligned}
E^{\mathrm{KS}}(\Psi)= & E^{\mathrm{KS}}\left(\Phi^{0}\right)-2 \sum_{i=1}^{N} \epsilon_{i}^{0} \int_{\Gamma}\left(\psi_{i}-\phi_{i}^{0}\right)^{2}+\frac{1}{2} E^{\mathrm{KS}^{\prime \prime}}\left(\Phi^{0}\right)\left(\Psi-\Phi^{0}, \Psi-\Phi^{0}\right) \\
& +\int_{0}^{1}\left(E^{\mathrm{KS} \prime}\left(\Phi^{0}+s\left(\Psi-\Phi^{0}\right)\right)-E^{\mathrm{KS}}{ }^{\prime \prime}\left(\Phi^{0}\right)\right)\left(\Psi-\Phi^{0}, \Psi-\Phi^{0}\right)(1-s) \mathrm{d} s \\
= & E^{\mathrm{KS}}\left(\Phi^{0}\right)+2 a_{\Phi^{0}}\left(\Psi-\Phi^{0}, \Psi-\Phi^{0}\right)+R\left(\Psi-\Phi^{0}\right),
\end{aligned}
$$

where

$$
R(\Upsilon)=\int_{0}^{1}\left(E^{\mathrm{KS}^{\prime \prime}}\left(\Phi^{0}+s \Upsilon\right)-E^{\mathrm{KS} \prime \prime}\left(\Phi^{0}\right)\right)(\Upsilon, \Upsilon)(1-s) \mathrm{d} s
$$

The estimate (4.60) then straightforwardly follows from the first statement of Lemma 4.6.

\subsection{Existence of a discrete solution}

In this subsection, we derive, for $N_{c}$ large enough, the existence of a unique local minimum of the discretized problem (4.24) in the neighborhood of $\pi_{N_{c}}^{\mathcal{M}} \Phi^{0}$.

For this purpose, we use the parametrization (4.38) of the manifold $V_{N_{c}}^{N} \cap \mathcal{M}^{\pi_{N_{c}}^{\mathcal{M}} \Phi^{0}}$ and introduce

$$
\mathcal{B}_{N_{c}}:=\left\{W^{N_{c}} \in V_{N_{c}}^{N} \cap\left[\pi_{N_{c}}^{\mathcal{M}} \Phi^{0}\right]^{\Perp} \mid 0 \leq M_{W^{N_{c}}, W^{N_{c}}} \leq 1\right\},
$$

and $\mathcal{E}_{N_{c}}$ the energy functional defined on $\mathcal{B}_{N_{c}}$ by

$$
\mathcal{E}_{N_{c}}\left(W^{N_{c}}\right)=E^{\mathrm{KS}}\left(\pi_{N_{c}}^{\mathcal{M}} \Phi^{0}+\mathcal{S}\left(W^{N_{c}}\right) \pi_{N_{c}}^{\mathcal{M}} \Phi^{0}+W^{N_{c}}\right)
$$

According to the fourth assertion of Lemma 4.3, the mapping

$$
\begin{aligned}
\mathcal{C}: \mathcal{B}_{N_{c}} & \rightarrow V_{N_{c}}^{N} \cap \mathcal{M}^{\pi_{N_{c}}^{\mathcal{M}} \Phi^{0}} \\
W^{N_{c}} & \mapsto \pi_{N_{c}}^{\mathcal{M}} \Phi^{0}+\mathcal{S}\left(W^{N_{c}}\right) \pi_{N_{c}}^{\mathcal{M}} \Phi^{0}+W^{N_{c}}
\end{aligned}
$$

defines a global map of $V_{N_{c}}^{N} \cap \mathcal{M}^{\pi_{N_{c}}^{\mathcal{M}} \Phi^{0}}$ such that $\mathcal{C}(0)=\pi_{N_{c}}^{\mathcal{M}} \Phi^{0}$. Therefore the minimizers of

$$
\inf \left\{E^{\mathrm{KS}}\left(\Phi_{N_{c}}\right), \Phi_{N_{c}} \in V_{N_{c}}^{N} \cap \mathcal{M}^{\pi_{N_{c}}^{\mathcal{M}} \Phi^{0}}\right\}
$$

are in one-to-one correspondence with those of the minimization problem

$$
\inf \left\{\mathcal{E}_{N_{c}}\left(W^{N_{c}}\right), W^{N_{c}} \in \mathcal{B}_{N_{c}}\right\} .
$$

In a first stage, we prove that for $N_{c}$ large enough, (4.63) has a unique solution in some neighborhood of 0 . As a consequence (4.62) has a unique solution in the vicinity of $\pi_{N_{c}}^{\mathcal{M}} \Phi^{0}$ (for $N_{c}$ large enough). In a second stage, we make use of the unitary invariance (4.16) to prove that for $N_{c}$ large enough, (4.24) has a unique solution in the vicinity of $\Phi^{0}$.

Lemma 4.8. There exist $r>0$ and $N_{c}^{0}$ such that for all $N_{c} \geq N_{c}^{0}$, the functional $\mathcal{E}_{N_{c}}$ has a unique critical point $W_{0}^{N_{c}}$ in the ball

$$
\left\{W^{N_{c}} \in V_{N_{c}}^{N} \cap\left[\pi_{N_{c}}^{\mathcal{M}} \Phi^{0}\right]^{\Perp} \mid\left\|W^{N_{c}}\right\|_{H_{\#}^{1}} \leq r\right\} .
$$


Besides, $W_{0}^{N_{c}}$ is a local minimizer of (4.63) and we have the estimate

$$
\left\|W_{0}^{N_{c}}\right\|_{H_{\#}^{1}} \leq \frac{32 C_{\Phi^{0}}^{3}}{c_{\Phi_{0}}^{3}}\left\|\pi_{N_{c}}^{\mathcal{M}} \Phi^{0}-\Phi^{0}\right\|_{H_{\#}^{1}}
$$

Proof. We infer from Lemma 4.7 that

$$
\begin{aligned}
\mathcal{E}_{N_{c}}\left(W^{N_{c}}\right)= & E^{\mathrm{KS}}\left(\Phi^{0}+\left(\pi_{N_{c}}^{\mathcal{M}} \Phi^{0}-\Phi^{0}\right)+\mathcal{S}\left(W^{N_{c}}\right) \pi_{N_{c}}^{\mathcal{M}} \Phi^{0}+W^{N_{c}}\right) \\
= & E^{\mathrm{KS}}\left(\Phi^{0}\right)+R\left(\left(\pi_{N_{c}}^{\mathcal{M}} \Phi^{0}-\Phi^{0}\right)+\mathcal{S}\left(W^{N_{c}}\right) \pi_{N_{c}}^{\mathcal{M}} \Phi^{0}+W^{N_{c}}\right) \\
& +2 a_{\Phi^{0}}\left(\left(\pi_{N_{c}}^{\mathcal{M}} \Phi^{0}-\Phi^{0}\right)+\mathcal{S}\left(W^{N_{c}}\right) \pi_{N_{c}}^{\mathcal{M}} \Phi^{0}+W^{N_{c}},\left(\pi_{N_{c}}^{\mathcal{M}} \Phi^{0}-\Phi^{0}\right)+\mathcal{S}\left(W^{N_{c}}\right) \pi_{N_{c}}^{\mathcal{M}} \Phi^{0}+W^{N_{c}}\right) \\
= & E^{\mathrm{KS}}\left(\Phi^{0}\right)+2 a_{\Phi^{0}}\left(W^{N_{c}}, W^{N_{c}}\right)+4 a_{\Phi^{0}}\left(W^{N_{c}},\left(\pi_{N_{c}}^{\mathcal{M}} \Phi^{0}-\Phi^{0}\right)\right) \\
& +2 a_{\Phi^{0}}\left(\pi_{N_{c}}^{\mathcal{M}} \Phi^{0}-\Phi^{0}, \pi_{N_{c}}^{\mathcal{M}} \Phi^{0}-\Phi^{0}\right)+\mathcal{R}_{N_{c}}\left(W^{N_{c}}\right),
\end{aligned}
$$

where

$$
\begin{aligned}
\mathcal{R}_{N_{c}}\left(W_{N_{c}}\right)= & 2 a_{\Phi^{0}}\left(\mathcal{S}\left(W^{N_{c}}\right) \pi_{N_{c}}^{\mathcal{M}} \Phi^{0}, \mathcal{S}\left(W^{N_{c}}\right) \pi_{N_{c}}^{\mathcal{M}} \Phi^{0}\right) \\
& +4 a_{\Phi^{0}}\left(\mathcal{S}\left(W^{N_{c}}\right) \pi_{N_{c}}^{\mathcal{M}} \Phi^{0},\left(\pi_{N_{c}}^{\mathcal{M}} \Phi^{0}-\Phi^{0}\right)+W^{N_{c}}\right) \\
& +R\left(\left(\pi_{N_{c}}^{\mathcal{M}} \Phi^{0}-\Phi^{0}\right)+\mathcal{S}\left(W^{N_{c}}\right) \pi_{N_{c}}^{\mathcal{M}} \Phi^{0}+W^{N_{c}}\right) .
\end{aligned}
$$

Thus,

$$
\begin{aligned}
\forall W^{N_{c}} \in \mathcal{B}_{N_{c}}, \quad \mathcal{E}_{N_{c}}\left(W^{N_{c}}\right)= & \mathcal{E}_{N_{c}}(0)+2 a_{\Phi^{0}}\left(W^{N_{c}}, W^{N_{c}}\right)+4 a_{\Phi^{0}}\left(W^{N_{c}},\left(\pi_{N_{c}}^{\mathcal{M}} \Phi^{0}-\Phi^{0}\right)\right) \\
& +\mathcal{R}_{N_{c}}\left(W^{N_{c}}\right)-\mathcal{R}_{N_{c}}(0) .
\end{aligned}
$$

It follows from Lemma $4.7,(4.39)$ and the continuity of $a_{\Phi^{0}}$ on $\left(H_{\#}^{1}(\Gamma)\right)^{N}$ that

$$
\begin{aligned}
\forall W^{N_{c}} \in \mathcal{B}_{N_{c}}, \quad\left|\mathcal{R}_{N_{c}}\left(W^{N_{c}}\right)\right| \leq & C_{\mathcal{R}}\left(\left\|W^{N_{c}}\right\|_{H_{\#}^{1}}^{2+\alpha}+\left\|W^{N_{c}}\right\|_{H_{\#}^{1}}^{8}+\left\|\pi_{N_{c}}^{\mathcal{M}} \Phi^{0}-\Phi^{0}\right\|_{H_{\#}^{1}}^{2+\alpha}\right. \\
& \left.+\left\|\pi_{N_{c}}^{\mathcal{M}} \Phi^{0}-\Phi^{0}\right\|_{H_{\#}^{1}}^{4}+\left\|\pi_{N_{c}}^{\mathcal{M}} \Phi^{0}-\Phi^{0}\right\|_{H_{\#}^{1}}\left\|W^{N_{c}}\right\|_{H_{\#}^{1}}^{2}\right),
\end{aligned}
$$

for a constant $C_{\mathcal{R}} \geq 0$ independent of $N_{c}$. Let us introduce for $N_{c} \geq 0$ and $r>0$ the ball

$$
B_{N_{c}}(r)=\left\{W^{N_{c}} \in V_{N_{c}}^{N} \cap\left[\pi_{N_{c}}^{\mathcal{M}} \Phi^{0}\right]^{\Perp} \mid a_{\Phi^{0}}\left(W^{N_{c}}, W^{N_{c}}\right)<r^{2} a_{\Phi^{0}}\left(\pi_{N_{c}}^{\mathcal{M}} \Phi^{0}-\Phi^{0}, \pi_{N_{c}}^{\mathcal{M}} \Phi^{0}-\Phi^{0}\right)\right\} .
$$

We deduce from Lemma 4.5, that for all $r>0$ and all $N_{c} \geq N_{c}^{*}$, we have

$$
\forall W^{N_{c}} \in \partial B_{N_{c}}(r), \quad \sqrt{\frac{c_{\Phi^{0}}}{2 C_{\Phi^{0}}}} r\left\|\pi_{N_{c}}^{\mathcal{M}} \Phi^{0}-\Phi^{0}\right\|_{H_{\#}^{1}} \leq\left\|W^{N_{c}}\right\|_{H_{\#}^{1}} \leq \sqrt{\frac{2 C_{\Phi^{0}}}{c_{\Phi^{0}}}} r\left\|\pi_{N_{c}}^{\mathcal{M}} \Phi^{0}-\Phi^{0}\right\|_{H_{\#}^{1}} .
$$

Let $r_{0}=2\left(2 C_{\Phi^{0}} / c_{\Phi^{0}}\right)^{5 / 2}$. For all $r>r_{0}$, there exists $N_{c, r} \geq N_{c}^{*}$ such that

$$
\forall N_{c} \geq N_{c, r}, \quad \partial B_{N_{c}}(r) \subset \mathcal{B}_{N_{c}} \quad \text { and } \quad \forall W^{N_{c}} \in \partial B_{N_{c}}(r), \quad\left\|W^{N_{c}}\right\|_{H_{\#}^{1}} \leq 1 .
$$


Therefore, for all $r>r_{0}$ and all $N_{c} \geq N_{c, r}$ we have $\partial B_{N_{c}}(r) \subset \mathcal{B}_{N_{c}}$ and

$$
\begin{aligned}
\forall W^{N_{c}} \in \partial B_{N_{c}}(r), & \mathcal{E}_{N_{c}}(0)+c_{\Phi^{0}}\left\|W^{N_{c}}\right\|_{H_{\#}^{1}}^{2}-4 C_{\Phi^{0}}\left\|W^{N_{c}}\right\|_{H_{\#}^{1}}\left\|\pi_{N_{c}}^{\mathcal{M}} \Phi^{0}-\Phi^{0}\right\|_{H_{\#}^{1}} \\
& -C_{\mathcal{R}}\left(\left\|W^{N_{c}}\right\|_{H_{\#}^{1}}^{2+\alpha}+\left\|W^{N_{c}}\right\|_{H_{\#}^{1}}^{8}+2\left\|\pi_{N_{c}}^{\mathcal{M}} \Phi^{0}-\Phi^{0}\right\|_{H_{\#}^{1}}^{2+\alpha}\right. \\
& \left.+2\left\|\pi_{N_{c}}^{\mathcal{M}} \Phi^{0}-\Phi^{0}\right\|_{H_{\#}^{1}}^{4}+\left\|\pi_{N_{c}}^{\mathcal{M}} \Phi^{0}-\Phi^{0}\right\|_{H_{\#}^{1}}\left\|W^{N_{c}}\right\|_{H_{\#}^{1}}^{2}\right) \\
\geq & \mathcal{E}_{N_{c}}(0)+c_{\Phi^{0}}\left\|W^{N_{c}}\right\|_{H_{\#}^{1}}^{2}-4 C_{\Phi^{0}}\left\|W^{N_{c}}\right\|_{H_{\#}^{1}}\left\|\pi_{N_{c}}^{\mathcal{M}} \Phi^{0}-\Phi^{0}\right\|_{H_{\#}^{1}} \\
& -5 C_{\mathcal{R}}\left(\left\|W^{N_{c}}\right\|_{H_{\#}^{1}}^{2+\alpha}+\left\|\pi_{N_{c}}^{\mathcal{M}} \Phi^{0}-\Phi^{0}\right\|_{H_{\#}^{1}}^{2+\alpha}\right) \\
\geq & \mathcal{E}_{N_{c}}(0)+\frac{c_{\Phi^{0}}^{2}}{2 C_{\Phi^{0}}} r\left(r-r_{0}\right)\left\|\pi_{N_{c}}^{\mathcal{M}} \Phi^{0}-\Phi^{0}\right\|_{H_{\#}^{1}}^{2} \\
& -5 C_{\mathcal{R}}\left(1+\left(\frac{2 C_{\Phi^{0}}}{c_{\Phi^{0}}}\right)^{1+\alpha / 2} r^{2+\alpha}\right)\left\|\pi_{N_{c}}^{\mathcal{M}} \Phi^{0}-\Phi^{0}\right\|_{H_{\#}^{1}}^{2+\alpha} .
\end{aligned}
$$

As $\left\|\pi_{N_{c}}^{\mathcal{M}} \Phi^{0}-\Phi^{0}\right\|_{H_{\#}^{1}}$ goes to zero when $N_{c}$ goes to infinity, we finally obtain that for all $r>r_{0}$, there exists some $N_{c, r}^{\prime} \geq N_{c}^{*}$ such that for all $N_{c} \geq N_{c, r}^{\prime}$,

$$
\partial B_{N_{c}}(r) \subset \mathcal{B}_{N_{c}} \text { and } \forall W^{N_{c}} \in \partial B_{N_{c}}(r), \quad \mathcal{E}_{N_{c}}\left(W^{N_{c}}\right)>\mathcal{E}_{N_{c}}(0)
$$

This proves that for each $N_{c} \geq N_{c, 2 r_{0}}^{\prime}, \mathcal{E}_{N_{c}}$ has a minimizer $W_{0}^{N_{c}}$ in the ball $B_{N_{c}}\left(2 r_{0}\right)$. In particular,

$$
\left\|W_{0}^{N_{c}}\right\|_{H_{\#}^{1}} \leq \frac{32 C_{\Phi^{0}}^{3}}{c_{\Phi_{0}}^{3}}\left\|\pi_{N_{c}}^{\mathcal{M}} \Phi^{0}-\Phi^{0}\right\|_{H_{\#}^{1}}
$$

Let $W_{1}^{N_{c}}$ be a critical point of $\mathcal{E}_{N_{c}}$ such that $\left\|W_{1}^{N_{c}}\right\|_{L_{\#}^{2}} \leq \frac{1}{2}$. We denote by $\delta W^{N_{c}}=W_{1}^{N_{c}}-W_{0}^{N_{c}}$,

$$
\begin{aligned}
& \widetilde{\Phi}_{N_{c}}^{0}=\pi_{N_{c}}^{\mathcal{M}} \Phi^{0}+\mathcal{S}\left(W_{0}^{N_{c}}\right) \pi_{N_{c}}^{\mathcal{M}} \Phi^{0}+W_{0}^{N_{c}}, \\
& \widetilde{\Phi}_{N_{c}}^{1}=\pi_{N_{c}}^{\mathcal{M}} \Phi^{0}+\mathcal{S}\left(W_{1}^{N_{c}}\right) \pi_{N_{c}}^{\mathcal{M}} \Phi^{0}+W_{1}^{N_{c}} .
\end{aligned}
$$

As both $W_{0}^{N_{c}}$ and $W_{1}^{N_{c}}$ are critical points of $\mathcal{E}_{N_{c}}$, we have

$$
\begin{aligned}
& \mathcal{E}_{N_{c}}^{\prime}\left(W_{0}^{N_{c}}\right) \cdot\left(W_{1}^{N_{c}}-W_{0}^{N_{c}}\right)=0, \\
& \mathcal{E}_{N_{c}}^{\prime}\left(W_{1}^{N_{c}}\right) \cdot\left(W_{0}^{N_{c}}-W_{1}^{N_{c}}\right)=0,
\end{aligned}
$$

so that

$$
\left(\mathcal{E}_{N_{c}}^{\prime}\left(W_{1}^{N_{c}}\right)-\mathcal{E}_{N_{c}}^{\prime}\left(W_{0}^{N_{c}}\right)\right) \cdot\left(W_{1}^{N_{c}}-W_{0}^{N_{c}}\right)=0
$$

Using the expression (4.65) for $\mathcal{E}_{N_{c}}$, we can rewrite this equality as

$$
a_{\Phi^{0}}\left(\delta W^{N_{c}}, \delta W^{N_{c}}\right)=b_{\Phi^{0}}^{N_{c}}\left(W_{0}^{N_{c}}, W_{1}^{N_{c}}, \delta W^{N_{c}}\right)+d_{\Phi^{0}}\left(\widetilde{\Phi}_{N_{c}}^{0}, \widetilde{\Phi}_{N_{c}}^{1}, W_{0}^{N_{c}}, W_{1}^{N_{c}}, \delta W^{N_{c}}\right),
$$


where

$$
\begin{aligned}
b_{\Phi^{0}}^{N_{c}}\left(W_{0}^{N_{c}}, W_{1}^{N_{c}}, \delta W^{N_{c}}\right)= & -a_{\Phi^{0}}\left(\left(\mathcal{S}\left(W_{1}^{N_{c}}\right)-\mathcal{S}\left(W_{0}^{N_{c}}\right)\right) \pi_{N_{c}}^{\mathcal{M}} \Phi^{0},\left(\mathcal{S}^{\prime}\left(W_{1}^{N_{c}}\right) \cdot \delta W^{N_{c}}\right) \pi_{N_{c}}^{\mathcal{M}} \Phi^{0}+\delta W^{N_{c}}\right) \\
& -a_{\Phi^{0}}\left(\left(\left(\mathcal{S}^{\prime}\left(W_{1}^{N_{c}}\right)-\mathcal{S}^{\prime}\left(W_{0}^{N_{c}}\right)\right) \cdot \delta W^{N_{c}}\right) \pi_{N_{c}}^{\mathcal{M}} \Phi^{0},\left(\pi_{N_{c}}^{\mathcal{M}} \Phi^{0}-\Phi^{0}\right)\right. \\
& \left.+\mathcal{S}\left(W_{0}^{N_{c}}\right) \pi_{N_{c}}^{\mathcal{M}} \Phi^{0}+W_{0}^{N_{c}}\right)-a_{\Phi^{0}}\left(\left(\mathcal{S}^{\prime}\left(W_{1}^{N_{c}}\right) \cdot \delta W^{N_{c}}\right) \pi_{N_{c}}^{\mathcal{M}} \Phi^{0}, \delta W^{N_{c}}\right)
\end{aligned}
$$

and

$$
\begin{aligned}
d_{\Phi^{0}}\left(\widetilde{\Phi}_{N_{c}}^{0}, \widetilde{\Phi}_{N_{c}}^{1}, W_{0}^{N_{c}}, W_{1}^{N_{c}}, \delta W^{N_{c}}\right)= & \frac{1}{4}\left[R^{\prime}\left(\widetilde{\Phi}_{N_{c}}^{0}-\Phi^{0}\right) \cdot\left(\left(\mathcal{S}^{\prime}\left(W_{0}^{N_{c}}\right) \cdot \delta W^{N_{c}}\right) \pi_{N_{c}}^{\mathcal{M}} \Phi^{0}+\delta W^{N_{c}}\right)\right. \\
& \left.-R^{\prime}\left(\widetilde{\Phi}_{N_{c}}^{1}-\Phi^{0}\right) \cdot\left(\left(\mathcal{S}^{\prime}\left(W_{1}^{N_{c}}\right) \cdot \delta W^{N_{c}}\right) \pi_{N_{c}}^{\mathcal{M}} \Phi^{0}+\delta W^{N_{c}}\right)\right] .
\end{aligned}
$$

Using Lemma 4.4 and (4.66), we obtain that there exists $\widetilde{C}_{\Phi^{0}}$ (depending only on $\Phi^{0}$ ) and $\widetilde{N}_{c}$ such that for all $N_{c} \geq \widetilde{N}_{c}$

$$
\left|b_{\Phi^{0}}^{N_{c}}\left(W_{0}^{N_{c}}, W_{1}^{N_{c}}, \delta W^{N_{c}}\right)\right| \leq \widetilde{C}_{\Phi^{0}}\left(\left\|\pi_{N_{c}}^{\mathcal{M}} \Phi^{0}-\Phi^{0}\right\|_{H_{\#}^{1}}+\left\|W_{1}^{N_{c}}\right\|_{L_{\#}^{2}}\right)\left\|\delta W^{N_{c}}\right\|_{H_{\#}^{1}}^{2} .
$$

On the other hand, remarking that for all $\Psi \in \mathcal{M}$ and all $\delta \Psi \in T_{\Psi} \mathcal{M}$,

$$
R^{\prime}\left(\Psi-\Phi^{0}\right) \cdot \delta \Psi=E^{\mathrm{KS}^{\prime}}(\Psi) \cdot \delta \Psi-4 a_{\Phi^{0}}\left(\Psi-\Phi^{0}, \delta \Psi\right),
$$

and introducing the path $(\Psi(t))_{t \in[0,1]}$, drawn on the manifold $\mathcal{M}$ and connecting $\widetilde{\Phi}_{N_{c}}^{0}$ and $\widetilde{\Phi}_{N_{c}}^{1}$, defined as

$$
\Psi(t)=\Phi^{0}+\mathcal{S}\left(t W_{1}^{N_{c}}+(1-t) W_{0}^{N_{c}}\right) \pi_{N_{c}}^{\mathcal{M}} \Phi^{0}+t W_{1}^{N_{c}}+(1-t) W_{0}^{N_{c}},
$$

we obtain

$$
\begin{aligned}
d_{\Phi^{0}}\left(\widetilde{\Phi}_{N_{c}}^{0}, \widetilde{\Phi}_{N_{c}}^{1}, W_{0}^{N_{c}}, W_{1}^{N_{c}}, \delta W^{N_{c}}\right)= & \frac{1}{4}\left[E^{\mathrm{KS}}(\Psi(0)) \cdot \Psi^{\prime}(0)-E^{\mathrm{KS}}(\Psi(1)) \cdot \Psi^{\prime}(1)\right] \\
& -a_{\Phi^{0}}\left(\Psi(0)-\Phi^{0}, \Psi^{\prime}(0)\right)+a_{\Phi^{0}}\left(\Psi(1)-\Phi^{0}, \Psi^{\prime}(1)\right) \\
= & -\int_{0}^{1}\left[\frac{1}{4} E^{\mathrm{KS}^{\prime \prime}}(\Psi(t))\left(\Psi^{\prime}(t), \Psi^{\prime}(t)\right)+\frac{1}{4} E^{\mathrm{KS}^{\prime}}(\Psi(t)) \cdot \Psi^{\prime \prime}(t)\right. \\
& \left.-a_{\Phi^{0}}\left(\Psi^{\prime}(t), \Psi^{\prime}(t)\right)-a_{\Phi^{0}}\left(\Psi(t)-\Phi^{0}, \Psi^{\prime \prime}(t)\right)\right] \mathrm{d} t .
\end{aligned}
$$

As $\Psi(t)=\left(\psi_{1}(t), \ldots, \psi_{N}(t)\right)^{T} \in \mathcal{M}$ for all $t \in[0,1]$, we have for all $1 \leq i \leq N$ and all $t \in[0,1]$,

$$
\int_{\Gamma} \psi_{i}^{\prime}(t, x)^{2} \mathrm{~d} x=-\int_{\Gamma} \psi_{i}(t, x) \psi_{i}^{\prime \prime}(t, x) \mathrm{d} x
$$

so that

$$
\begin{aligned}
\frac{1}{4} E^{\mathrm{KS}^{\prime}}\left(\Phi^{0}\right) \cdot \Psi^{\prime \prime}(t)-a_{\Phi^{0}}\left(\Psi^{\prime}(t), \Psi^{\prime}(t)\right) & =\sum_{i=1}^{N} \epsilon_{i}^{0} \int_{\Gamma} \phi_{i}^{0} \psi_{i}^{\prime \prime}(t)-\frac{1}{4} E^{\mathrm{KS}}{ }^{\prime \prime}\left(\Phi^{0}\right)\left(\Psi^{\prime}(t), \Psi^{\prime}(t)\right)+\sum_{i=1}^{N} \epsilon_{i}^{0} \int_{\Gamma} \psi_{i}^{\prime}(t)^{2} \\
& =-\sum_{i=1}^{N} \epsilon_{i}^{0} \int_{\Gamma}\left(\psi_{i}(t)-\phi_{i}^{0}\right) \psi_{i}^{\prime \prime}(t)-\frac{1}{4} E^{\mathrm{KS}^{\prime \prime}}\left(\Phi^{0}\right)\left(\Psi^{\prime}(t), \Psi^{\prime}(t)\right) .
\end{aligned}
$$


Consequently,

$$
\begin{aligned}
d_{\Phi^{0}}\left(\widetilde{\Phi}_{N_{c}}^{0}, \widetilde{\Phi}_{N_{c}}^{1}, W_{0}^{N_{c}}, W_{1}^{N_{c}}, \delta W^{N_{c}}\right)= & -\int_{0}^{1}\left[\frac{1}{4}\left(E^{\mathrm{KS}^{\prime \prime}}(\Psi(t))-E^{\mathrm{KS}}{ }^{\prime \prime}\left(\Phi^{0}\right)\right)\left(\Psi^{\prime}(t), \Psi^{\prime}(t)\right)\right. \\
& +\frac{1}{4}\left(E^{\mathrm{KS}^{\prime}}(\Psi(t))-E^{\mathrm{KS}^{\prime}}\left(\Phi^{0}\right)\right) \cdot \Psi^{\prime \prime}(t) \\
& \left.-\sum_{i=1}^{N} \epsilon_{i}^{0} \int_{\Gamma}\left(\psi_{i}(t)-\phi_{i}^{0}\right) \psi_{i}^{\prime \prime}(t)-a_{\Phi^{0}}\left(\Psi(t)-\Phi^{0}, \Psi^{\prime \prime}(t)\right)\right] \mathrm{d} t .
\end{aligned}
$$

Using Lemma 4.6, we obtain

$$
\begin{aligned}
\left|d_{\Phi^{0}}\left(\widetilde{\Phi}_{N_{c}}^{0}, \widetilde{\Phi}_{N_{c}}^{1}, W_{0}^{N_{c}}, W_{1}^{N_{c}}, \delta W^{N_{c}}\right)\right| \leq & C \int_{0}^{1}\left[\left(\left\|\Psi(t)-\Phi^{0}\right\|_{H_{\#}^{1}}^{\alpha}+\left\|\Psi(t)-\Phi^{0}\right\|_{H_{\#}^{1}}^{2}\right)\left\|\Psi^{\prime}(t)\right\|_{H_{\#}^{1}}^{2}\right. \\
& \left.+\left\|\Psi(t)-\Phi^{0}\right\|_{H_{\#}^{1}}\left\|\Psi^{\prime \prime}(t)\right\|_{H_{\#}^{1}}\right] \mathrm{d} t .
\end{aligned}
$$

As

$$
\begin{aligned}
\Psi^{\prime}(t) & =\left(\mathcal{S}^{\prime}\left(t W_{1}^{N_{c}}+(1-t) W_{0}^{N_{c}}\right) \cdot \delta W^{N_{c}}\right) \pi_{N_{c}}^{\mathcal{M}} \Phi^{0}+\delta W^{N_{c}}, \\
\Psi^{\prime \prime}(t) & =\left(\mathcal{S}^{\prime \prime}\left(t W_{1}^{N_{c}}+(1-t) W_{0}^{N_{c}}\right)\left(\delta W^{N_{c}}, \delta W^{N_{c}}\right)\right) \pi_{N_{c}}^{\mathcal{M}} \Phi^{0}
\end{aligned}
$$

we obtain that there exists some constant $C \in \mathbb{R}_{+}$such that for $N_{c}$ large enough,

$$
\left|d_{\Phi^{0}}\left(\widetilde{\Phi}_{N_{c}}^{0}, \widetilde{\Phi}_{N_{c}}^{1}, W_{0}^{N_{c}}, W_{1}^{N_{c}}, \delta W^{N_{c}}\right)\right| \leq C\left(\left\|\pi_{N_{c}}^{\mathcal{M}} \Phi^{0}-\Phi^{0}\right\|_{H_{\#}^{1}}^{\alpha}+\left\|W_{1}^{N_{c}}\right\|_{H_{\#}^{1}}^{\alpha}\right)\left\|\delta W^{N_{c}}\right\|_{H_{\#}^{1}}^{2} .
$$

Thus,

$$
\begin{aligned}
\frac{c_{\Phi^{0}}}{2}\left\|\delta W^{N_{c}}\right\|_{H_{\#}^{1}}^{2} & \leq\left|a_{\Phi^{0}}\left(\delta W^{N_{c}}, \delta W^{N_{c}}\right)\right| \\
& =\left|b_{\Phi^{0}}^{N_{c}}\left(W_{0}^{N_{c}}, W_{1}^{N_{c}}, \delta W^{N_{c}}\right)+d_{\Phi^{0}}\left(\widetilde{\Phi}_{N_{c}}^{0}, \widetilde{\Phi}_{N_{c}}^{1}, W_{0}^{N_{c}}, W_{1}^{N_{c}}, \delta W^{N_{c}}\right)\right| \\
& \leq C\left(\left\|\pi_{N_{c}}^{\mathcal{M}} \Phi^{0}-\Phi^{0}\right\|_{H_{\#}^{1}}^{\alpha}+\left\|W_{1}^{N_{c}}\right\|_{H_{\#}^{1}}^{\alpha}\right)\left\|\delta W^{N_{c}}\right\|_{H_{\#}^{1}}^{2} .
\end{aligned}
$$

This proves that there exists a constant $r>0$ such that for all $N_{c}$ large enough, $\left\|W_{1}^{N_{c}}\right\|_{H_{\#}^{1}} \leq r$ implies $\delta W^{N_{c}}=0$. Hence the result.

As the mapping $B_{N_{c}}\left(2 r_{0}\right) \ni W^{N_{c}} \mapsto \pi_{N_{c}}^{\mathcal{M}} \Phi^{0}+\mathcal{S}\left(W^{N_{c}}\right) \pi_{N_{c}}^{\mathcal{M}} \Phi^{0}+W^{N_{c}}$ defines a local map of $V_{N_{c}}^{N} \cap \mathcal{M}^{\pi_{N_{c}}^{\mathcal{M}} \Phi^{0}}$ in the neighborhood of $\pi_{N_{c}}^{\mathcal{M}} \Phi^{0}$, we obtain that $\widetilde{\Phi}_{N_{c}}^{0}=\pi_{N_{c}}^{\mathcal{M}} \Phi^{0}+\mathcal{S}\left(W_{0}^{N_{c}}\right) \pi_{N_{c}}^{\mathcal{M}} \Phi^{0}+W_{0}^{N_{c}}$ is the unique local minimizer of

$$
\inf \left\{E^{\mathrm{KS}}\left(\Phi_{N_{c}}\right), \Phi_{N_{c}} \in V_{N_{c}}^{N} \cap \mathcal{M}^{\pi_{N_{c}}^{\mathcal{M}} \Phi^{0}}\right\}
$$

in the vicinity of $\pi_{N_{c}}^{\mathcal{M}} \Phi^{0}$. Besides,

$$
\begin{aligned}
\left\|\widetilde{\Phi}_{N_{c}}^{0}-\Phi^{0}\right\|_{H_{\#}^{1}} & \leq\left\|\widetilde{\Phi}_{N_{c}}^{0}-\pi_{N_{c}}^{\mathcal{M}} \Phi^{0}\right\|_{H_{\#}^{1}}+\left\|\pi_{N_{c}}^{\mathcal{M}} \Phi^{0}-\Phi^{0}\right\|_{H_{\#}^{1}} \\
& \leq\left\|S\left(W_{0}^{N_{c}}\right) \pi_{N_{c}}^{\mathcal{M}} \Phi^{0}+W_{0}^{N_{c}}\right\|_{H_{\#}^{1}}+\left\|\pi_{N_{c}}^{\mathcal{M}} \Phi^{0}-\Phi^{0}\right\|_{H_{\#}^{1}} \\
& \leq C\left\|\Pi_{N_{c}} \Phi^{0}-\Phi^{0}\right\|_{H_{\#}^{1}},
\end{aligned}
$$


for a constant $C$ independent of $N_{c}$. We then have

$$
\left\|M_{\widetilde{\Phi}_{N_{c}}^{0}, \Phi^{0}}-1_{N}\right\|_{\mathrm{F}} \leq\left\|\widetilde{\Phi}_{N_{c}}^{0}-\Phi^{0}\right\|_{L_{\#}^{2}} \leq C\left\|\Pi_{N_{c}} \Phi^{0}-\Phi^{0}\right\|_{H_{\#}^{1}}
$$

Let $\Phi_{N_{c}}^{0}=U_{\widetilde{\Phi}_{N_{c}}^{0}, \Phi^{0}} \widetilde{\Phi}_{N_{c}}^{0}$, where $U_{\widetilde{\Phi}_{N_{c}}^{0}, \Phi^{0}}=M_{\widetilde{\Phi}_{N_{c}}^{0}, \Phi^{0}}^{T}\left(M_{\widetilde{\Phi}_{N_{c}}^{0}, \Phi^{0}} M_{\widetilde{\Phi}_{N_{c}}^{0}, \Phi^{0}}^{T}\right)^{-1 / 2}$. Then for each $N_{c} \geq N_{c, 2 r_{0}}^{\prime}, \Phi_{N_{c}}^{0}$ is the unique local minimizer of (4.24) in the set

$$
\left\{\Phi_{N_{c}} \in V_{N_{c}}^{N} \cap \mathcal{M}^{\Phi^{0}} \mid\left\|\Phi_{N_{c}}-\Phi^{0}\right\|_{H_{\#}^{1}} \leq r^{0}\right\}
$$

for some constant $r^{0}>0$ independent of $N_{c}$, and it satisfies

$$
\left\|\Phi_{N_{c}}^{0}-\Phi^{0}\right\|_{H_{\#}^{1}} \leq C\left\|\Pi_{N_{c}} \Phi^{0}-\Phi^{0}\right\|_{H_{\#}^{1}}
$$

for some $C \in \mathbb{R}_{+}$independent of $N_{c}$.

As $\Phi_{N_{c}}^{0} \in \mathcal{M}^{\Phi^{0}}$, we can decompose $\Phi_{N_{c}}^{0}$ as

$$
\Phi_{N_{c}}^{0}=\Phi^{0}+S_{N_{c}}^{0} \Phi^{0}+W_{N_{c}}^{0},
$$

where $S_{N_{c}}^{0}=\mathcal{S}\left(W_{N_{c}}^{0}\right)$ and $W_{N_{c}}^{0} \in \Phi^{0, \Perp}$ (note that $W_{N_{c}}^{0} \notin V_{N_{c}}^{N}$ in general). As by (4.39) and (4.67),

$$
\begin{aligned}
& \left\|S_{N_{c}}^{0}\right\|_{\mathrm{F}} \leq\left\|W_{N_{c}}^{0}\right\|_{L_{\#}^{2}}^{2}, \\
& \left\|S_{N_{c}}^{0}\right\|_{\mathrm{F}}^{2}+\left\|W_{N_{c}}^{0}\right\|_{L_{\#}^{2}}^{2}=\left\|S_{N_{c}}^{0} \Phi^{0}+W_{N_{c}}^{0}\right\|_{L_{\#}^{2}}^{2}=\left\|\Phi_{N_{c}}^{0}-\Phi^{0}\right\|_{L_{\#}^{2}}^{2} \underset{N_{c} \rightarrow \infty}{\longrightarrow} 0,
\end{aligned}
$$

we have, for $N_{c}$ large enough,

$$
\begin{aligned}
& \frac{1}{2}\left\|W_{N_{c}}^{0}\right\|_{L_{\#}^{2}} \leq\left\|\Phi_{N_{c}}^{0}-\Phi^{0}\right\|_{L_{\#}^{2}} \leq 2\left\|W_{N_{c}}^{0}\right\|_{L_{\#}^{2}}, \\
& \frac{1}{2}\left\|W_{N_{c}}^{0}\right\|_{H_{\#}^{1}} \leq\left\|\Phi_{N_{c}}^{0}-\Phi^{0}\right\|_{H_{\#}^{1}} \leq 2\left\|W_{N_{c}}^{0}\right\|_{H_{\#}^{1}} .
\end{aligned}
$$

The discrete solution $\Phi_{N_{c}}^{0}$ satisfies the Euler equations

$$
\forall \Psi_{N_{c}} \in V_{N_{c}}^{N}, \quad\left\langle\mathcal{H}_{\rho_{N_{c}}^{\mathrm{SS}}}^{\mathrm{KS}} \phi_{i, N_{c}}^{0}, \psi_{i, N_{c}}\right\rangle_{H_{\#}^{-1}, H_{\#}^{1}}=\sum_{j=1}^{N}\left[\lambda_{N_{c}}^{0}\right]_{i j}\left(\phi_{j, N_{c}}^{0}, \psi_{j, N_{c}}\right)_{L_{\#}^{2}},
$$

where $\rho_{N_{c}}^{0}=\rho_{\Phi_{N_{c}}^{0}}$ and where the $N \times N$ matrix $\Lambda_{N_{c}}^{0}$ is symmetric (but generally not diagonal). Of course, it follows from the invariance property (4.16) that (4.24) has a local minimizer of the form $U \Phi_{N_{c}}^{0}$ with $U \in \mathcal{U}(N)$ for which the Lagrange multiplier of the orthonormality constraints is a diagonal matrix.

\subsection{A priori error estimates}

We are now in position to derive a priori estimates for $\left\|\Phi_{N_{c}}^{0}-\Phi^{0}\right\|_{H_{\#}^{s}}$ and $\left(\Lambda_{N_{c}}^{0}-\Lambda^{0}\right)$, where we recall that $\Lambda^{0}=\operatorname{diag}\left(\epsilon_{1}^{0}, \ldots, \epsilon_{N}^{0}\right)$. 
Proof of the estimates of the first statement of Theorem 4.2.

Using (2.2), (4.67) and the inverse inequality (3.37), we obtain for each $s \geq 1$ such that $\Phi^{0} \in\left(H_{\#}^{s}(\Gamma)\right)^{N}$ and each $1 \leq r \leq s$

$$
\begin{aligned}
\left\|\Phi_{N_{c}}^{0}-\Phi^{0}\right\|_{H_{\#}^{r}} & \leq\left\|\Phi_{N_{c}}^{0}-\Pi_{N_{c}} \Phi^{0}\right\|_{H_{\#}^{r}}+\left\|\Pi_{N_{c}} \Phi^{0}-\Phi^{0}\right\|_{H_{\#}^{r}} \\
& \leq C N_{c}^{r-1}\left\|\Phi_{N_{c}}^{0}-\Pi_{N_{c}} \Phi^{0}\right\|_{H_{\#}^{1}}+\left\|\Pi_{N_{c}} \Phi^{0}-\Phi^{0}\right\|_{H_{\#}^{r}} \\
& \leq C N_{c}^{r-1}\left(\left\|\Phi_{N_{c}}^{0}-\Phi^{0}\right\|_{H_{\#}^{1}}+\left\|\Phi^{0}-\Pi_{N_{c}} \Phi^{0}\right\|_{H_{\#}^{1}}\right)+\left\|\Pi_{N_{c}} \Phi^{0}-\Phi^{0}\right\|_{H_{\#}^{r}} \\
& \leq C N_{c}^{r-1}\left\|\Pi_{N_{c}} \Phi^{0}-\Phi^{0}\right\|_{H_{\#}^{1}}+\left\|\Pi_{N_{c}} \Phi^{0}-\Phi^{0}\right\|_{H_{\#}^{r}} \\
& \leq C N_{c}^{-(s-r)}\left\|\Pi_{N_{c}} \Phi^{0}-\Phi^{0}\right\|_{H_{\#}^{s}} .
\end{aligned}
$$

In particular, for $s=2$ and $r=3 / 2+\epsilon$ with $0<\epsilon<1 / 2$, we obtain that $\Phi_{N_{c}}^{0}$ converges to $\Phi^{0}$ in $\left(H_{\#}^{3 / 2+\epsilon}(\Gamma)\right)^{N}$, hence in $\left(L_{\#}^{\infty}(\Gamma)\right)^{N}$.

We then proceed as in (3.25) and remark that

$$
\begin{aligned}
\lambda_{i j, N_{c}}^{0}-\lambda_{i j}^{0}= & \left\langle\mathcal{H}_{\rho_{N_{c}}^{0}}^{\mathrm{KS}} \phi_{i, N_{c}}^{0}, \phi_{j, N_{c}}^{0}\right\rangle_{H_{\#}^{-1}, H_{\#}^{1}}-\left\langle\mathcal{H}_{\rho^{0}}^{\mathrm{KS}} \phi_{i}^{0}, \phi_{j}^{0}\right\rangle_{H_{\#}^{-1}, H_{\#}^{1}} \\
= & \left\langle\mathcal{H}_{\rho^{0}}^{\mathrm{KS}}\left(\phi_{i, N_{c}}^{0}-\phi_{i}^{0}\right),\left(\phi_{j, N_{c}}^{0}-\phi_{j}^{0}\right)\right\rangle_{H_{\#}^{-1}, H_{\#}^{1}} \\
& +\epsilon_{i}^{0} \int_{\Gamma} \phi_{i}^{0}\left(\phi_{j, N_{c}}^{0}-\phi_{j}^{0}\right)+\epsilon_{j}^{0} \int_{\Gamma} \phi_{j}^{0}\left(\phi_{i, N_{c}}^{0}-\phi_{i}^{0}\right) \\
& +\int_{\Gamma} V_{\phi_{i, N_{c}}^{0} \phi_{j, N_{c}}^{0}}^{\mathrm{Coulomb}}\left(\rho_{N_{c}}^{0}-\rho^{0}\right) \\
& +\int_{\Gamma}\left(\frac{d e_{\mathrm{xc}}^{\mathrm{LDA}}}{d \rho}\left(\rho_{\mathrm{c}}+\rho_{N_{c}}^{0}\right)-\frac{d e_{\mathrm{xc}}^{\mathrm{LDA}}}{d \rho}\left(\rho_{\mathrm{c}}+\rho^{0}\right)\right) \phi_{i, N_{c}}^{0} \phi_{j, N_{c}}^{0} .
\end{aligned}
$$

As, from (4.68),

$$
\epsilon_{i}^{0} \int_{\Gamma} \phi_{i}^{0}\left(\phi_{j, N_{c}}^{0}-\phi_{j}^{0}\right)+\epsilon_{j}^{0} \int_{\Gamma} \phi_{j}^{0}\left(\phi_{i, N_{c}}^{0}-\phi_{i}^{0}\right)=\left(\epsilon_{i}^{0}+\epsilon_{j}^{0}\right)\left[S_{N_{c}}^{0}\right]_{i j},
$$

we easily obtain, using the convergence of $\Phi_{N_{c}}^{0}$ to $\Phi^{0}$ in $\left(H_{\#}^{1}(\Gamma) \cap L_{\#}^{\infty}(\Gamma)\right)^{N}$,

$$
\left\|\Lambda_{N_{c}}^{0}-\Lambda^{0}\right\|_{\mathrm{F}} \underset{N_{c} \rightarrow \infty}{\longrightarrow} 0
$$

which implies (4.26). For $W \in\left(L_{\#}^{2}(\Gamma)\right)^{N}$, we introduce the adjoint problem

$$
\left\{\begin{array}{l}
\text { find } \Psi_{W} \in \Phi^{0, \Perp} \text { such that } \\
\forall Z \in \Phi^{0, \Perp}, a_{\Phi^{0}}\left(\Psi_{W}, Z\right)=(W, Z)_{L_{\#}^{2}},
\end{array}\right.
$$

the solution of whom exists and is unique by the coercivity assumption (4.20). Clearly,

$$
\left\|\Psi_{W}\right\|_{H_{\#}^{1}} \leq C\|W\|_{L_{\#}^{2}} .
$$

Remarking that $\Psi_{W}$ satisfies

$$
-\Delta \Psi_{W}=L(W)
$$

where $L$ is a bounded linear operator on $L_{\#}^{2}(\Gamma)$, we obtain

$$
\left\|\Psi_{W}\right\|_{H_{\#}^{2}} \leq C\|W\|_{L_{\#}^{2}}
$$


and therefore

$$
\begin{gathered}
\left\|\Psi_{W}-\Pi_{N_{c}} \Psi_{W}\right\|_{L_{\#}^{2}} \leq C N_{c}^{-2}\|W\|_{L_{\#}^{2}}, \\
\left\|\Psi_{W}-\Pi_{N_{c}} \Psi_{W}\right\|_{H_{\#}^{1}} \leq C N_{c}^{-1}\|W\|_{L_{\#}^{2}} .
\end{gathered}
$$

Denoting by $\Psi=\Psi_{\Phi_{N_{c}}^{0}-\Phi^{0}}$ and using (4.68), we get

$$
\begin{aligned}
\left\|\Phi_{N_{c}}^{0}-\Phi^{0}\right\|_{L_{\#}^{2}}^{2}= & \left(\Phi_{N_{c}}^{0}-\Phi^{0}, \Phi_{N_{c}}^{0}-\Phi^{0}\right)_{L_{\#}^{2}} \\
= & \left(\Phi_{N_{c}}^{0}-\Phi^{0}, S_{N_{c}}^{0} \Phi^{0}\right)_{L_{\#}^{2}}+\left(\Phi_{N_{c}}^{0}-\Phi^{0}, W_{N_{c}}^{0}\right)_{L_{\#}^{2}} \\
= & \left(\Phi_{N_{c}}^{0}-\Phi^{0}, S_{N_{c}}^{0} \Phi^{0}\right)_{L_{\#}^{2}}+a_{\Phi^{0}}\left(\Psi, W_{N_{c}}^{0}\right) \\
= & \left(\Phi_{N_{c}}^{0}-\Phi^{0}, S_{N_{c}}^{0} \Phi^{0}\right)_{L_{\#}^{2}}-a_{\Phi^{0}}\left(\Psi, S_{N_{c}}^{0} \Phi^{0}\right)+a_{\Phi^{0}}\left(\Psi, \Phi_{N_{c}}^{0}-\Phi^{0}\right) \\
= & \left(\Phi_{N_{c}}^{0}-\Phi^{0}, S_{N_{c}}^{0} \Phi^{0}\right)_{L_{\#}^{2}}-a_{\Phi^{0}}\left(\Psi, S_{N_{c}}^{0} \Phi^{0}\right)+a_{\Phi^{0}}\left(\Psi-\Pi_{N_{c}} \Psi, \Phi_{N_{c}}^{0}-\Phi^{0}\right) \\
& +a_{\Phi^{0}}\left(\Pi_{N_{c}} \Psi, \Phi_{N_{c}}^{0}-\Phi^{0}\right) .
\end{aligned}
$$

From the definition (4.18), the last term in the above expression reads

$$
a_{\Phi^{0}}\left(\Pi_{N_{c}} \Psi, \Phi_{N_{c}}^{0}-\Phi^{0}\right)=\frac{1}{4} E^{\mathrm{KS}^{\prime \prime}}\left(\Phi^{0}\right)\left(\Pi_{N_{c}} \Psi, \Phi_{N_{c}}^{0}-\Phi^{0}\right)-\sum_{i=1}^{N} \sum_{j=1}^{N} \lambda_{i j}^{0} \int_{\Gamma}\left(\phi_{j, N_{c}}^{0}-\phi_{j}^{0}\right) \Pi_{N_{c}} \psi_{i},
$$

so that from the definition of the continuous and discrete eigenvalue problems

$$
\begin{aligned}
4 a_{\Phi^{0}}\left(\Pi_{N_{c}} \Psi, \Phi_{N_{c}}^{0}-\Phi^{0}\right)= & E^{\mathrm{KS}^{\prime \prime}}\left(\Phi^{0}\right)\left(\Pi_{N_{c}} \Psi, \Phi_{N_{c}}^{0}-\Phi^{0}\right)-E^{\mathrm{KS}^{\prime}}\left(\Phi_{N_{c}}^{0}\right)\left(\Pi_{N_{c}} \Psi\right)+E^{\mathrm{KS}^{\prime}}\left(\Phi^{0}\right)\left(\Pi_{N_{c}} \Psi\right) \\
& +4 \sum_{i=1}^{N} \sum_{j=1}^{N}\left(\lambda_{i j, N_{c}}^{0}-\lambda_{i j}^{0}\right) \int_{\Gamma} \phi_{j, N_{c}}^{0} \Pi_{N_{c}} \psi_{i} .
\end{aligned}
$$

The definition of $\Pi_{N_{c}}$ and the fact that $\Psi \in \Phi^{0, \Perp}$ yields

$$
\int_{\Gamma} \phi_{j, N_{c}}^{0} \Pi_{N_{c}} \psi_{i}=\int_{\Gamma}\left(\phi_{j, N_{c}}^{0}-\phi_{j}^{0}\right) \psi_{i}
$$

which finally provides the estimate

$$
\begin{aligned}
\left\|\Phi_{N_{c}}^{0}-\Phi^{0}\right\|_{L_{\#}^{2}}^{2}= & \left(\Phi_{N_{c}}^{0}-\Phi^{0}, S_{N_{c}}^{0} \Phi^{0}\right)_{L_{\#}^{2}}-a_{\Phi^{0}}\left(\Psi, S_{N_{c}}^{0} \Phi^{0}\right)+a_{\Phi^{0}}\left(\Psi-\Pi_{N_{c}} \Psi, \Phi_{N_{c}}^{0}-\Phi^{0}\right) \\
& -\frac{1}{4}\left(E^{\mathrm{KS}^{\prime}}\left(\Phi_{N_{c}}^{0}\right)\left(\Pi_{N_{c}} \Psi\right)-E^{\mathrm{KS}^{\prime}}\left(\Phi^{0}\right)\left(\Pi_{N_{c}} \Psi\right)-E^{\mathrm{KS}^{\prime \prime}}\left(\Phi^{0}\right)\left(\Phi_{N_{c}}^{0}-\Phi^{0}, \Pi_{N_{c}} \Psi\right)\right) \\
& +\sum_{i=1}^{N} \sum_{j=1}^{N}\left(\lambda_{i j, N_{c}}^{0}-\lambda_{i j}^{0}\right) \int_{\Gamma}\left(\phi_{j, N_{c}}^{0}-\phi_{j}^{0}\right) \psi_{i} .
\end{aligned}
$$

Using the second statement of Lemma 4.6, (4.69), (4.71), (4.77), and (4.79), we infer

$$
\begin{aligned}
\left\|\Phi_{N_{c}}^{0}-\Phi^{0}\right\|_{L_{\#}^{2}} \leq & C\left(\left\|\Phi_{N_{c}}^{0}-\Phi^{0}\right\|_{L_{\#}^{2}}^{2}+N_{c}^{-1}\left\|\Phi_{N_{c}}^{0}-\Phi^{0}\right\|_{H_{\#}^{1}}+\left\|\Phi_{N_{c}}^{0}-\Phi^{0}\right\|_{L_{\#}^{2}}^{1+\alpha}\right. \\
& \left.+\left\|\Phi_{N_{c}}^{0}-\Phi^{0}\right\|_{L_{\#}^{2}}^{3 / 2}\left\|\Phi_{N_{c}}^{0}-\Phi^{0}\right\|_{H_{\#}^{1}}^{3 / 2}+\left\|\Lambda_{N_{c}}^{0}-\Lambda^{0}\right\|_{\mathrm{F}}\left\|\Phi_{N_{c}}^{0}-\Phi^{0}\right\|_{L_{\#}^{2}}\right) .
\end{aligned}
$$


We thus obtain, using (4.67), (4.75) and the above estimate, that asymptotically, when $N_{c}$ goes to infinity,

$$
\left\|\Phi_{N_{c}}^{0}-\Phi^{0}\right\|_{L_{\#}^{2}} \leq C N_{c}^{-1}\left\|\Pi_{N_{c}} \Phi^{0}-\Phi^{0}\right\|_{H_{\#}^{1}} .
$$

Together with (4.73), this straightforwardly leads to (4.25). Finally, (4.27) is an obvious consequence of Lemma 4.7, (4.20), (4.47), and (4.67).

Proof of the second statement of Theorem 4.2.

To proceed further, we need to make an assumption on the regularity of the exchange-correlation potential. In the sequel, we assume that (4.14) and (4.15) hold. As previously mentioned in the proof of Lemma 4.6, assuming that the function $\rho_{\mathrm{c}}+\rho^{0}$ is positive everywhere is equivalent to assuming that there exists a constant $\eta>0$ such that for all $x \in \mathbb{R}^{3}, \rho_{\mathrm{c}}(x)+\rho^{0}(x) \geq \eta$. Besides, we know that the convergence of $\Phi_{N_{c}}^{0}$ to $\Phi^{0}$ holds in $H_{\#}^{2}(\Gamma)$, hence in $L_{\#}^{\infty}(\Gamma)$. As a consequence, for all $x \in \mathbb{R}^{3}$ and all $N_{c}$ large enough, $\rho_{\mathrm{c}}(x)+\rho_{N_{c}}^{0}(x) \geq \eta / 2$.

Using a bootstrap argument on (4.12)-(4.13), we obtain that $\Phi^{0}$ is in $\left(H_{\#}^{m+1 / 2-\epsilon}(\Gamma)\right)^{N}$ for any $\epsilon>0$. Reasoning as in (4.73), we obtain that for each $s \geq 1$ such that $\Phi^{0} \in\left(H_{\#}^{s}(\Gamma)\right)^{N}$ and each $0 \leq r \leq s$, there exists a constant $C$ such that

$$
\left\|\Phi_{N_{c}}^{0}-\Phi^{0}\right\|_{H_{\#}^{r}} \leq C N_{c}^{-(s-r)}\left\|\Pi_{N_{c}} \Phi^{0}-\Phi^{0}\right\|_{H_{\#}^{s}} .
$$

As $\Phi^{0}$ then is in $\left(H_{\#}^{m+1 / 2-\epsilon}(\Gamma)\right)^{N}$ for any $\epsilon>0$, and we deduce from (4.84) that (4.28) holds true for all $0 \leq s<m+1 / 2$.

Then, following the same lines as in the proof of (3.26), we obtain the estimates

$$
\left|\int_{\Gamma} V_{\phi_{i, N_{c}}^{0} \phi_{j, N_{c}}^{0}}^{\text {Coulomb }}\left(\rho_{N_{c}}^{0}-\rho^{0}\right)\right| \leq C\left\|\rho_{N_{c}}^{0}-\rho^{0}\right\|_{H_{\#}^{-r}},
$$

and

$$
\left|\int_{\Gamma}\left(\frac{d e_{\mathrm{xc}}^{\mathrm{LDA}}}{d \rho}\left(\rho_{\mathrm{c}}+\rho^{0}\right)-\frac{d e_{\mathrm{xc}}^{\mathrm{LDA}}}{d \rho}\left(\rho_{\mathrm{c}}+\rho_{N_{c}}^{0}\right)\right) \phi_{i, N_{c}}^{0} \phi_{j, N_{c}}^{0}\right| \leq c\left\|\rho_{N_{c}}^{0}-\rho^{0}\right\|_{H_{\#}^{-r}}
$$

valid for all $0 \leq r<m-3 / 2$. Using these estimates in (4.74), we are lead to

$$
\left|\lambda_{i j, N_{c}}^{0}-\lambda_{i j}^{0}\right| \leq C\left(\left\|\Phi^{0}-\Phi_{N_{c}}^{0}\right\|_{H_{\#}^{1}}^{2}+\left\|\rho_{N_{c}}^{0}-\rho^{0}\right\|_{H_{\#}^{-r}}\right) .
$$

Now,

Noticing that

$$
\left\|\rho_{N_{c}}^{0}-\rho^{0}\right\|_{H_{\#}^{-r}}=\sup _{w \in H_{\#}^{r}(\Gamma)} \frac{\int_{\Gamma}\left(\rho_{N_{c}}^{0}-\rho^{0}\right) w}{\|w\|_{H_{\#}^{r}}} .
$$

$$
\rho_{N_{c}}^{0}-\rho^{0}=\sum_{i=1}^{N}\left|\phi_{i, N_{c}}^{0}\right|^{2}-\sum_{i=1}^{N}\left|\phi_{i}^{0}\right|^{2}=\sum_{i=1}^{N}\left(\phi_{i, N_{c}}^{0}-\phi_{i}^{0}\right)\left(\phi_{i, N_{c}}^{0}+\phi_{i}^{0}\right),
$$

we deduce from (4.58) that

$$
\left\|\rho_{N_{c}}^{0}-\rho^{0}\right\|_{H_{\#}^{-r}} \leq C\left\|\Phi_{N_{c}}^{0}-\Phi^{0}\right\|_{H_{\#}^{-r}},
$$

since $\Phi_{N_{c}}^{0}$ converges, therefore is uniformly bounded in $H_{\#}^{\max (r, 2)}(\Gamma)$. Thus

$$
\left\|\Lambda_{N_{c}}^{0}-\Lambda^{0}\right\|_{\mathrm{F}} \leq C\left(\left\|\Phi_{N_{c}}^{0}-\Phi^{0}\right\|_{H_{\#}^{1}}^{2}+C\left\|\Phi_{N_{c}}^{0}-\Phi^{0}\right\|_{H_{\#}^{-r}}\right) .
$$


The derivation of estimates for $\left\|\Phi_{N_{c}}^{0}-\Phi^{0}\right\|_{H_{\#}^{-r}}$ follows exactly the same lines as the derivation of the $L^{2}$ estimate: starting from the definition

$$
\left\|\Phi_{N_{c}}^{0}-\Phi^{0}\right\|_{H_{\#}^{-r}}=\sup _{W \in\left(H_{\#}^{r}(\Gamma)\right)^{N}} \frac{\left(W, \Phi_{N_{c}}^{0}-\Phi^{0}\right)_{L_{\#}^{2}}}{\|W\|_{H_{\#}^{r}}},
$$

and remarking that the solution $\Psi_{W}$ to the adjoint problem (4.76) satisfies

$$
\left\|\Psi_{W}\right\|_{H_{\#}^{r+2}} \leq C\|W\|_{H_{\#}^{r}},
$$

we proceed as in (4.80)-(4.82) to get

$$
\begin{aligned}
\left(W, \Phi_{N_{c}}^{0}-\Phi^{0}\right)_{L_{\#}^{2}}= & \left(W, S_{N_{c}}^{0} \Phi^{0}\right)_{L_{\#}^{2}}+\left(W, W_{N_{c}}^{0}\right)_{L_{\#}^{2}} \\
= & \left(W, S_{N_{c}}^{0} \Phi^{0}\right)_{L_{\#}^{2}}+a_{\Phi^{0}}\left(\Psi_{W}, W_{N_{c}}^{0}\right) \\
= & \left(W, S_{N_{c}}^{0} \Phi^{0}\right)_{L_{\#}^{2}}-a_{\Phi^{0}}\left(\Psi_{W}, S_{N_{c}}^{0} \Phi^{0}\right)+a_{\Phi^{0}}\left(\Psi_{W}, \Phi_{N_{c}}^{0}-\Phi^{0}\right) \\
= & \left(W, S_{N_{c}}^{0} \Phi^{0}\right)_{L_{\#}^{2}}-a_{\Phi^{0}}\left(\Psi_{W}, S_{N_{c}}^{0} \Phi^{0}\right)+a_{\Phi^{0}}\left(\Psi_{W}-\Pi_{N_{c}} \Psi_{W}, \Phi_{N_{c}}^{0}-\Phi^{0}\right) \\
& -\frac{1}{4}\left(E^{\mathrm{KS}^{\prime}}\left(\Phi_{N_{c}}^{0}\right)\left(\Pi_{N_{c}} \Psi_{W}\right)-E^{\mathrm{KS}^{\prime}}\left(\Phi^{0}\right)\left(\Pi_{N_{c}} \Psi_{W}\right)-E^{\mathrm{KS}^{\prime \prime}}\left(\Phi^{0}\right)\left(\Phi_{N_{c}}^{0}-\Phi^{0}, \Pi_{N_{c}} \Psi_{W}\right)\right) \\
& +\sum_{i=1}^{N} \sum_{j=1}^{N}\left(\lambda_{i j, N_{c}}^{0}-\lambda_{i j}^{0}\right) \int_{\Gamma}\left(\phi_{j, N_{c}}^{0}-\phi_{j}^{0}\right) \psi_{W, i},
\end{aligned}
$$

that yields, using (4.69), (4.71), (4.87), and the third statement of Lemma 4.6 with $q=\max (r, 2)$ and $s=r+2$,

$$
\begin{aligned}
\left\|\Phi_{N_{c}}^{0}-\Phi^{0}\right\|_{H_{\#}^{-r}} \leq & C\left(\left\|\Phi_{N_{c}}^{0}-\Phi^{0}\right\|_{L_{\#}^{2}}^{2}+N_{c}^{-1-r}\left\|\Phi_{N_{c}}^{0}-\Phi^{0}\right\|_{H_{\#}^{1}}+\left\|\Phi_{N_{c}}^{0}-\Phi^{0}\right\|_{H_{\#}^{-r}}\left\|\Phi_{N_{c}}^{0}-\Phi^{0}\right\|_{H_{\#}^{\max (r, 2)}}\right. \\
& \left.+\left\|\Lambda_{N_{c}}^{0}-\Lambda^{0}\right\|_{\mathrm{F}}\left\|\Phi_{N_{c}}^{0}-\Phi^{0}\right\|_{H_{\#}^{-r}}\right)
\end{aligned}
$$

where $\left\|\Phi_{N_{c}}^{0}-\Phi^{0}\right\|_{H_{\#}^{\max (r, 2)}}$ converges to zero thanks to (4.84) since $\max (r, 2)<m+1 / 2$. The proof of (4.28) follows and then we get easily from (4.86) that

$$
\left\|\Lambda_{N_{c}}^{0}-\Lambda^{0}\right\|_{\mathrm{F}} \leq C_{\epsilon} N_{c}^{-(2 m-1-\epsilon)}
$$

Hence (4.29).

Note that the regularity assumption we have made on $e_{\mathrm{xc}}^{\mathrm{LDA}}$ is certainly not optimal. A local regularity in a Sobolev type norm much less stringent than (4.14) should be sufficient to lead to a Hölder type estimate in Lemma 4.6 of the form

$$
\left|\left(E^{\mathrm{KS}^{\prime \prime}}\left(\Phi^{0}+\Upsilon_{1}\right)-E^{\mathrm{KS}}{ }^{\prime \prime}\left(\Phi^{0}\right)\right)\left(\Upsilon_{2}, \Upsilon_{3}\right)\right| \leq C\left\|\Upsilon_{1}\right\|_{H_{\#}^{q}}^{\beta}\left\|\Upsilon_{2}\right\|_{H_{\#}^{-r}}\left\|\Upsilon_{3}\right\|_{H_{\#}^{s}}
$$

for some $\beta>0$, an inequality weaker that (4.49), but sufficient to drive the same rate of convergence for $\left\|\Phi_{N_{c}}^{0}-\Phi^{0}\right\|_{H_{\#}^{-r}}$. Such a Hölder property, which does not seem to be available in the literature, would generalize the Lipschitz property of composition operators acting between Sobolev spaces recently proven in [5]. These considerations go far beyond the purpose of this paper. 

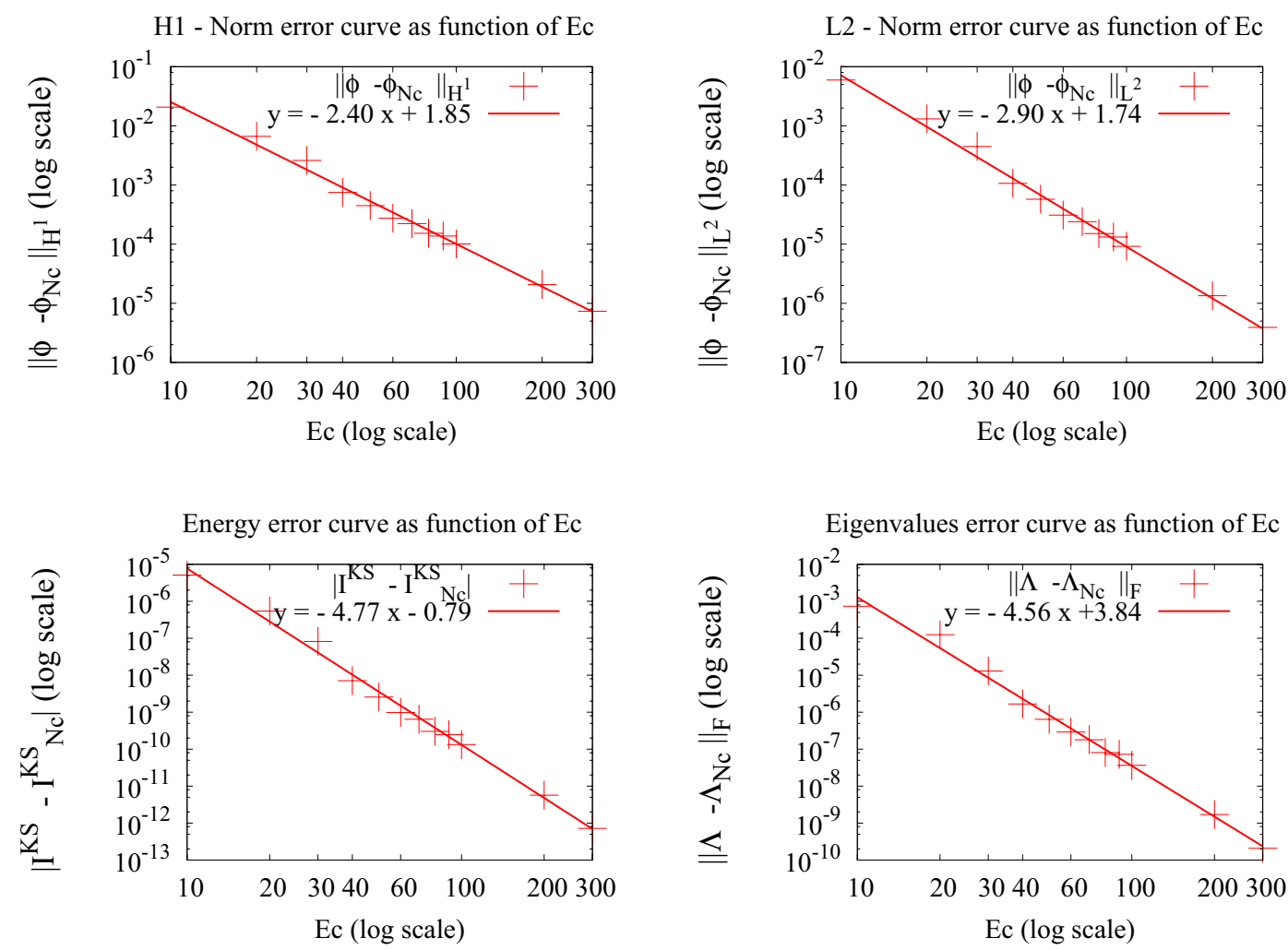

Figure 1. Errors $\left\|\Phi_{N_{c}}^{0}-\Phi^{0}\right\|_{H_{\#}^{1}}$ (up left), $\left\|\Phi_{N_{c}}^{0}-\Phi^{0}\right\|_{L_{\#}^{2}}$ (up right), $\left|I_{N_{c}}^{\mathrm{KS}}-I^{\mathrm{KS}}\right|$ (bottom left) and $\left\|\Lambda^{0}-\Lambda_{N_{c}}^{0}\right\|_{\mathrm{F}}$ (bottom right) as functions of $E_{\mathrm{c}}$ for $\mathrm{H}_{2}$ (in logarithmic scales).

\subsection{Numerical results}

In order to evaluate the quality of the error bounds obtained in Theorem 4.2, we have performed numerical tests using the Abinit software [19] (freely available online, $c f$. http://www.abinit.org), whose main program allows one to find the total energy, charge density and electronic structure of systems (molecules and periodic solids) within DFT, using pseudopotentials and a planewave basis.

We have run simulation tests with the Hartree functional (i.e. with $e_{\mathrm{xc}}^{\mathrm{LDA}}=0$ ), for which there is no numerical integration error. In this particular case, the problems (4.21) (solved by Abinit) and (4.23) (analyzed in Thm. 4.2) are identical.

For Troullier-Martins pseudopotentials, the parameter $m$ in Theorem 4.2 is equal to 5 . Therefore, we expect the following error bounds (as functions of the cut-off energy $E_{\mathrm{c}}=\frac{1}{2}\left(\frac{2 \pi N_{c}}{L}\right)^{2}$ )

$$
\begin{aligned}
\left\|\Phi_{N_{c}}^{0}-\Phi^{0}\right\|_{H_{\#}^{1}} & \leq C_{1, \epsilon} E_{\mathrm{c}}^{-2.25+\epsilon} \\
\left\|\Phi_{N_{c}}^{0}-\Phi^{0}\right\|_{L_{\#}^{2}} & \leq C_{2, \epsilon} E_{\mathrm{c}}^{-2.75+\epsilon} \\
\left|\epsilon_{i, N_{c}}^{0}-\epsilon_{i}^{0}\right| & \leq C_{3, \epsilon} E_{\mathrm{c}}^{-4.5+\epsilon} \\
0 \leq I_{N_{c}}^{\mathrm{KS}}-I^{\mathrm{KS}} & \leq C_{4, \epsilon} E_{\mathrm{c}}^{-4.5+\epsilon}
\end{aligned}
$$

The first tests were performed with the Hydrogen molecule $\left(\mathrm{H}_{2}\right)$. The nuclei were clamped at the points with cartesian coordinates $r_{1}=(-0.7 ; 0 ; 0)$ and $r_{2}=(0.7 ; 0 ; 0)$ (in Bohrs). The simulation cell was a cube of side length $L=10$ Bohrs. The so-obtained numerical errors are plotted in log-scales in Figure 1 . The second series of 

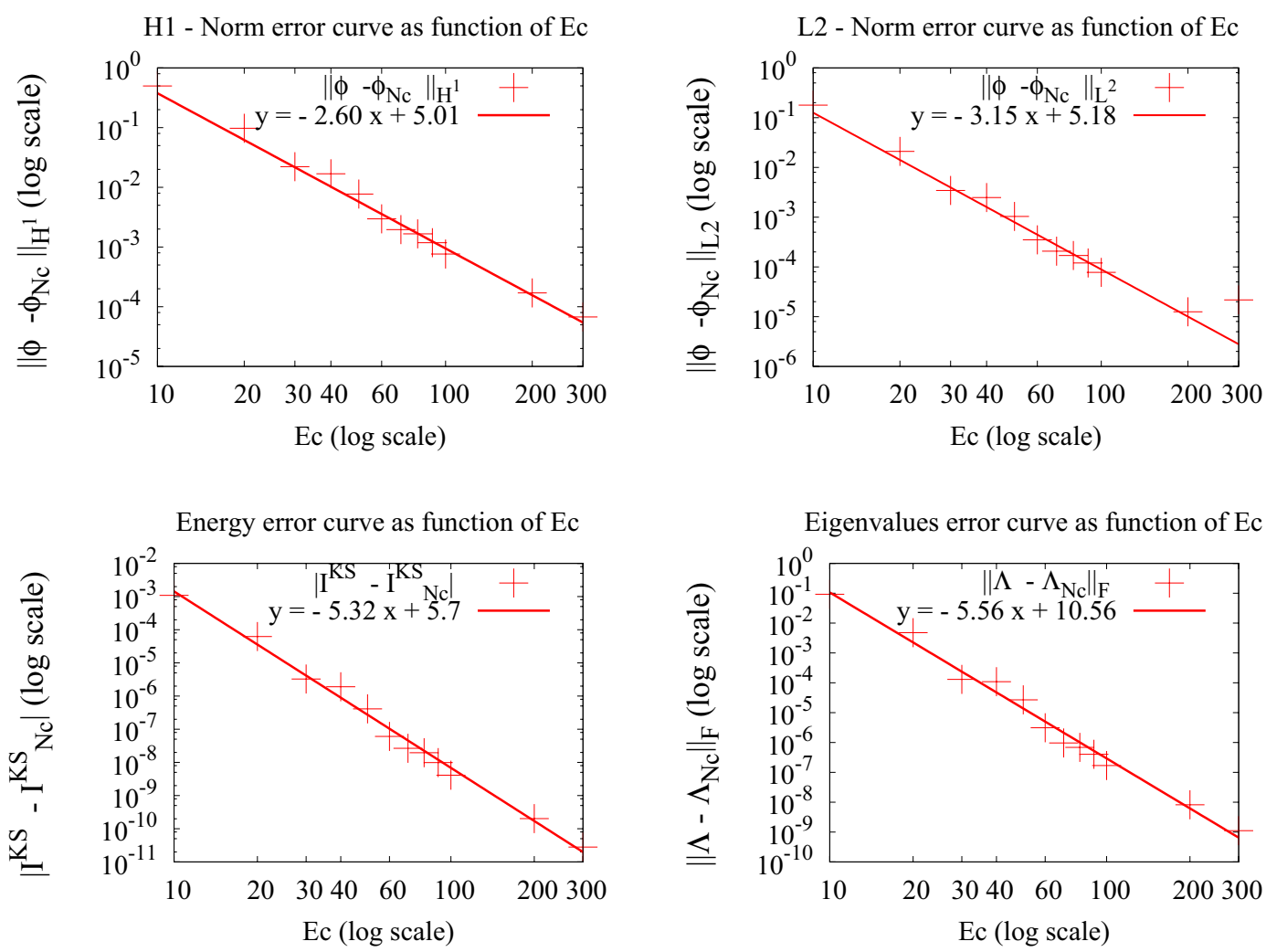

Figure 2. Errors $\left\|\Phi_{N_{c}}^{0}-\Phi^{0}\right\|_{H_{\#}^{1}}$ (up left), $\left\|\Phi_{N_{c}}^{0}-\Phi^{0}\right\|_{L_{\#}^{2}}$ (up right), $\left|I_{N_{c}}^{\mathrm{KS}}-I^{\mathrm{KS}}\right|$ (bottom left) and $\left\|\Lambda^{0}-\Lambda_{N_{c}}^{0}\right\|_{\mathrm{F}}$ (bottom right) as functions of $E_{\mathrm{c}}$ for $\mathrm{N}_{2}$ (in logarithmic scales).

tests were performed with the Nitrogen molecule $\left(\mathrm{N}_{2}\right)$. The nuclei were clamped at positions $r_{1}=(-0.55 ; 0 ; 0)$ and $r_{2}=(0.55 ; 0 ; 0)$ (in Angstroms), and the simulation cell was a cube of side length $L=6$ Angstroms. The numerical errors for $\mathrm{N}_{2}$ are plotted in Figure 2. The reference values for $\Phi^{0}, \epsilon_{i}^{0}$ and $I^{\mathrm{KS}}$ for both $\mathrm{H}_{2}$ and $\mathrm{N}_{2}$ are those obtained for a cut-off energy equal to 500 Hartrees.

These results are in good agreement with the a priori error estimates (4.91)-(4.94) for both the $\mathrm{H}_{2}$ and $\mathrm{N}_{2}$ molecules.

Acknowledgements. This work was done while E.C. was visiting the Division of Applied Mathematics of Brown University, whose support is gratefully acknowledged. This work was also partially supported by the ANR grant LN3M. We are also grateful to V. Ehrlacher, C. Le Bris and the anonymous referees for useful comments and suggestions.

\section{REFERENCES}

[1] A. Anantharaman and E. Cancès, Existence of minimizers for Kohn-Sham models in quantum chemistry. Ann. Inst. Henri Poincaré 26 (2009) 2425-2455.

[2] R. Benguria, H. Brezis and E.H. Lieb, The Thomas-Fermi-von Weizsäcker theory of atoms and molecules. Comm. Math. Phys. 79 (1981) 167-180.

[3] X. Blanc and E. Cancès, Nonlinear instability of density-independent orbital-free kinetic energy functionals. J. Chem. Phys. 122 (2005) 214-106.

[4] M. Born and J.R. Oppenheimer, Zur quantentheorie der molekeln. Ann. Phys. 84 (1927) 457-484.

[5] G. Bourdaud and M. Lanza de Cristoforis, Regularity of the symbolic calculus in Besov algebras. Stud. Math. 184 (2008) 271-298. 
[6] E. Cancès, R. Chakir and Y. Maday, Numerical analysis of nonlinear eigenvalue problems. J. Sci. Comput. 45 (2010) $90-117$.

[7] E. Cancès, R. Chakir, V. Ehrlacher and Y. Maday, in preparation.

[8] E. Cancès, M. Defranceschi, W. Kutzelnigg, C. Le Bris and Y. Maday, Computational quantum chemistry: a primer, in Handbook of numerical analysis X. North-Holland, Amsterdam (2003) 3-270.

[9] E. Cancès, C. Le Bris and Y. Maday, Méthodes mathématiques en chimie quantique. Springer (2006).

[10] E. Cancès, G. Stoltz, V.N. Staroverov, G.E. Scuseria and E.R. Davidson, Local exchange potentials for electronic structure calculations. MathematicS In Action 2 (2009) 1-42.

[11] C. Canuto, M.Y. Hussaini, A. Quarteroni and T.A. Zang, Spectral methods: fundamentals in single domains. Springer (2006).

[12] I. Catto, C. Le Bris and P.-L. Lions, Mathematical theory of thermodynamic limits: Thomas-Fermi type models. Oxford University Press (1998).

[13] H. Chen, X. Gong, L. He and A. Zhou, Convergence of adaptive finite element approximations for nonlinear eigenvalue problems. arXiv preprint, http://arxiv.org/pdf/1001.2344.

[14] H. Chen, X. Gong and A. Zhou, Numerical approximations of a nonlinear eigenvalue problem and applications to a density functional model. Math. Methods Appl. Sci. 33 (2010) 1723-1742.

[15] R.M. Dreizler and E.K.U. Gross, Density functional theory. Springer (1990).

[16] A. Edelman, T.A. Arias and S.T. Smith, The geometry of algorithms with orthogonality constraints. SIAM J. Matrix Anal. Appl. 20 (1998) 303-353.

[17] V. Gavini, J. Knap, K. Bhattacharya and M. Ortiz, Non-periodic finite-element formulation of orbital-free density functional theory. J. Mech. Phys. Solids 55 (2007) 669-696.

[18] D. Gilbarg and N.S. Trudinger, Elliptic partial differential equations of second order, 3rd edition. Springer (1998).

[19] X. Gonze et al., ABINIT: first-principles approach to material and nanosystem properties. Computer Phys. Comm. 180 (2009) $2582-2615$.

[20] P. Hohenberg and W. Kohn, Inhomogeneous electron gas. Phys. Rev. 136 (1964) B864-B871.

[21] W. Kohn and L.J. Sham, Self-consistent equations including exchange and correlation effects. Phys. Rev. 140 (1965) A1133A1138.

[22] B. Langwallner, C. Ortner and E. Süli, Existence and convergence results for the Galerkin approximation of an electronic density functional. Math. Mod. Methods Appl. Sci. 20 (2010) 2237-2265.

[23] C. Le Bris, Ph.D. thesis, École Polytechnique (1993).

[24] W.A. Lester Jr. Ed., Recent advances in Quantum Monte Carlo methods. World Sientific (1997).

[25] W.A. Lester Jr., S.M. Rothstein and S. Tanaka Eds., Recent advances in Quantum Monte Carlo methods, Part II, World Sientific (2002).

[26] M. Levy, Universal variational functionals of electron densities, first order density matrices, and natural spin-orbitals and solution of the V-representability problem. Proc. Natl. Acad. Sci. U.S.A. 76 (1979) 6062-6065.

[27] E.H. Lieb, Thomas-Fermi and related theories of atoms and molecules. Rev. Mod. Phys. 53 (1981) 603-641.

[28] E.H. Lieb, Density Functional for Coulomb systems. Int. J. Quant. Chem. 24 (1983) 243-277.

[29] Y. Maday and G. Turinici, Error bars and quadratically convergent methods for the numerical simulation of the Hartree-Fock equations. Numer. Math. 94 (2003) 739-770.

[30] W. Sickel, Superposition of functions in Sobolev spaces of fractional order. A survey. Banach Center Publ. 27 (1992) $481-497$.

[31] P. Suryanarayana, V. Gavini, T. Blesgen, K. Bhattacharya and M. Ortiz, Non-periodic finite-element formulation of KohnSham density functional theory. J. Mech. Phys. Solids 58 (2010) 256-280.

[32] N. Troullier and J.L. Martins, A straightforward method for generating soft transferable pseudopotentials. Solid State Commun. 74 (1990) 613-616.

[33] S. Valone, Consequences of extending 1matrix energy functionals from purestate representable to all ensemble representable 1 matrices. J. Chem. Phys. 73 (1980) 1344-1349.

[34] Y.A. Wang and E.A. Carter, Orbital-free kinetic energy density functional theory, in Theoretical methods in condensed phase chemistry, Progress in theoretical chemistry and physics 5. Kluwer (2000) 117-184.

[35] A. Zhou, Finite dimensional approximations for the electronic ground state solution of a molecular system. Math. Methods Appl. Sci. 30 (2007) 429-447. 